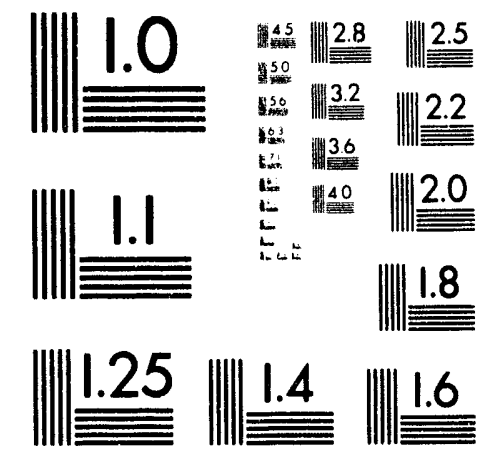



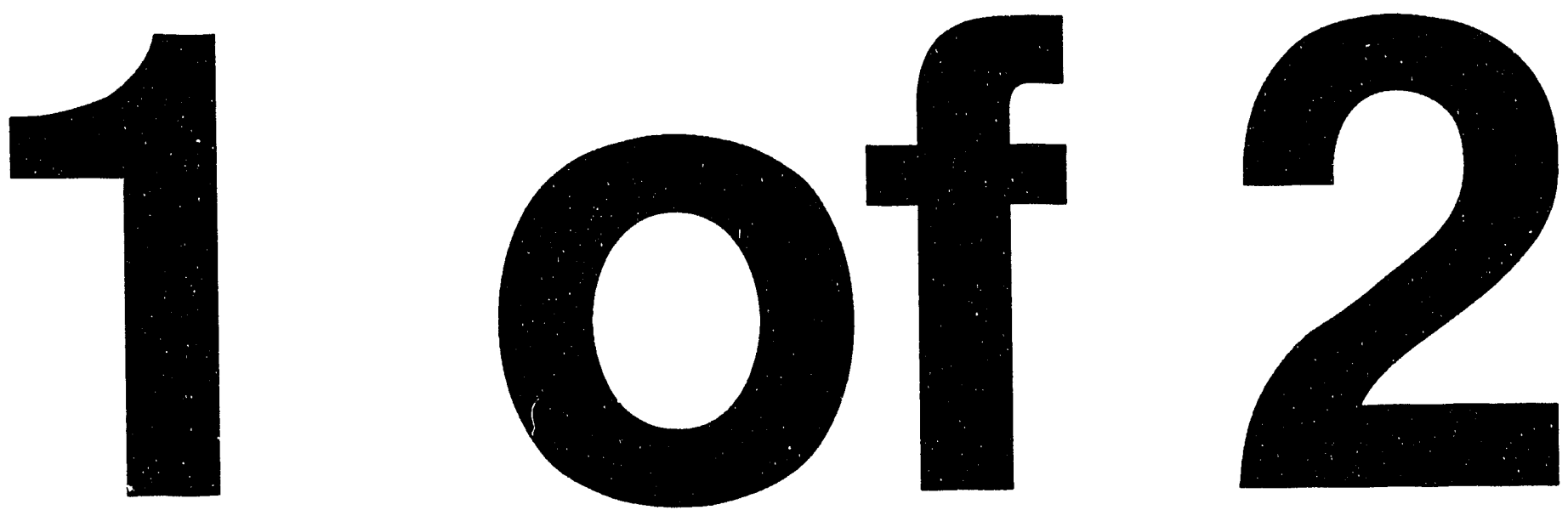


\section{Radionuclide Air Emissions Report for the Hanford Site Calendar Year 1992}

L. P. Diediker

A. R. Johnson

Westinghouse Hanford Company

K. Rhoads

D. L. Klages

J. K. Soldat

Pacific Northwest Laboratory

D. J. Rokkan

Science Applications International Corporation

Date Published

June 1993

Prepared for the U.S. Department of Energy

Office of Environmental Restoration and

Waste Management

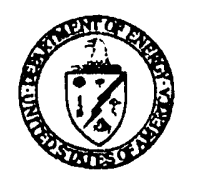

United States

Department of Energy

PO Box 550

Richland, Washington 99352 


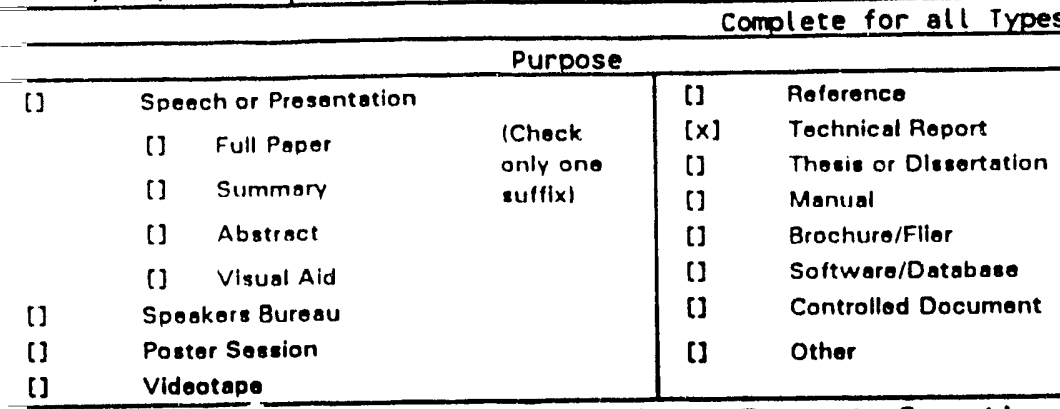

Title Radionuclide Air Emissions Report for the Hanford Site, Calendar Year 1992

New or novel (patentablel subject mat:-r? [X] No [] Yoo If "Yos", has disclosuro been submitted by WHC or other company? [] No [] Yos Disclosure Nolel.

Copyrights? [X] No [] Yoo

"Yes", has written permission been oranted?

[] No [] Yoe (Attach Pormiesion)

ID Number (include revision, volume, etc.)
$D O E / R L-93-36$

List at tachments.

Date Release Required

$6 / 18 / 93$

\begin{tabular}{|c|c|c|c|c|}
\hline Title of Conference or Meeting & & Group or Society Sponsoring & & \\
\hline Date(s) of Conference or Meeting & City/state & $\begin{array}{l}\text { Will proceedings be published? } \\
\text { Will material be handed out? }\end{array}$ & $\begin{array}{l}\text { Yes } \\
\text { Yes }\end{array}$ & $\begin{array}{l}\text { No } \\
\text { No }\end{array}$ \\
\hline
\end{tabular}

Title of Journal

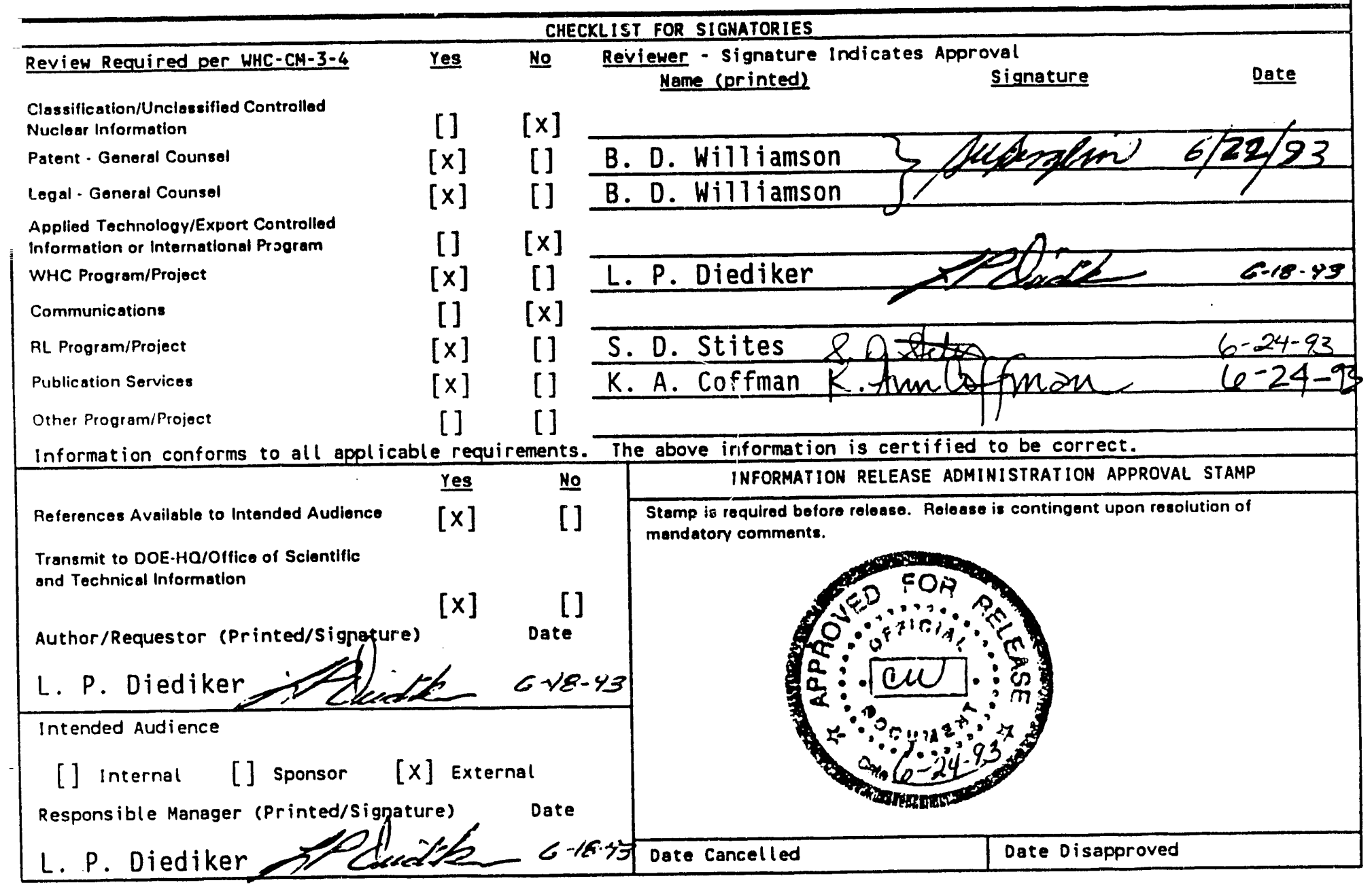




\title{
RADIONUCLIDE AIR EMISSIONS REPORT FOR THE HANFORD SITE, CALENDAR YEAR 1992
}

\begin{abstract}
This report documents radionuclide air emissions from the Hanford Site in 1992 and the resulting effective dose equivalent to any member of the public. The report has been prepared and will be submitted in accordance with reporting requirements in the Code of Federal Regulations, Title 40, Protection of the Environment, Part 61, "National Emissions Standards for Hazardous Air Pollutants, " Subpart H, "National Emission Standards for Emissions of Radionuclides Other than Radon from Department of Energy Facilities."
\end{abstract}

Mr. Steven D. Stites, of the U.S. Department of Energy, Richland Field Office, may be contacted about the information in this document. 
DOE/RL-93-36

This page intentionally left blank. 


\section{CONTENTS}

1.0 FACILITY INFORMATION $\ldots \ldots \ldots \ldots \ldots \ldots \ldots \ldots \ldots \ldots \ldots \ldots \ldots \ldots \ldots .11$

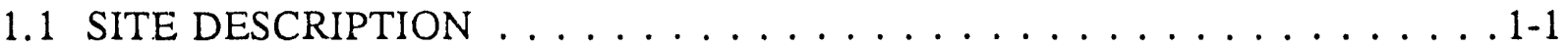

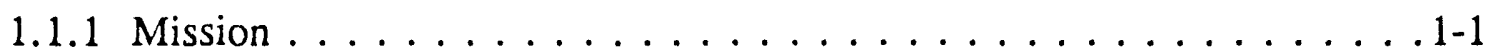

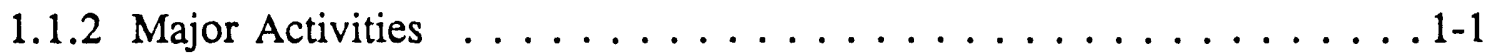

1.1.3 Site Characteristics . . . . . . . . . . . . . . 1-4

1.2 POINT-SOURCE DESCRIPTIONS $\ldots \ldots \ldots \ldots \ldots \ldots \ldots \ldots \ldots$

1.2.1 Radionuclide Air Emissions from Point Sources . . . . . . . . 1-5

1.2 .2100 Areas Facilities . . . . . . . . . . . . . . . 1-6

1.2.3 200 Areas Facilities . . . . . . . . . . . . . . . . . 1-9

1.2 .4300 Area Facilities . . . . . . . . . . . . . . . . 1-20

1.2 .5400 Area Facilities . . . . . . . . . . . . . . . . . 1-26

1.2 .6600 Area Facilities . . . . . . . . . . . . . . . . . . . 1-28

2.0 RADIONUCLIDE AIR EMISSIONS DATA ON POINT SOURCES . . . . . . . 2-1

3.0 POINT-SOURCE EMISSION DOSE ASSESSMENTS $\ldots \ldots \ldots \ldots \ldots \ldots$. . . . . .

3.1 DESCRIPTION OF DOSE MODEL . . . . . . . . . . . 3-1

3.2 SUMMARY OF INPUT PARAMETERS $\ldots \ldots \ldots \ldots \ldots \ldots \ldots \ldots$. $\ldots \ldots \ldots$

3.3 COMPLIANCE ASSESSMENT $\ldots \ldots \ldots \ldots \ldots \ldots \ldots \ldots \ldots \ldots$

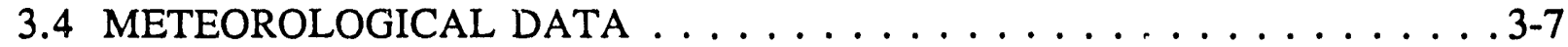

3.5 UNPLANNED RELEASES OF RADIONUCLIDES

TO THE ATMOSPHERE $\ldots \ldots \ldots \ldots \ldots \ldots \ldots \ldots \ldots \ldots \ldots$

3.6 ADDITIONAL INFORMATION $\ldots \ldots \ldots \ldots \ldots \ldots \ldots \ldots \ldots .7$

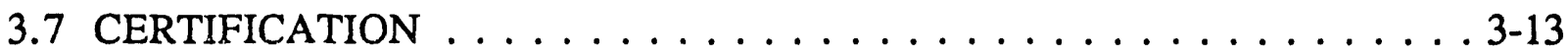

4.0 DIFFUSE-SOURCE EMISSIONS . . . . . . . . . . . . . . 4-1

4.1 DIFFUSE EMISSIONS FROM UNMONITORED SOURCES $\ldots \ldots \ldots \ldots$. . .

4.2 ESTIMATED RADIONUCLIDE AIR EMISSION RELEASES AND RESULTING DOSE TO ANY MEMBER OF THE PUBLIC

FROM DIFFUSE AND UNMONITORED SOURCES . . . . . . . 4-3

4.2.1 Methods for Assessing Diffuse-Source Emissions . . . . . . . . . 4-3

4.2.2 Results of Diffuse-Source Evaluation . . . . . . . . . . . . . . . 4-4

4.2.3 Estimate of Uncertainty in Dose from Diffuse-Emission Sources . . . 4-7

4.2.4 Discussion . . . . . . . . . . . . . . . . . . . 4-8

4.3 DIFFUSE-EMISSION SOURCES . . . . . . . . . . . . . . . . . . 4-9

4.3.1 Description of Diffuse-Emission Sources . . . . . . . . 4-10

5.0 SUPPLEMENTAL INFORMATION $\ldots \ldots \ldots \ldots \ldots \ldots \ldots \ldots$.1

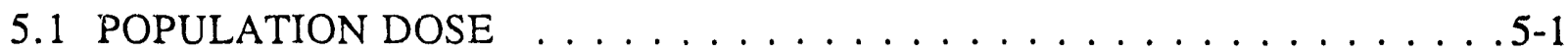

5.2 COMPLIANCE STATUS WITH SUBPARTS Q AND T

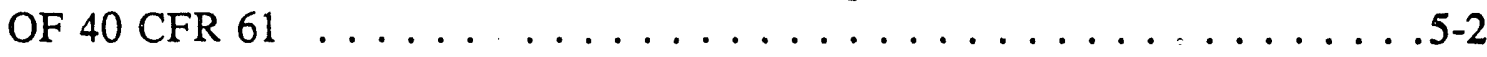




\section{CONTENTS (continued)}

5.3 EM!.jSION OF RADON-220 FROM SOURCES CONTAINING

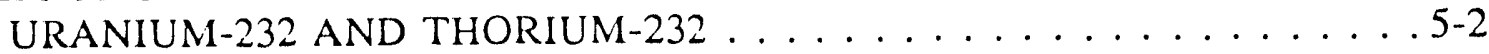

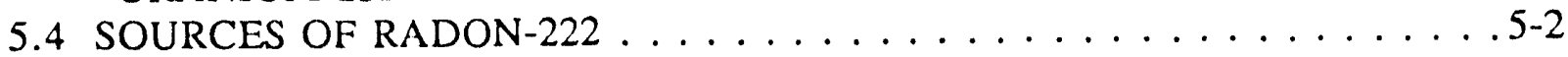

5.5 EMISSION POINTS SUBJECT TO MONITORING REQUIREMENTS

CFR 61.93(b) AND THE COSTS TO UPGRADE NONCOMPLIANT MONITORING SYSTEMS $\ldots \ldots \ldots \ldots \ldots \ldots$ 5-3

5.6 PERIODIC CONFIRMATORY MEASUREMENT PLANS . . . . . . . . 5 5-4,

5.7 STATUS OF COMPLIANCE WITH QA PROGRAM CRITERIA DESCRIBED IN APPENDIX B, METHOD 114,40 CFR $61 \ldots \ldots . .5$ 5-4

6.0 REFERENCES . . . . . . . . . . . . . . . . . 6-1 
DOE/RL-93-36

\section{LIST OF FIGURES}

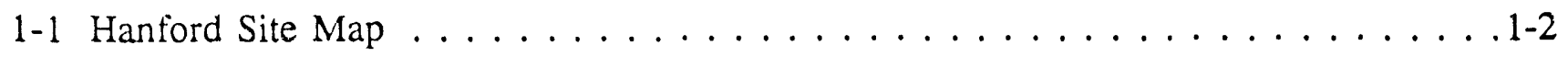

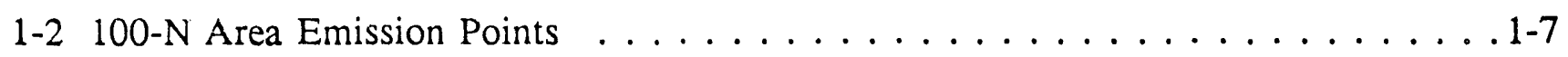

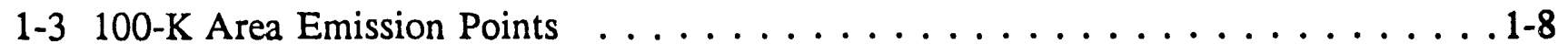

1-4 200 East Area Emission Points . . . . . . . . . . . . . . . 1-11

1-5 200 West Area Emission Points . . . . . . . . . . . . . . . 1-12

1-6 300 Area Emission Points . . . . . . . . . . . . . . . . . 1-21

1-7 400 Area Emission Points . . . . . . . . . . . . . . . . . . 1-27

1-8 600 Area Emission Points . . . . . . . . . . . . . . . . . . . . 1-29

4-1 Monthly Average Gross Beta Radioactivity in Airborne

Particulate Samples, 1981 through $1991 \ldots \ldots$. . . . . . . . . . . . 4-2 


\section{LIST OF TABLES}

2-1 Hanford Site Radionuclide Air Emissions Data for 1992 . . . . . . . . . . . . 2-2

2-2 Distances to the Nearest Offsite Residences from

Hanford Site Areas . . . . . . . . . . . . . . . . . . . 2-12

3-1 Release Estimates of Hanford Site

Radionuclide Air Emissions in $1992 \ldots \ldots$. . . . . . . . . . . . . . . . 3-2

3-2 CAP-88 Unit Dose Factors for the Offsite Hypothetical

Maximally Exposed Individual Affected by Radionuclide

Air Emissions from the Hanford Site in $1992 \ldots \ldots$. . . . . . . . . . . . 3-3

3-3 CAP-88 Dose Estimates for the Offsite Hypothetical

Maximally Exposed Individual Affected by Radionuclide

Air Emissions from the Hanford Site in $1992 \ldots \ldots \ldots$. . . . . . . . . . . . . . . .

3-4 Total Doses from Hanford Site Radionuclide

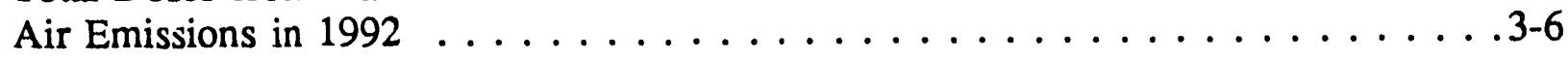

3-5 Annual Average Dispersion Around the 100-N Area

in 1992 for an 89 -Meter Release Height $\ldots \ldots \ldots \ldots \ldots$. . . . . . . . . . . . . .

3-6 Annual Average Dispersion Around the 200 Areas

in 1992 for an $89-$ Meter Release Height . . . . . . . . . . . . . . . . 3-9

3-7 Annual Average Dispersion Around the 300 Area

in 1992 for a $10-$ Meter Release Height . . . . . . . . . . . . . . 3-10

3-8 Annual Average Dispersion Around the 300 Area

During 1992 for a 61 -Meter Release Height . . . . . . . . . . . . . . 3-11

3-9 Annual Average Dispersion Around the 400 Area

During 1992 for a 10-Meter Release Height . . . . . . . . . . . . . . . 3-12

4-1 Estimated 1991 Hanford Site Diffuse Emissions and Resulting Dose . . . . . . 4-5

4-2 Types of Waste and Number of Waste Sites at the Hanford Site . . . . . . . . 4-11

4-3 Types of Diffuse-Emission Sources . . . . . . . . . . . . . . . 4-11

4-4 Hanford Site Surface and Underground Contamination . . . . . . . . . . . . 4-14 


\section{LIST OF TERMS}

\begin{tabular}{|c|c|}
\hline AMU & aqueous make-up \\
\hline CAM & continuous air monitor \\
\hline CFR & Code of Federal Regulations \\
\hline CWC & Central Waste Complex \\
\hline DCRT & double-contained receiver tanks \\
\hline DOE & U.S. Department of Energy \\
\hline DST & double-shell tank \\
\hline EDE & effective dose equivalent \\
\hline $\mathrm{EP}$ & emission point \\
\hline EPA & U.S. Environmental Protection Agency \\
\hline ESP & emission sampling point \\
\hline FFTF & Fast Flux Test Facility \\
\hline GTF & Grout Treatment Facility \\
\hline HEPA & high-efficiency particulate air (filter) \\
\hline $\mathrm{HT}$ & elemental tritium \\
\hline HTO & tritiated water vapor \\
\hline HVAC & heating, ventilation, and air conditioning \\
\hline MASF & Maintenance and Storage Facility \\
\hline MEI & maximally exposed individual (hypothetical) \\
\hline MW & Mixed Waste \\
\hline NCAW & neutralized current acid waste \\
\hline $\mathrm{P} \& \mathrm{O}$ & pipe and operating \\
\hline PEDF & Protective Equipment Decontamination Facility \\
\hline PFP & Plutonium Finishing Plant \\
\hline PNL & Pacific Northwest Laboratory \\
\hline PR & product removal \\
\hline PRF & Plutonium Reclamation Facility \\
\hline PSTF & Purgewater Storage and Treatment Facility \\
\hline PUREX & Plutonium-Uranium Extraction (Plant) \\
\hline PWR & pressurized water (cooled) reactor \\
\hline REDOX & Reduction-Oxidation (Plant) \\
\hline $\mathrm{RL}$ & U.S. Department of Energy, Richland Operations Office \\
\hline SNM & special nuclear materials \\
\hline SST & single-shell tank \\
\hline Supply System & Washington Public Power Supply System \\
\hline TRIGA & Test Reactor and Isotope Production by General Atomics \\
\hline TRU & transuranic \\
\hline TRUSAF & Transuranic Waste Storage anc Assay Facility \\
\hline $\mathrm{UO}_{3}$ Plant & Uranium-Oxide Plant \\
\hline WAC & Waste Acceptance Criteria \\
\hline WESF & Waste Encapsulation Storage Facility \\
\hline Westinghouse Hanford & Westinghouse Hanford Company \\
\hline
\end{tabular}


DOE/RL-93-36

This page intentionally left blank. 


\subsection{FACILITY INFORMATION}

\subsection{SITE DESCRIPTION}

\subsubsection{Mission}

The federal government acquired the Hanford Site in 1943 to construct and operate facilities to produce plutonium for the atomic weapons program during World War II. For more than 30 years, Hanford Site facilities were dedicated primarily to producing plutonium for national defense and managing the wastes generated by chemical processing operations. In more recent years, defense programs have declined considerably while new programs have emerged involving research and development of waste disposal technologies, renewable energy technologies, and, in particular, cleanup of contamination from past operational practices.

The current Hanford Site mission includes the following activities:

- Environmental Restoration: Restoring approximately 1,500 inactive waste sites and either decontaminating or demolishing about 100 surplus facilities

- Tank Waste Remediation: Managing the following waste types: high-level and low-level, hazardous, mixed, transuranic (TRU), TRU mixed, and sanitary

- Research and Development: Research and development in basic sciences, health and environmental sciences, and magnetic fusion

- Defense Production: Activities related to defense production have decreased significantly. $\mathrm{N}$ Reactor is now in transition to permanent shutdown. The Plutonium-Uranium Extraction (PUREX) Plant is in transition to a shutdown mode while approval for deactivation is being pursued. The Plutonium Finishing Plant (PFP), now set up to process plutonium scrap, did not operate in 1992. The 300 Area fuel fabrication facilities, where the uranium fuel elements for $\mathrm{N}$ Reactor were made, are shutdown.

\subsubsection{Major Activities}

Four major U.S. Department of Energy (DOE) operating areas exist at the Hanford Site: the 110, 200, 300, and 400 Areas (Figure 1-1). The 100 Areas include the $\mathbf{N}$ Reactor and eight deactivated production reactors, with their support facilities, all located near the Columbia River. The 200 Areas, a plateau about $7.0 \mathrm{mi}(11.3 \mathrm{~km})$ from the river, have the PUREX Plant, PFP, Uranium-Oxide $\left(\mathrm{UO}_{3}\right)$ Plant, and waste management facilities. The 300 Area, just north of the city of Richland, contains research and development 
Figure 1-1. Hanford Site Map.

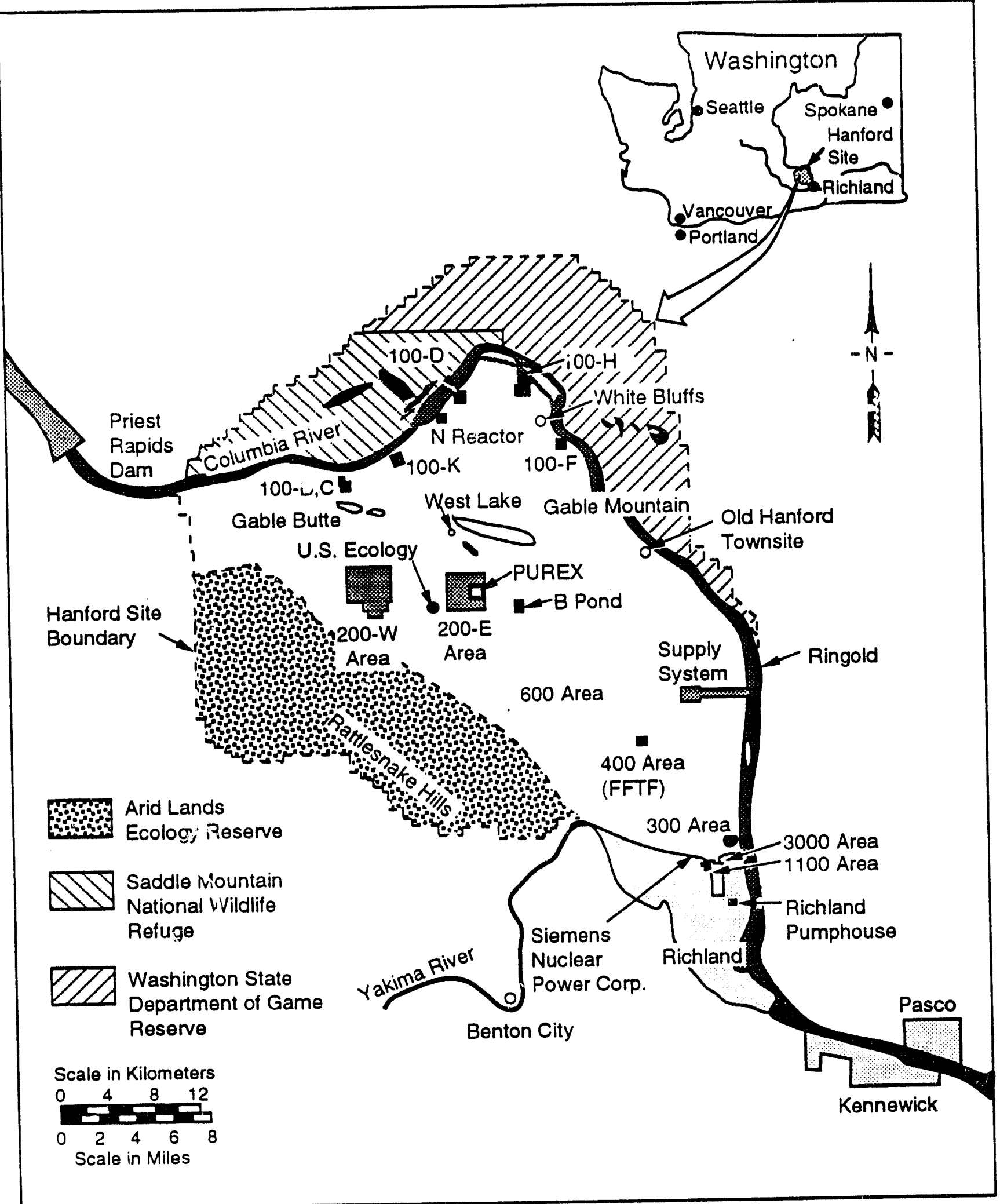


laboratories and the shutdown $\mathbf{N}$ Reactor fuel fabrication facilities. The 400 Area is the location of the Fast Fli.: Test Facility (FFTF), approximately $5.5 \mathrm{mi}(8.8 \mathrm{~km})$ northwest of the 300 Area.

The operating history of major facilities for 1992 is summarized below.

- PFP did not operate.

- The PUREX Plant did not operate in 1992, being in standby mode.

- The Grout Treatment Facility (GTF) remained in standby mode through the year.

- B Plant is continuing its revised primary mission of safely managing the radioactive inventories stored in its facilities.

- The N Reactor has not operated since January 1987 and is now in transition to permanent shutdown. All nuclear fuel was removed from the reactor core and the major piping systems drained.

- $\quad$ FFTF operaiions were placed in a "hot" standby condition, pending Congressional authorization to fund operations and a determination for a new mission, as directed by DOE.

The DOE contractors and their management responsbilities for facilities at the Hanford Site that have radionuclide air emissions are identified below.

- Westinghouse Hanford Company (Westinghouse Hanford). Manage the 200 Areas tank farms containing high-level waste; oversee environmental restoration activities; conduct liquid effluent and air emissions monitoring at DOE facilities it manages and near-facility environmental surveillance; manage the FFTF test reactor, the transition of N Reactor to permanent shutdown, and N Reactor irradiated fuel stored in the two 100-K Area water-filled storage basins; decommission old facilities; and provide Site support services, such as fire protection, central stores, and the generation and distribution of electrical power.

- Battelle Memorial Institute. Manage the Pacific Northwest Laboratory (PNL) for DOE, including research and development in the physical, chemical, life, and environmental sciences; produce advanced methods of nuclear waste management; and conduct environmental monitoring on and off the Hanford Site and liquid effluent and air emission monitoring at the DOE facilities it manages. 
Privately owned facilities located at the Hanford Site include: (1) the Washington Public Power Supply System (Supply System) Hanford Generating Project, adjacent to N Reactor; (2) a low-level radioactive-waste burial site operated by U.S. Ecology, Richland, Washington, on the 200 Area plateau; and (3) the Supply System power reactor (WNP-2) and office buildings, located near the Columbia River, several kilometers north of the 300 Area and east of the 400 Area. The Siemens Nuclear Power Corporation fuel fabrication facility is immediately adjacent to the southern boundary of the Hanforc Site. Emissions from these privately owned facilities are not included in this report.

\subsubsection{Site Characteristics}

The Hanford Site (Figure 1-1) is located in a rural region of southeastern Washington state and occupies an area of about $560 \mathrm{mi}^{2}\left(1,450 \mathrm{~km}^{2}\right)$. It lies about $200 \mathrm{mi}(320 \mathrm{~km})$ northeast of Portland, Oregon; $170 \mathrm{mi}(270 \mathrm{~km})$ southeast of Seattle, Washington; and $124 \mathrm{mi}(200 \mathrm{~km})$ southwest of Spokane, Washington.

1.1.3.1 Natural Characteristics. The semiarid land of the Hanford Site has a sparse covering of desert shrubs and drought-resistant grasses. Sagebrush, cheatgrass, and bluegrass are the most broadly distributed types of vegetation. The Great Basin pockei mouse is th most abundant mammal. The most populous big-game animal is the mule deer and among small-game animals, the cottontail rabbit. Coyotes also are quite plentiful. In the winter, bald eagles regularly visit areas along the Columbia River.

The Columbia River, originating in the mountains of eastern British Columbia, Canara, flows through the northern reach of the Hanford Site. Farther downstream the river also forms part of the eastern boundary of the Hanford Site. The river drains a total area of approximately $27,300 \mathrm{mi}^{2}\left(70,800 \mathrm{~km}^{2}\right)$ en route to the Pacific Ocean. The Columbia River flow is regulated by 11 dams within the United States, 7 upstream and 4 downstream of the Hanford Site. Other surface water on the Hanford Site consists of West Lake (a small natural pond), Rattlesnake Springs, Dry Creek, and a number of ditches and artificial ponds created for routine disposal of waste water.

The climate is dry and mild, and the area receives approximately $16 \mathrm{~cm}(6.3 \mathrm{in.})$ of precipitation annually. About $40 \%$ of the total precipitation occurs during November, December, and January; only $10 \%$ falls in July, August, and September. Approximately $45 \%$ of all precipitation from December through February is snow. The average minimum and maximum temperatures in July are $61^{\circ} \mathrm{F}\left(16^{\circ} \mathrm{C}\right)$ and $90^{\circ} \mathrm{F}\left(32^{\circ} \mathrm{C}\right)$, respectively. For January the average minimum and maximum temperatures are $21^{\circ} \mathrm{F}\left(-6^{\circ} \mathrm{C}\right)$ and $37^{\circ} \mathrm{F}$ $\left(3^{\circ} \mathrm{C}\right)$, respectively.

Monthly average $50-\mathrm{ft}(15-\mathrm{m})$ wind speeds range from about $9.3 \mathrm{mph}(15 \mathrm{~km} / \mathrm{h})$ in summer to $6.1 \mathrm{mph}(10 \mathrm{~km} / \mathrm{h})$ in winter. The prevailing winds are from the northwest, with occasional strong gusts. During the evening, the surface winds are influenced by cold-air 
drainage into the valleys. The region is a typical desert basin, in which frequent strong temperature inversions occur at night. These inversions generally deteriorate during the morning leading to unstable atmospheric conditions in mid-morning and afternoon.

Land surrounding the Hanford Site is used primarily for agriculture and livestock grazing. Agricultural lands are north and east of the Columbia River and south of the Yakima River. These areas contain orchards, vineyards, and fields of alfalfa, wheat, and vegetables. The area of the Hanford Site north of the Columbia River contains a state wildlife management area and a federal wildlife refuge. The northeast slope of Rattlesnake Hills along the southwestern boundary of the Hanford Site is designated as the Arid Lands Ecology reserve, used for ecological research by DOE. The Hanford Site also is designated as a National Environmental Research Park.

More detail on Hanford Site characteristics and activities is available in the Final Environmental Impact Statement - Disposal of Hanford Defense High-Level, Transuranic, and Tank Wastes (DOE 1987).

1.1.3.2 Demography. The most recent data on the population surrounding the Hanford Site are from the 1990 census by the Washington State Office of Financial Management. The population in the area surrounding the Hanford Site is rural, with the exception of the cities of Kennewick, Pasco, and Richland, Washington, which are near the southeast boundary of the Hanford Site. The 200 Areas meteorological tower, located approximately in the center of the Hanford Site, is a reference point often used. The total population out to a distance of $50 \mathrm{rin}(80 \mathrm{~km})$ from the meteorological tower was approximately 376,000 .

\subsection{POINT-SOURCE DESCRIPTIONS}

\subsubsection{Radionuclide Air Emissions from Point Sources}

Radionuclide air emissions from point sources generally are discharged from stacks and vents (further reference to stacks implies vents as well unless used as the proper name or description of an emission point). Sizes, shapes, and discharge paths of stacks vary because of facility requirements at the time of construction. Stack discharge heights range from a few feet to $200 \mathrm{ft}(61 \mathrm{~m})$, and flow rates range from less than $100 \mathrm{ft}^{3} / \mathrm{min}(2,832 \mathrm{~L} / \mathrm{min})$ to more than $200,000 \mathrm{ft}^{3} / \mathrm{min}(5.7$ million $\mathrm{L} / \mathrm{min})$. Stacks vary in design from horizontal to vertical, rectangular to cylindrical, and permanent to portable. Height and flow characteristics influence emission dispersion the most.

This report lists 132 stacks on the Hanford Site that in 1992: (1) normally had radionuclide emissions, (2) potentially had radionuclide emissions, or (3) could be categorized as either (1) or (2) but did not operate or was deactivated. Stack data are reported in this document when these criteria were met during 1992: (1) the stack was registered with the Washington State Department of Health (WDOH) or (2) radionuclide 
emissions from the stack were measured or had the potential of being measured. For 1992, 127 of the reported 132 stacks were registered with WDOH.

1.2.1.1 Filtration. The following methods are used singly or in combination to remove radionuclides from the emissions of most stacks: (1) high-efficiency particulate air (HEPA) filters, (2) sand filters, (3) deep-bed fiberglass filters, (4) fiberglass prefilters, (5) charcoal absorbers, and (6) water scrubbers. Generally, between one and three stages of HEPA filtration are used as the final particle removal method before an emission is released to the atmosphere.

\subsubsection{Areas Facilities}

The 100 Areas contain eight inactive production reactors, the $\mathrm{N}$ Reactor (now in transition to permanent shutdown), and associated support facilities. Radionuclide air emission points at facilities in the 100-N Area and 100-K Area are described briefly below and shown respectively in Figures 1-2 and 1-3.

1.2.2.1 N Reactor. This dual-purpose reactor operated until 1987, producing electrical power for the regional energy system and plutonium for weapons production. Because further operation of the $\mathrm{N}$ Reactor is not anticipated, the reactor and associated facilities now are in a preparatory state for full shutdown.

- 116-N. This stack exhausts filtered air from the 105-N Reactor Building. Emission monitoring consists of a record sampler.

- 109-N-Z-I. This vent did not operate in 1992. When it did, unfiltered air was released through it from the 109-N Steam Generator Cells 1 through 5. Emission monitoring consisted of a record sampler and a gross-gamma-energy monitor.

- 109-N-C-6. This vent did not operate in 1992. When it did, unfiltered air was released through it from Steam Generator Cell 6 in the 109-N Building. Emission monitoring consisted of a record sampler and a gross-gamma-energy monitor.

- 107-N. This vent exhausts filtered air from the 107-N Basin Recirculation Building. Emission monitoring consists of a record sampler. This emission point is not registered with WDOH.

- 105-N Basin Transfer Area. This vent exhausts unfiltered air from the $\mathrm{N}$ Basin and the transfer area for rail-transported well cars. Emissions are continuously monitored by a record sampler. This emission point is not registered with WDOH. 
Figure 1-2. $100-\mathrm{N}$ Area Emission Points.

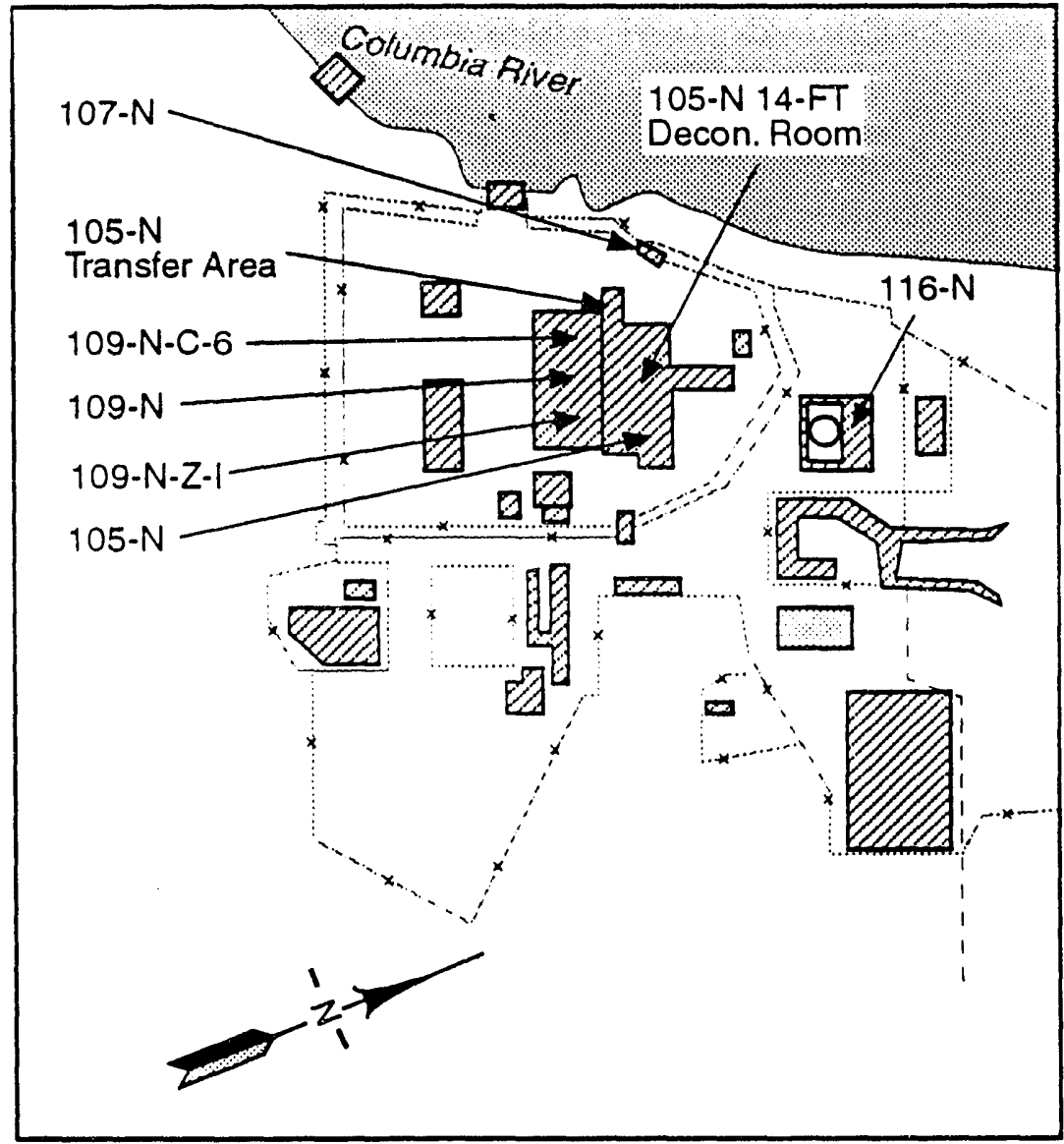

59305030.3 
Figure 1-3. 100-K Area Emission Points.

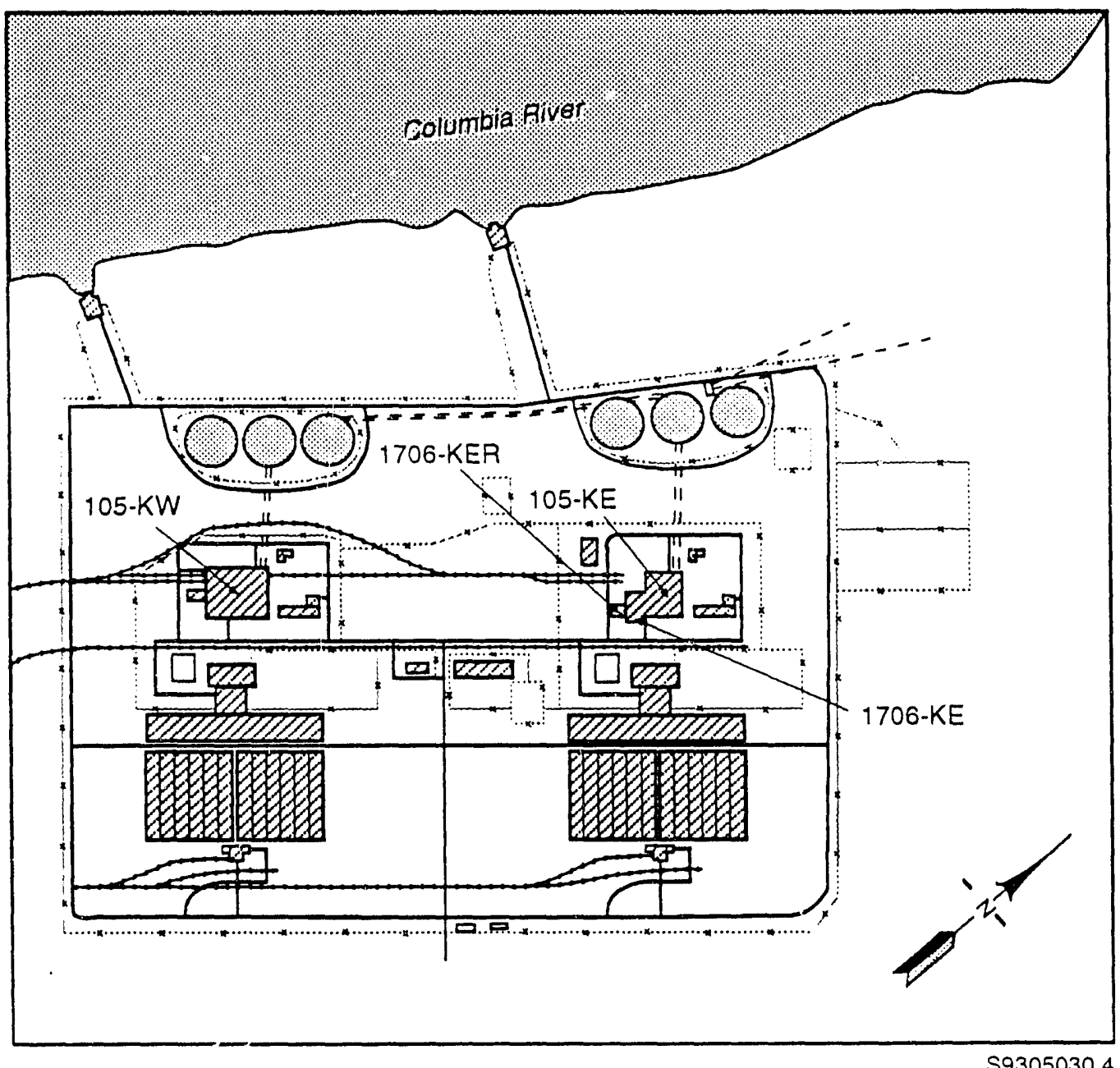


- 105-N 14-Ft. Decontamination Room. This stack exhausts filtered air from a small room in which radioactively contaminated tools are decontaminated. Ernissions are continuously monitored by a record sampler. This emission point is not registered with WDOH.

1.2.2.2 100-K East and West Areas. These areas contain two retired reactors awaiting decommissioning, two water-filled storage basins holding irradiated nuclear fuel, and radiological analysis laboratories.

- 105-KE. This vent exhausts unfiltered air from the fuel storage basin in the 105-KE Building. Emission monitoring consists of a record sampler.

- $105-\mathrm{KW}$. This vent exhausts unfiltered air from the fuel storage basin in the 105-KW Building. Emission monitoring consists of a record sampler.

- 1706-KE. This stack exhausts filtered air from the 1706-KE Laboratory. Emission monitoring consists of a record sampler.

- 1706-KER. This vent did not operate in 1992. When it did, filtered air was released through it from the basement area (approximately 27 feet below grade) of the 1706-KE Laboratory. Emission monitoring consisted of a record sampler. This emission point is not yet registered with WDOH but is expected to be within 1993.

\subsubsection{Areas Facilities}

The 200 Areas contain facilities for chemical separations, processing, and waste handling and disposal. The radionuclide air emission discharge points in the 200 East Area are shown in Figure 1-4 and those in the 200 West Area, in Figure 1-5. The PUREX Plant and related tank farm and evaporator facilities discharge volatile forms of radionuclides, specifically ${ }^{3} \mathrm{H},{ }^{106} \mathrm{Ru},{ }^{125} \mathrm{Sb}$, and ${ }^{129} \mathrm{I}$. When operating, the PUREX Plant discharged ${ }^{85} \mathrm{Kr}$, but it has not operated fully since December 1989. No activities conducted in 1992 resulted in a discharge of ${ }^{85} \mathrm{Kr}$.

1.2.3.1 PUREX Plant. The PUREX Plant is a nuclear-fuel processing plant constructed in 1956 and operated until 1972, when it was placed on standby status. Later, needed modifications were made and the PUREX Plant resumed operations in November 1983, processing a backlog of irradiated fuel from $\mathrm{N}$ Reactor. The plant was placed in standby following a stabilization run that ended in March 1990.

- 291-A-1. This stack exhausts filtered air from canyon ventilation (Cells A to $\mathrm{M})$, vessel and condenser vents, and the dissolver offgas system. Emission monitoring consists of a record sampler, an alpha continuous air monitor (CAM), and a beta-gamma CAM. 
- 296-A-1. This stack exhausts filtered air from $\mathrm{N}$ and Q Cells, Product Removal rooms, and gloveboxes in these rooms. Emission monitoring consists of a record sampler and an alpha CAM.

- 296-A-2. This stack exhausts filtered air from the west sample gallery hoods. Emission monitoring consists of a record sampler, an alpha CAM, and a beta-gamma CAM.

- 296-A-3. This stack exhausts filtered air from hoods in the east sample gallery. Emission monitoring consists of a record sampler and a beta-gamma CAM.

- 296-A-5A and 296-A-5B. These stacks exhaust filtered air from laboratory hoods. Emission monitoring for each stack consists of a record sampler, an alpha CAM, and a beta-gamma CAM.

- 296-A-6. This stack exhausts filtered room air from the east sample gallery and $U$ Cell. Emission monitoring consists of a record sampler.

- 296-A-7. This stack exhausts filtered room air from the PUREX west sample gallery and $\mathrm{R}$ Cell. Emission monitoring consists of a record sampler.

- 296-A-8. This stack exhausts filtered air from the PUREX Plant Pipe and Operating gallery and the White Room. Emission monitoring consists of a record sampler.

- 296-A-10. This stack exhausts filtered air from storage tunnel No. 2. Emission monitoring consists of a record sampler.

- 296-A-14. This stack exhausts filtered air from the 293-A Offgas Treatment and Recovery Building (for nitric acid recovery). Emission monitoring consists of a record sampler.

- 296-A-24. This stack, known as the ammonia-scrubber waste-concentrator stack, did not operate in 1992 . Its monitoring systems have been deactivated and emission points sealed. When it did operate, filtered air was released through it from the $\mathrm{E}$ and $\mathrm{F}$ Cell vessels to reduce ammonium-nitrate loading on the 291-A-1 filters. Emission monitoring consisted of a record sampler, two silver-zeolite cartridge samplers for volatile radionuclides, an alpha CAM, a beta-gamma CAM, and an ammonia monitor. 
Figure 1-4. 200 East Area Emission Points.

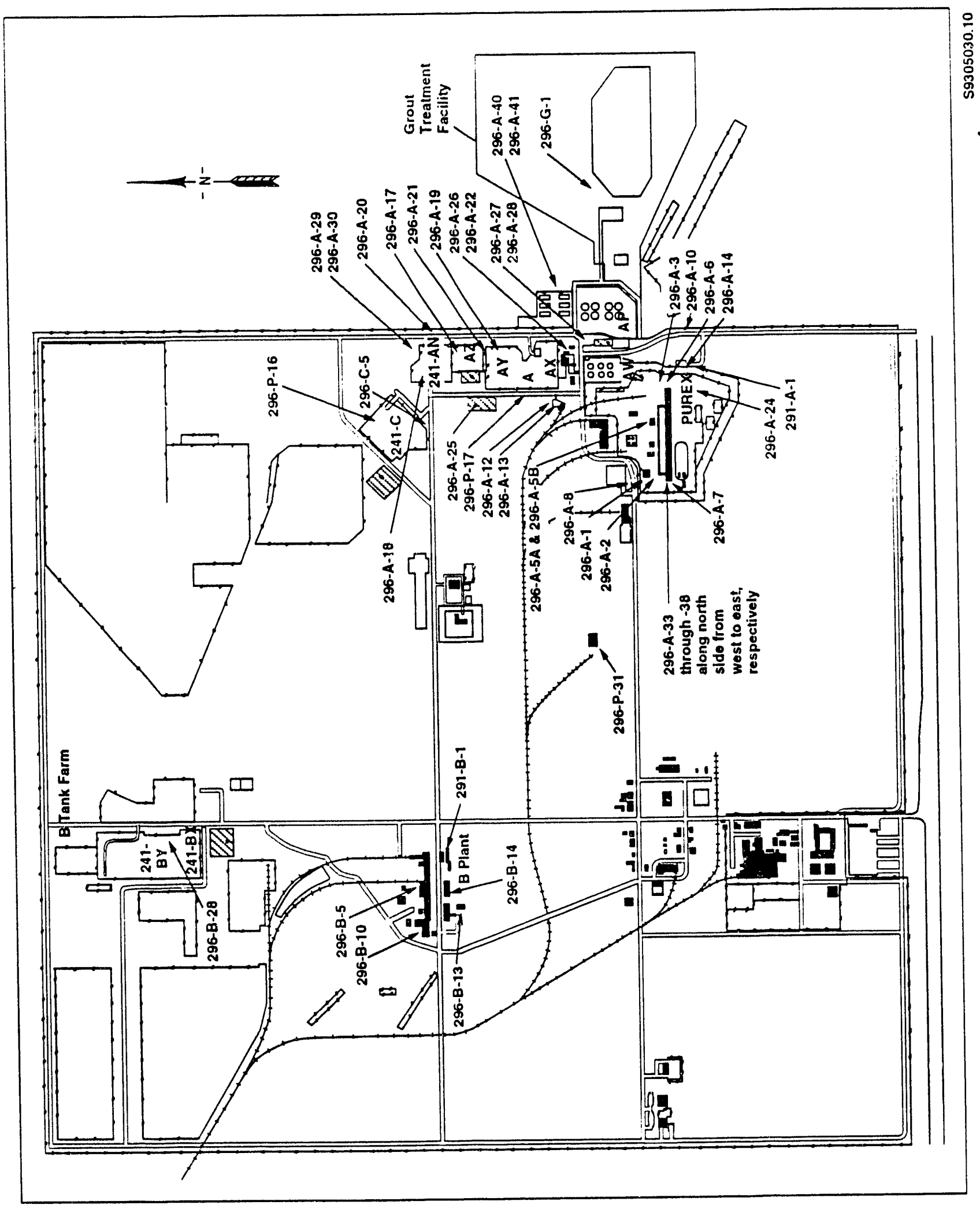


Figure 1-5. 200 West Area Emission Points.

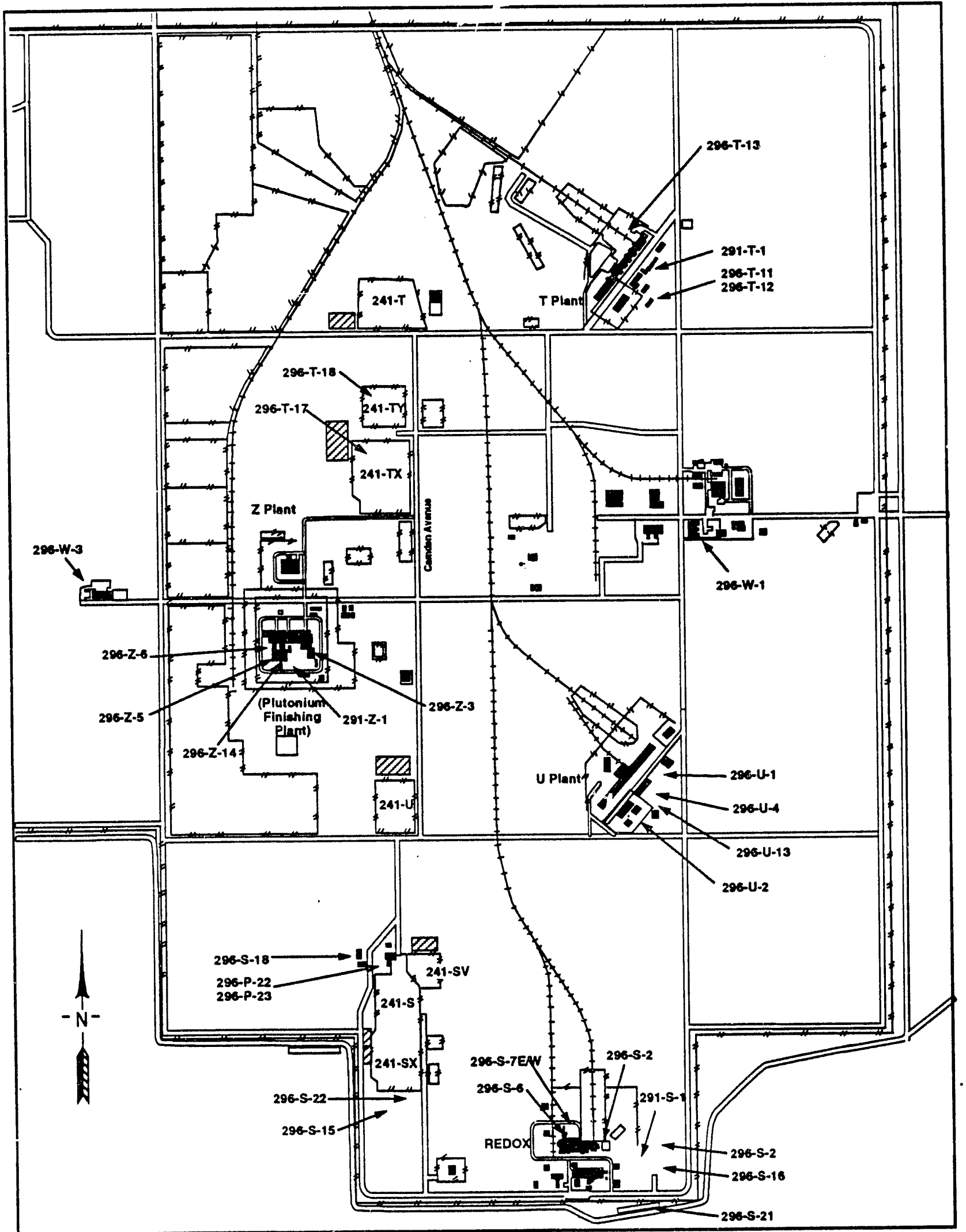


- 296-A-33, -34, -35, -36, -37, and -38. All of these exhausters are now deactivated and will be deregistered with WDOH. Exhausters 296-A-33, -35, and -37 were deactivated before 1991, and exhausters 296-A-34 and -36 by the end of 1991. Exhauster 296-A-38 operated only briefly in January 1992 before being deactivated. The exhauster emissions had been continuously monitored by record samplers and beta-gamma CAMs.

1.2.3.2 B Plant and Waste Encapsulation Storage Facility. This facility contains two major operating system areas, B Plant and the Waste Encapsulation Storage Facility (WESF). B Plant was designed and used to remove ${ }^{137} \mathrm{Cs}$ and ${ }^{90} \mathrm{Sr}$ from high-level liquid waste. At WESF, the ${ }^{137} \mathrm{Cs}$ and ${ }^{90} \mathrm{Sr}$ were converted to solid strontium fluoride and cesium chloride, doubly encapsulated, and placed in water-filled storage basins. WESF now is used to store radioactive inventories, having many radioactive strontium and cesium capsules. The 221-B Building has radioactive contamination remaining from previous production campaigns that must be properly managed.

- 291-B-1. This is the B Plant main stack, which exhausts filtered air from the $\mathrm{B}$ Plant canyon, the process vessel ventilation system number 1, and the 212-B and 224-B Buildings. Emission monitoring consists of a record sampler, a backup record sampler, and a beta-gamma CAM.

- 296-B-5. This stack exhausts filtered air from the 221-BB Building, which houses the B Plant process condensate and B Plant steam condensate receiver tanks. Emission monitoring consists of a record sampler and a beta-gamma CAM.

- 296-B-10. This stack exhausts filtered air from the 225-B Building, which is also known as WESF. Emission monitoring consists of a record sampler and a beta-gamma CAM.

- 296-B-13. This stack exhausts filtered air from the 221-BF Condensate Effluent Discharge Facility, which houses process condensate retention ventilation. Emission monitoring consists of a record sampler and a beta-gamma CAM.

- 296-B-14. This stack exhausts filtered air from the 221-B Building and the vessel ventilation system No. 2. Emission monitoring consists of a record sampler and a process sampler. This stack did not operate in 1992.

1.2.3.3 AR and CR Vaults. The $A R$ and $C R$ Vaults are retention and treatment facilities for high-level radioactive liquid wastes.

- 296-A-12. This stack exhausts filtered air from the 244-AR Vault vessel ventilation system. Emission monitoring consists of a record sampler and a beta-gamma CAM. 
- 296-A-13. This stack exhausts filtered air from the 244-AR Vault canyon and cells. Emission monitoring consists of a record sampler and a beta-gamma CAM.

- 296-C-5. This stack exhausts filtered ir from the 244-CR Vault Cell and vessel ventilation. Emission monitoring consists of a record sampler and a beta-gamma CAM.

1.2.3.4 200 East Area Tank Farms (excluding the Evaporator). Radioactive waste stored in tank farms consists of sludge and salt cake in single-shell tanks (SST) and slurry in double-shell tanks (DST).

- 296-A-17. This stack exhausts filtered, noncondensable vapors from waste storage tanks in the 241-AY and -AZ Tank Farms. Emission monitoring consists of a record sampler, two silver-zeolite cartridge samplers for volatile radionuclides, and a beta-gamma CAM.

- 296-A-18. This stack exhausts filtered air from the 241-AY-101 tank annulus. Emission monitoring consists of a record sampler. This stack has been inactive since September 1991.

- 296-A-19. This stack did not operate in 1992. When it did, filtered air was released through it from the 241-AY-102 tank annulus. Emission monitoring consisted of a record sampler.

- 296-A-20. 241-AZ Tank Annuli Exhaust. This stack exhausts filtered air from the 241-AZ-101 and -102 tank annuli. Emission monitoring consists of a record sampler.

- 296-A-25. This stack exhausts filtered air from the catch tank at the 244-A lift station. Emission monitoring consists of a record sampler and a beta-gamma CAM.

- 296-A-26. This stack exhausts filtered air from the waste unloading room and sump tank at the 204-AR tank. Emission monitoring consists of a record sampler and a beta-gamma CAM.

- 296-A-27. This stack exhausts filtered air from all 241-AW tanks. Emission monitoring consists of a record sampler, two silver-zeolite cartridge samplers for volatile radionuclides, and a beta-gamma CAM.

- 296-A-28. This stack exhausts filtered air from all tank annuli in the 241-AW tank farm. Emission monitoring consists of a record sampler and a beta-gamma CAM. 
- 296-A-29. This stack exhausts filtered air from all 241-AN tanks. Emission monitoring consists of a record sampler and a beta-gamma CAM.

- 296-A-30. This stack exhausts filtered air from all tank annuli in the 241-AN tank farm. Emission monitoring consists of a record sampler and a beta-gamma CAM.

- 296-A-40. This stack exhausts filtered air from all 241-AP tanks. Emission monitoring consists of a record sampler, two silver-zeolite cartridge samplers for volatile radionuclides, and a beta-gamma CAM.

- 296-A-41. This stack exhausts filtered air from all tank annuli in the 241-AP tank farm. Emission monitoring consists of a record sampler and a beta-gamma CAM.

- 296-B-28. This stack exhausts filtered air from the 244-BX salt well receiver tanks and annulus. Emission monitoring consists of a record sampler, a beta-gamma CAM for the tank exhaust, and a beta-gamma CAM for the annulus exhaust.

- 296-P-16. A portable exhauster emits filtered air via this stack from the 241-C-104, -105, and -106 tanks. Emission monitoring consists of a record sampler and a beta-gamma CAM.

- 296-P-17. This portable exhauster emits filtered air from the 241-A-104, -105 , and -106 tanks. Emission monitoring consists of a record sampler and a beta-gamma CAM.

1.2.3.5 200 East Area Evaporator. The evaporator is used to remove most of the water from high-level radioactive liquid waste, leaving a slurry that is pumped back to tank farms.

- 296-A-21. This stack exhausts filtered air from the 242-A EvaporatorCrystallizer Building. Emission monitoring consists of a record sampler, an alpha CAM, and a beta-gamma CAM.

- 296-A-22. This stack exhausts filtered air from the 242-A EvaporatorCrystallizer vessel-ventilation system. Emission monitoring consists of a record sampler, two silver-zeolite cartridge samplers for volatile radionuclides, an alpha CAM, and a beta-gamma CAM.

1.2.3.6 Grout Treatment Facility. This facility solidifies low-level liquid waste for disposal in underground vaults.

- 296-G-1. This stack exhausts filtered air from the GTF Air Filtration Module. Emission monitoring consists of a record sampler and a beta-gamma CAM. 
1.2.3.7 209-E Critical Mass Laboratory. The building is no longer in operation. Formerly it was used for testing critical mass configurations.

- 296-P-31 (formerly 209-E). This stack exhausts filtered building ventilation air. It operated briefly in 1992 but valid emission and operating data were not available. The exhaust is sampled for particulate alpha and beta radioactivity.

1.2.3.8 S Plant. $S$ Plant is another name for the Reduction-Oxidation Plant (REDOX), which is the 202-S building. The REDOX Plant was operated as a fuei-reprocessing facility until 1967 when it was shut down.

- 291-S-1. The REDOX main stack exhausts filtered air from the REDOX canyon, vessel ventilation, and treated dissolver offgas system. Emission monitoring consists of a record sampler and a beta-gamma CAM.

- 296-S-2. This stack exhausts filtered air from REDOX north and south sample galleries, hoods, and product removal cage. Emission monitoring coissists of a record sampler.

- 296-S-4. This stack exhausts filtered air from the REDOX decontamination room and regulated shop and unfiltered air from the regulated tool room, low-level decontamination sink, and special work permit lobby. Emission monitoring consists of a record sampler.

- 296-S-6. This stack exhausts air from the REDOX Plant, the silo gallery, an organic feed tank, and a sample elevator. Emission monitoring consists of a record sampier.

- 296-S-7W. This stack exhausts filtered air from the 233-S REDOX Product building, the REDOX plutonium-processing greenhouse, and process vessel ventilation and loadout area. Emission monitoring consists of a record sampler and an alpha CAM.

1.2.3.9 T Thant. $T$ Plant is one of the original fuel reprocessing facilities. The last fuel reprocessed there was in 1956. Now the 221-T Building is used for decontaminating and repairing equipment and the 224-T Building for storing and assaying waste suspected of containing TRU.

- 291-T-1. This stack exhausts filtered air from 2<1-T canyon and process ventilation. Emission monitoring consists of a record sampler, a beta-gamma CAM, and an alpha CAM.

- 296-T-11. This stack exhausts filtered air from the 224-T Building ventilation through the east part of the plenum. Emission monitoring consists of a record sampler. 
- 296-T-11. This stack exhausts filtered air from the 224-T Building ventilation through the east part of the plenum. Emission monitoring consists of a record sampler.

- 296-T-12. This stack exhausts filtered air from the 224-T Building ventilation through the west part of the plenum. Emission monitoring consists of a record sampler.

- 296-T-13. This stack exhausts filtered air from the 221-T Building and canyon ventilation. Emission monitoring consists of a record sampler and a beta-gamma CAM.

1.2.3.10 U Plant. U Plant was constructed as a fuel reprocessing plant but never used for that purpose. Instead it was used to recover uranium from bismuth-phosphate waste and high-level radioactive wastes from tank farms. Now, however, it is a shutdown, retired facility, having a few offices and shops still in use. Located adjacent to U Plant is the Uranium-Oxide $\left(\mathrm{UO}_{3}\right)$ Plant, which formerly produced uranium trioxide from PUREX Plant solutions. In 1972 the $\mathrm{UO}_{3}$ Plant was shut down. Later it was refurbished to process uranyl nitrate hexahydrate from the PUREX Plant and restarted in March 1984. No batch processing was conducted in 1992.

- 291-U-1. This stack exhausts filtered air from U Plant and 221-U canyon ventilation. Emission monitoring consists of a record sampler and a beta-gamma CAM.

- 296-U-2. This stack exhausts filtered air from the powder-handling system in the 224-UA Building of the $\mathrm{UO}_{3}$ Plant. Emission monitoring consists of a record sampler.

- 296-U-4. This $\mathrm{UO}_{3}$ Plant stack exhausts unfiltered air from the process offgas system (vessel vent, concentrator, and calciner) on the 224-U Building. Emission monitoring consists of a record sampler.

- 296-U-13. This $\mathrm{UO}_{3}$ Plant stack did not operate in 1992. When it did, filtered air was released through it from the powder loadout hood. Emission monitoring consisted of a record sampler and beta-gamma CAM.

1.2.3.11 Plutonium Finishing Plant. PFP was in standby during 1992. Historically, PFP, located in the 200 West Area, recovered plutonium in the form of plutonium nitrate from plutonium scrap. Plutonium metal was also produced from recovered nitrate and plutonium nitrate received from the PUREX Plant. This facility now is used to recover, stabilize, and store plutonium. Recovered plutonium nitrate and plutonium nitrate solutions received from the PUREX Plant are reduced to plutonium dioxide. The reduction process stabilizes the plutonium into the state best suited for long-term storage. The mission of PFP no longer includes the production of finished plutonium metal. 
- 291-Z-1. This stack exhausts filtered air from the 234-5Z, 236-Z, and 242-Z Buildings. Emission monitoring consists of a record sampler and an alpha CAM.

- 296-Z-3. This stack exhausts filtered air from $241-Z$ vault sump and vessel ventilation. Emission monitoring consists of a record sampler and an alpha CAM.

- 296-Z-5. This stack exhausts filtered air from 2736-ZB, the shipping and receiving building. Emission monitoring consists of a record sampler and an alpha CAM.

- 296-Z-6. This stack exhausts filtered air from the 2736-ZA Building and its plutonium storage vault ventilation system. Emission monitoring consists of a record sampler and two alpha CAMs. Each alpha CAM is located on each of the two ducts feeding into this stack.

- 296-Z-10. This stack exhausts filtered air from noncontaminated working spaces in the 231-Z Building. Record sampling was discontinued several years ago because emissions were continuously below detection limits. Because of these established conditions, WDOH will be requested to deregister this stack.

- 296-Z-11. This stack exhausts filtered air from offices in the 231-Z Building. Record sampling was discontinued several years ago because emissions were continuously below detection limits. Because of these established conditions, WDOH will be requested to deregister this stack.

- 296-Z-14. This stack exhausts filtered air from the $232-Z$ Incinerator Building. Emission monitoring consists of a record sampler and two alpha CAMs.

1.2.3.12 200 West Area Tank Farms (Excluding Evaporators). Radioactive waste stored in tank farms consists of sludge and salt cake in SSTs and liquid and slurry in DSTs.

- 296-P-22. This stack exhausts filtered air from annuli in the 241-SY-101, -102 , and -103 tanks. Emission monitoring consists of a record sampler and a beta-gamma CAM.

- 296-P-23. This stack exhausts filtered air from the 241-SY-101, -102, and -103 tanks. Emission monitoring consists of a record sampler and a beta-gamma CAM.

- 296-S-15. This stack exhausts filtered air from tanks 241-SX-107 through -112 and 241-SX-114. Emission monitoring consists of a record sampler and a beta-gamma CAM. 
- 296-S-22. This stack exhausts filtered air from the 244-S salt well receiver tank and annulus. Emission monitoring consists of a record sampler, an alpha CAM, and a beta-gamma CAM.

- 296-T-18. This stack exhausts filtered air from the 244-TX salt well receiver tank and annulus. Emission monitoring consists of a record sampler, an alpha CAM, and a beta-gamma CAM.

1.2.3.13 200 West Area Evaporators. The 242-T Evaporator-Crystallizer did not operate in 1992, being shutdown. The 242-S Evaporator-Crystallizer also did not operate in 1992, but is on standby. The evaporators are designed to remove the majority of water from radioactive liquid waste, with the resulting slurry then routed to tank farms for storage.

- 296-S-18. This stack exhausts filtered air from the 242-S EvaporatorCrystallizer Building. Emission monitoring consists of a record sampler, an alpha CAM, and a beta-gamma CAM.

- 296-T-17. This stack exhausts filtered air from the 242-T EvaporatorCrystallizer and cold-cell ventilation. Emission monitoring consists of a record sampler, an alpha CAM, and a beta-gamma CAM.

1.2.3.14 2724-W Laundry. This is the Protective Equipment and Decontamination Facility, also known as the 2724-W Laundry, located in the 200 West Area. Radioactively contaminated protective clothing and equipment used by Hanford Site contractors are laundered at this facility.

- 296-W-1. This stack exhausts air from clothes dryers and a washroom. The air is scrubbed with - water wash before exhausting through the stack. Emission monitoring consists of a record sampler.

1.2.3.15 222-S Analaytical Laboratories. The 222-S Analytical Laboratories provide chemical and radiochemical analytical support for Westinghouse Hanford, which includes analysis of environmental and effluent samples, research and development, and characterization of tank farm waste.

- 296-S-16. This stack exhausts filtered air from the 219-S Building vault and waste tanks. Emission monitoring consists of a record sampler.

- 296-S-21. This stack exhausts filtered air from 222-S Laboratory hoods, gloveboxes, hot-cells, and room ventilation system. Emission monitoring consists of a record sampler, an alpha CAM, and a beta-gamma CAM.

1.2.3.16 Waste Compactor. The facility compacts the volume of radioactive solid waste. 
- 296-W-3. This exhaust discharges filtered air from the 213-W compactor. Emission monitoring consists of a record sampler and a beta-gamma CAM.

\subsubsection{Area Facilities}

The 300 Area consists primarily of laboratories, research facilities, and a steam plant. Emission points in the 300 Area are shown in Figure 1-6.

1.2.4.1 306-E Metal Fabrication Development Building. This building has been shut down, but it once housed three separate operations. These were: (1) a large high-bay used for nonfueled test article fabrication for FFTF, (2) nondestructive, radiography examination in a series of shielded cells, and (3) a small depleted-uranium-powder laboratory used to fabricate insulator pellets for the FFTF fuel pins.

- 306-E-ULAB. This stack exhausts filtered air from the Uranium Oxide Laboratories in rooms 158 and 159 . The laboratories are currently shut down. Emission monitoring consists of a record sampler.

1.2.4.2 308 Fuels Development Laboratory. The 308 Laboratory was used for fabricating mixed-oxide fuel pins used by FFTF. The facility houses a shutdown swimming-pool-type Test Reactor and Isotope Production reactor by General Atomics (TRIGA) that was used in neutron radiography of the completed pins. The 308 Building is in transition to shutdown.

- 308-GL-EX. This exhaust discharges filtered air from gloveboxes and hoods in the 308 Building. Emission monitoring consists of a record sampler and an alpha CAM.

- 308-ET-EX. This exhaust discharges filtered air from those portions of the 308 Laboratory previously associated with etching and cleaning operations, discontinued several years ago. Emission monitoring consists of a record sampler and an alpha CAM.

- 308-TRIGA. This stack discharges filtered air from the reactor hall housing the TRIGA reactor. The reactor, however, is permanently shutdown and partially defueled. Emission monitoring consists of a record sampler and an alpha CAM.

1.2.4.3 340 Complex. The 340 Complex houses the radioactive liquid waste and solid waste handling operations for the 300 Area. The 340-A Building contains six aboveground storage tanks for radioactive liquid waste. The east side of the building has a railcar loadout facility for transferring liquid waste to be shipped to the 200 Areas. The west side is a storage area for radioactive waste. 
Figure 1-6. 300 Area Emission Points.

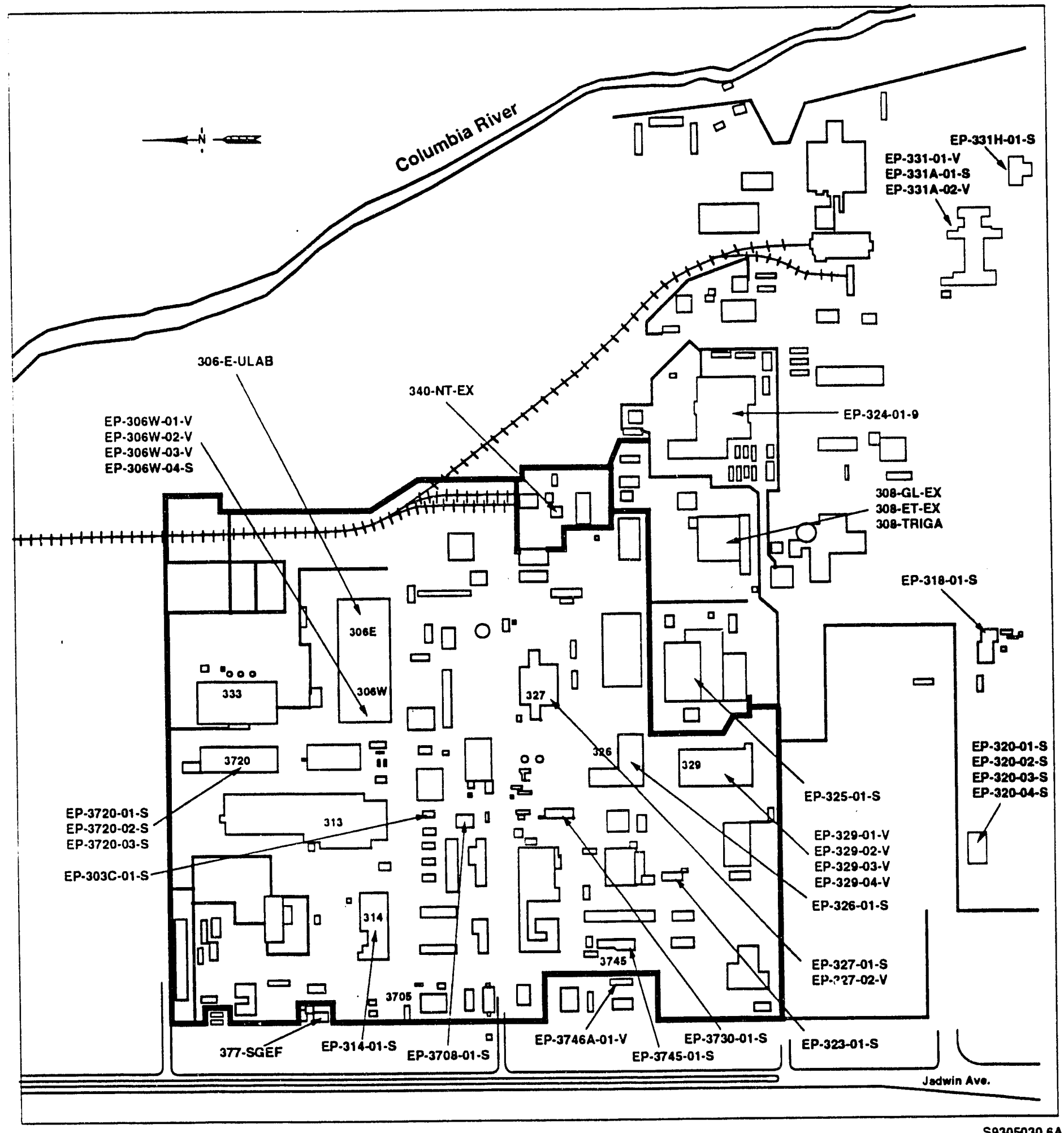


- 340-NT-EX. 340 Tank and Vault Exhaust. This stack exhausts filtered air from the 340-A Building vault and waste tanks. Emission monitoring consists of a record sampler, an alpha CAM, a beta-gamma CAM, and a radioiodine CAM.

1.2.4.4 303-C Materials Evaluation Laboratory. The building is used primarily for storage of special nuclear materials in sealed containers.

- $\quad$ Emission Point (EP)-303C-01-S. This stack exhausts filtered building ventilation air. The exhaust is sampled for particulate alpha and beta radioactivity.

1.2.4.5 306-W Materials Development Laboratory. The building contains shops and laboratory facilities for metal-working and ceramic studies.

- EP-306W-01-V. This vent exhausts filtered building ventilation air. The exhaust is sampled for particulate alpha and beta radioactivity.

- EP-306W-02-V. This vent exhausts filtered building ventilation air. The exhaust is sampled for particulate alpha and beta radioactivity.

- EP-306W-C3-V. This vent exhausts filtered building ventilation air. The exhaust is sampled for particulate alpha and beta radioactivity.

- EP-306W-04-S. This stack exhausts filtered building ventilation air from the northwest portion of the building. It is sampled for particulate alpha and beta radioactivity.

1.2.4.6 314 Engineering Development Laboratory. The building contains high-bay facilities and laboratories for engineering studies.

- EP-314-01-S. This stack exhausts filtered building ventilation air from chemistry laboratories on the north side of the building. The exhaust is sampled for particulate alpha and beta radioactivity.

1.2.4.7 318 Radiological Sciences Laboratory. The building contains facilities for calibrating radiation survey instruments and processing personnel dosimeters.

- EP-318-01-S. This stack exhausts filtered building ventilation air. The exhaust is sampled for particulate alpha and beta radioactivity.

1.2.4.8 320 Physical Sciences Laboratory. The building contains environmental radiochemistry laboratories. 
- EP-320-01-S. This stack exhausts filtered building ventilation air. The exhaust is sampled for particulate alpha and beta radioactivity.

- EP-320-02-S. This stack exhausts filtered chemistry hoods. The exhaust is sampled for particulate alpha and beta radioactivity.

- EP-320-03-S. This stack exhausts filtered chemistry hoods. The exhaust is sampled for particulate alpha and beta radioactivity.

- EP-320-04-S. This stack exhausts filtered chemistry hoods. The exhaust is sampled for particulate alpha and beta radioactivity.

1.2.4.9 323 Mechanical Properties Laboratory. The building contains facilities for investigating structural properties of irradiated materials.

- EP-323-01-S. This stack exhausts filtered building ventilation air. The exhaust is sampled for particulate alpha and beta radioactivity.

1.2.4.10 324 Waste Technology Engineering Laboratory. The building contains laboratories for performing chemical and process development activities.

- EP-324-01-S. This stack exhausts filtered building air. The exhaust is sampled for particulate alpha and beta radioactivity, ${ }^{131} \mathrm{I}$, and tritium. At present, no source of ${ }^{131} \mathrm{I}$ exists in the building.

1.2.4.11 325 Applied Chemistry Laboratory. The building contains radiochemistry laboratories.

- EP-325-01-S. This stack exhausts filtered building air. The exhaust is sampled for particulate alpha and beta radioactivity, ${ }^{131} \mathrm{I}$, and tritium. At present, no source of ${ }^{131} \mathrm{I}$ exists in the building.

1.2.4.12 326 Materials Sciences Laboratory. The building contains laboratories and equipment for studies of metallurgical, chemical, and physical behavior of reactor components and fuel materials.

- EP-326-01-S. This stack exhausts filtered building ventilation air. The exhaust is sampled for particulate alpha and beta radioactivity and tritium.

1.2.4.13 327 Post Irradiation Testing Laboratory. The building contains hot-cells for examining and testing irradiated materials.

- EP-327-01-S. This stack exhausts filtered building air. The exhaust is sampled for particulate alpha and beta radioactivity and ${ }^{131} \mathrm{I}$. At present, no source of ${ }^{131} \mathrm{I}$ exists in the building. 
- EP-327-02-V. This roof vent exhausts air from the decontamination cell. The exhaust is sampled for particulate alpha and beta radioactivity and ${ }^{131} \mathrm{I}$. At present, no source of ${ }^{131} \mathrm{I}$ exists in the building.

1.2.4.14 329 Chemical Sciences Laboratory. The building contains chemistry laboratories for radioanalytical studies, environmental radionuclide studies, and radiation detection instrumentation development.

- EP-329-01-V. This vent exhausts filtered building ventilation air. The exhaust is sampled for particulate alpha and beta radioactivity.

- EP-329-02-V. This vent exhausts filtered building ventilation air. The exhaust is sampled for particulate alpha and beta radioactivity.

- EP-329-03-V. This vent exhausts filtered building ventilation air. The exhaust is sampled for particulate alpha and beta radioactivity.

1.2.4.15 329 Neutron Multiplier Facility. The building contains a neutron multiplier source for activation analysis and on-line spectrometry of fission products.

- EP-329-04-V. This vent exhausts filtered building ventilation air. The exhaust is sampled for particulate alpha and beta radioactivity.

1.2.4.16 331 Life Sciences Laboratory. The building contains facilities for biological and ecological research studies.

- EP-331-01-V. This vent exhausts filtered building ventilation air. The exhaust had been sampled at two separate points (referred to as D1 and D2) for particulate alpha and beta radioactivity. Now only D1 is used. Sampling point D2 is registered unnecessarily with WDOH, who in 1993 will be given a request to deregister it. Proper registration of the stack will be requested, too. WDOH has already been provided a full explanation on this stack and the two associated sampling points.

- EP-331A-01-S. This stack exhausts filtered building ventilation air. The exhaust is sampled for particulate alpha and beta radioactivity.

- EP-331A-02-V. This vent exhausts filtered building ventilation air from the liquid waste transfer room. The room contains facilities for transferring and storing radioactive liquid waste used in biological research studies. The exhaust is sampled for particulate alpha and beta radioactivity.

1.2.4.17 331-H Aerosol Wind Tunnel Research Facility. The building contains a wind tunnel for atmospheric pollutant deposition studies. There are no unsealed radionuclides in use in the facility at this time. 
- EP-331H-01-S. This stack exhausts filtered building ventilation air. The exhaust is sampled for particulate alpha and beta radioactivity.

1.2.4.18 377 Steam Generator Facility. The facility was formerly used for studying a steam generator that had been removed from a nuclear power plant. The steam generator was removed before 1990 and the facility is no longer used.

- 377-SGEF. This stack exhausts filtered building entilation air. Particulate alpha and beta radioactivity sampling was conducted through March 8, 1990, and then discontinued.

1.2.4.19 3708 Radiation Measurements Laboratory. The building contains facilities for measuring small amounts of radionuclides, performing calibration studies, and leach studies with vitrified radioactive waste.

- EP-3708-01-S. This stack exhausts filtered building ventilation air. The exhaust is sampled for particulate alpha and beta radioactivity.

1.2.4.20 3720 Chemical and Metal Sciences Laboratory. The building contains low-level radiochemistry laboratories and a counting room.

- EP-3720-01-S. This stack exhausts filtered building air from the radiochemistry laboratories. The exhaust is sampled for particulate alpha and beta radioactivity.

- EP-3720-02-S. This stack exhausts filtered building air from the north-annex low-level radiochemistry laboratories. The exhaust is sampled for particulate alpha and beta radioactivity.

- EP-3720-03-S. This stack exhausts filtered building air from the south-end laboratories. This region of the building contains a counting room and lowlevel radiochemistry laboratories. The exhaust is sampled for particulate alpha and beta radioactivity.

1.2.4.21 3730 Gamma Irradiation Facility. The building contains a hot-cell for metallurgical studies of specimens of irradiated metals.

- EP-3730-01-S. This stack exhausts filtered building ventilation air. The exhaust is sampled for particulate alpha and beta radioactivity.

1.2.4.22 3745 Radiological Calibrations and Standards Facility. The building contains a counting room for measuring facility ventilation and exhaust samples.

- EP-3745-01-S. This stack exhausts filtered building ventilation air. The exhaust is sampled for particulate alpha and beta radioactivity. 
1.2.4.23 3746-A Radiological Physics Laboratory. The building contains facilities for low-level radiological physics studies.

- EP-3746A-01-V. This vent exhausts filtered building ventilation air. The exhaust is sampled for particulate alpha and beta radioactivity.

\subsubsection{Area Facilities}

The 400 Area consists of the FFTF, the Maintenance and Storage Facility (MASF), and the Fuels Materials Examination Facility. Emission points in the 400 Area are shown in Figure 1-7.

1.2.5.1 Fast Flux Test Facility. FFTF, located in the 400 Area, is a 400 -megawatt thermal, sodium-cooled, low-pressure, high-temperature reactor plant. It was used for irradiation testing of breeder reactor fuels and materials.

- $\quad$ FFTF-RE-SB. Lower Reactor Service Building (RSB). This exhaust discharges unfiltered air from the lower level of the RSB. Emission monitoring consists of a record sampler, a beta CAM, and a noble gas monitor.

- FFTF-CB-EX. Combined exhaust. This stack exhausts normally unfiltered air from the reactor containment and gases from the argon processing system. Standby particulate filters are automatically dampered into the system if airborne radioactive particulate levels rise above certain concentrations. Emission monitoring consists of a record sampler, a tritium sampler, a beta CAM, and a noble gas monitor.

- FFTF-HT-TR. Heat Transport System South. This exhaust discharges normally unfiltered air from portions of FFTF that are exterior to the containment. Standby particulate filters are automatically dampered into the system if airborne radioactive particulate levels rise above certain concentrations. Emission monitoring consists of a record sampler, a beta CAM, and a noble gas monitor.

1.2.5.2 437 MASF. The MASF is a multipurpose service center supporting the specialized maintenance and storage requirements of FFTF. The MASF provides the capability for sodium film removal, decontamination, repair, and storage of nonfueled components and hardware for FFTF.

- 437-MN\&ST. This exhaust discharges filtered air from the MASF. Emission monitoring consists of a record sampler and a beta CAM. 
Figure 1-7. 400 Area Emission Points.

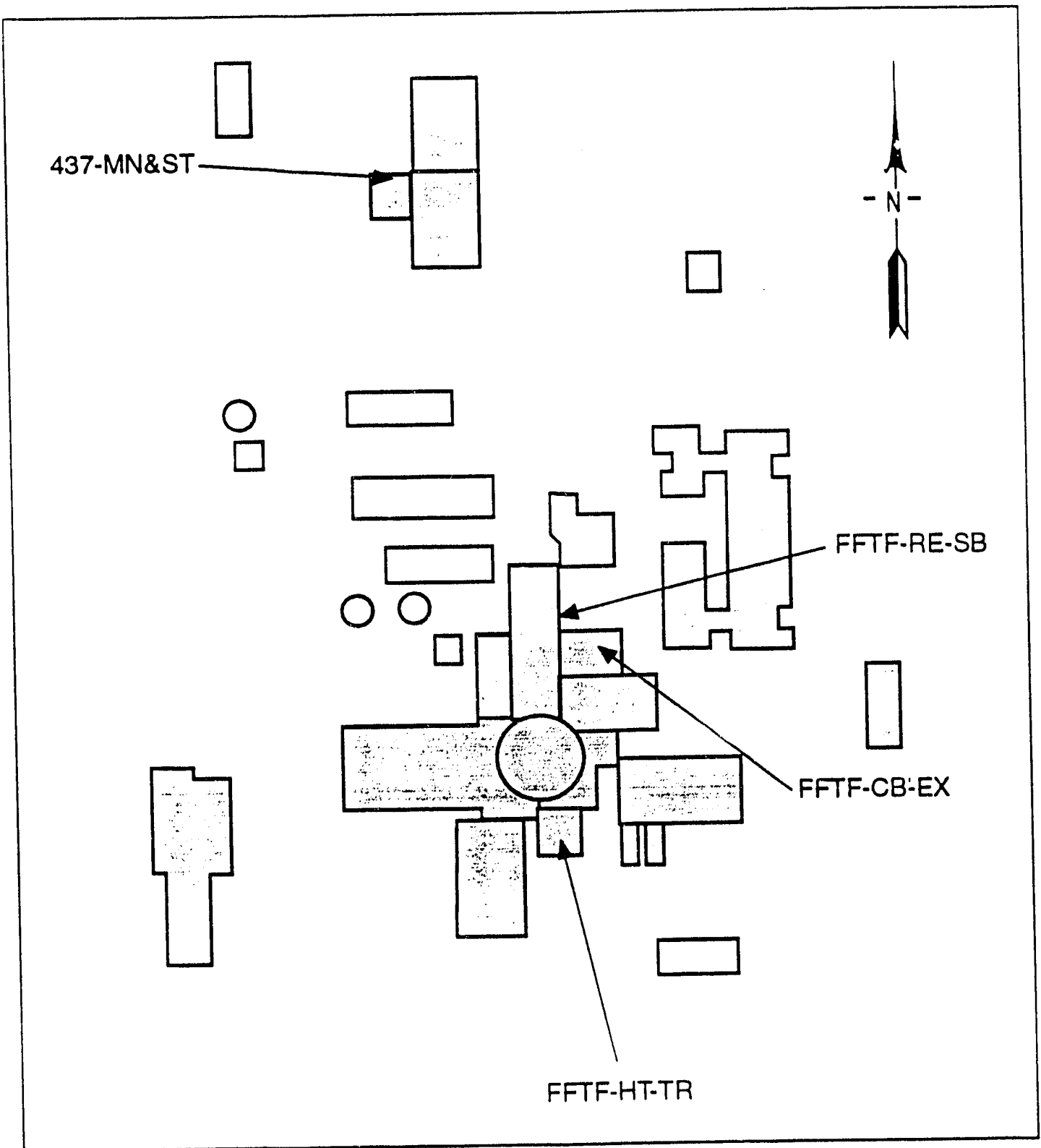




\subsubsection{Area Facilities}

Emission points in the 600 Area are shown in Figure 1-8.

1.2.6.1 6652-H Ecology Laboratory. This laboratory is located on the Arid Land Ecology Reserve. The building contains facilities for ecology studies.

- EP-6652H-01-V. This vent exhausts filtered building ventilation air. The exhaust has been sampled at two separate points (referred to as $6652 \mathrm{H}-01-\mathrm{V}$ and $6652 \mathrm{H}-02-\mathrm{V}$ ) for particulate alpha and beta radioactivity. Now only $6652 \mathrm{H}-01-\mathrm{V}$ is used. Sampling point $6652 \mathrm{H}-02-\mathrm{V}$ is registered unnecessarily with WDOH, who in 1993 will be requested to deregister it. Proper registration of the vent will be requested, too. WDOH has already been provided a full explanation on this vent and the two associated sampling points. 
Figure 1-8. 600 Area Emission Points.

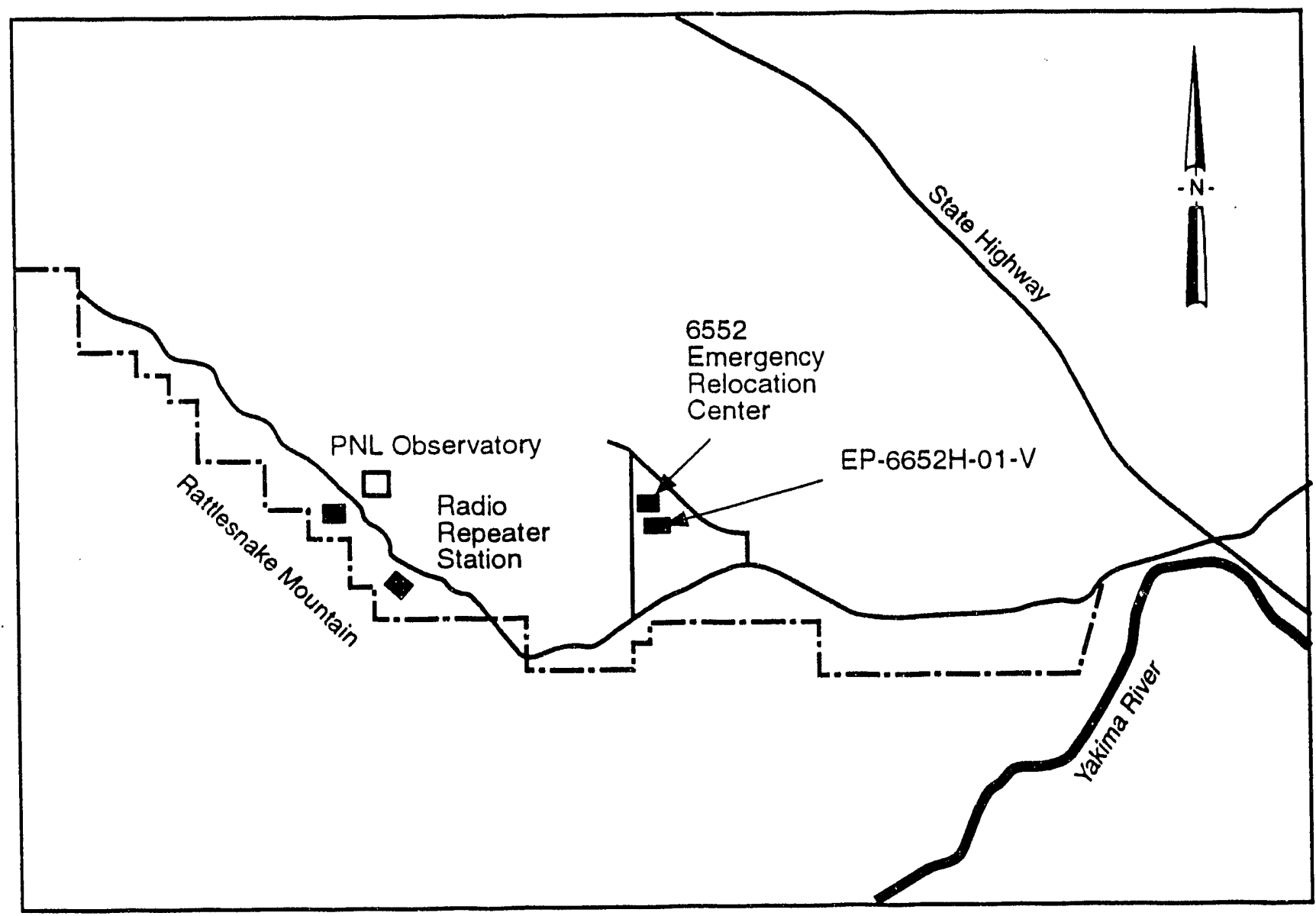

59305030.8 
DOE/RL-93-36

This page intentionally left blanl. 


\subsection{RADIONUCLIDE AIR EMISSIONS DATA ON POINT SOURCES}

Data on radionuclide emissions released during 1992 from the point sources (i.e., stacks) described in Section 1.2 are shown in Table 2-1. The table first displays source data on the 12 major Hanford Site stacks. A stack is classified as major if its emissions, with all treatment controls removed, have the potential to exceed $0.1 \mathrm{mrem} / \mathrm{yr}$ effective dose equivalent (EDE) at the nearest residence. A nearest residence is a known habitable location not on the Hanford Site that is nearest to an emission point representative of a collective operating area such as the 100 Areas. The maximally exposed individual (MEI) differs from a person at a nearest residence in that the MEI is a hypothetical member of the public residing near the Hanford Site who, by virtue of location (Ringold, Washington) and living habits, could receive the highest possible radiation dose from all radionuclide emissions from the Hanford Site.

The remainder of the table, beginning on page 2-4 and grouped according to the stack operating areas, gives data on an additional 120 minor stacks. A stack is considered minor when its potential m'ximum emissions with all treatment controls hypothetically removed cannot cause a dose at the nearest residence to exceed $0.1 \mathrm{mrem} / \mathrm{yr}$ EDE. All major and minor stack emissions in 1992 combined resulted in doses well below $0.1 \mathrm{mrem} / \mathrm{yr} \mathrm{EDE}$.

Each emission point listed has information on discharge height, distance to the nearest offsite residence, type and efficiency of any emission control, and the 1992 releases, in curies, by radionuclide or type of radioactivity.

Distances to the nearest offsite residence from reference points for stacks on the Hanford Site are listed in Table 2-2. A nearest residence is a dwelling, business, school, or office closest to a specific stack. The stacks are grouped by operating area, resulting in a single distance applicable to all the stacks in that area. Reporting these distances is a requirement of 40 CFR 61 (EPA 1992).

The distances to the respective nearest residences, and not 'he MEI, were used in determining whether a stack was major or minor (WHC 1991a). Thise distances, however, were not used in calculating the EDEs reported in this document. Instead, the distances from each operating area to the MEI location at the farm in Ringold, Washington, were used in the EDE calculations. The MEI location represents the receptor point for maximizing the dose from the total combined emissions of all Hanford Site stacks, not just those in individual operating areas.

An example of the difference between the two sets of distances is for the 200 West Area. The distance from 200 West Area stacks to the nearest residence is $15 \mathrm{mi}(24 \mathrm{~km})$ east. But the distance from 200 West Area stacks to the Hanford Site MEI location at the Ringold farm is $16 \mathrm{mi}(26 \mathrm{~km})$ east-southeast. 
Table 2-1. Hanford Site Radionuclide Air Emissions Data for 1992. (9 sheets)

\begin{tabular}{|c|c|c|c|c|c|c|c|}
\hline \multicolumn{8}{|c|}{ Major Stack (has potential of $>0.1 \mathrm{mrem} / \mathrm{yr}$ EDE to nearest offsite residence ${ }^{\mathrm{a}}$ ) } \\
\hline $\begin{array}{c}\text { ID }^{b} \\
\text { (location) }\end{array}$ & $\begin{array}{l}\text { Discharge } \\
\text { Height } \\
\text { (m) }\end{array}$ & $\begin{array}{c}\text { Nearest } \\
\text { Residence }^{c}\end{array}$ & $\begin{array}{l}\text { Emission } \\
\text { Control }^{d}\end{array}$ & $\begin{array}{c}\text { Total Flow } \\
\left(\mathrm{m}^{3}\right)\end{array}$ & Radionuclide & Curies & $\begin{array}{c}\mathrm{EDE}^{\mathrm{e}} \\
(\mathrm{mrem} / \mathrm{yr})\end{array}$ \\
\hline $\begin{array}{l}\text { 291-A-1 } \\
\text { (PUREX Plant, } \\
200 \text { East Area) }\end{array}$ & 61.0 & $16 \mathrm{~km}$ east & $\begin{array}{l}\text { HEPA, } \\
\text { charcoal }\end{array}$ & $1.6 \mathrm{E}+09$ & $\begin{array}{l}{ }^{90} \mathrm{Sr} \\
{ }^{129} \mathrm{I} \\
{ }^{137} \mathrm{Cs} \\
{ }^{147} \mathrm{Pm} \\
{ }^{212} \mathrm{~Pb} \\
{ }^{200} \mathrm{Rn} \\
{ }^{250} \mathrm{Pu} \\
{ }^{239.210} \mathrm{Pu} \\
{ }^{241} \mathrm{Pu} \\
{ }^{211} \mathrm{Am}\end{array}$ & $\begin{array}{l}3.3 \mathrm{E}-04 \\
2.7 \mathrm{E}-02 \\
1.6 \mathrm{E}-03 \\
1.7 \mathrm{E}-04 \\
2.7 \mathrm{E}-03 \\
3.4 \mathrm{E}+01 \\
1.3 \mathrm{E}-05 \\
2.2 \mathrm{E}-05 \\
4.7 \mathrm{E}-04 \\
1.3 \mathrm{E}-04\end{array}$ & $\begin{array}{c}1.5 \mathrm{E}-06 \\
1.8 \mathrm{E}-03 \\
2.4 \mathrm{E}-06 \\
2.0 \mathrm{E}-08 \\
9.8 \mathrm{E}-07 \\
- \\
1.1 \mathrm{E}-05 \\
2.0 \mathrm{E}-05 \\
6.8 \mathrm{E}-06 \\
1.8 \mathrm{E}-04\end{array}$ \\
\hline $\begin{array}{l}291-Z-1 \\
\text { (PFP, } \\
200 \text { West Area) }\end{array}$ & 61.0 & $24 \mathrm{~km}$ east & HEPA & $3.7 \mathrm{E}+09$ & $\begin{array}{l}{ }^{239,240} \mathrm{Pu} \\
{ }^{241} \mathrm{Pu} \\
{ }^{241} \mathrm{Am} \\
\text { total beta }\end{array}$ & $\begin{array}{l}4.2 \mathrm{E}-04 \\
2.9 \mathrm{E}-03 \\
7.2 \mathrm{E}-05 \\
3.4 \mathrm{E}-05\end{array}$ & $\begin{array}{l}3.8 \mathrm{E}-04 \\
4.2 \mathrm{E}-05 \\
9.9 \mathrm{E}-05 \\
1.6 \mathrm{E}-07\end{array}$ \\
\hline $\begin{array}{l}\text { 291-B-1 } \\
\text { (B Plant, } \\
200 \text { East Area) }\end{array}$ & 61.0 & $16 \mathrm{~km}$ east & HEPA & $7.2 E+08$ & $\begin{array}{l}{ }^{90} \mathrm{Sr} \\
{ }^{137} \mathrm{Cs} \\
{ }^{239.240} \mathrm{Pu}\end{array}$ & $\begin{array}{l}2.2 \mathrm{E}-05 \\
5.3 \mathrm{E}-04 \\
3.1 \mathrm{E}-06\end{array}$ & $\begin{array}{l}1.0 \mathrm{E}-07 \\
8.0 \mathrm{E}-07 \\
2.8 \mathrm{E}-06\end{array}$ \\
\hline $\begin{array}{l}291-\mathrm{T}-1 \\
\text { (T Plant, } \\
200 \text { West Area) }\end{array}$ & 61.0 & $24 \mathrm{~km}$ east & HEPA & $4.6 \mathrm{E}+08$ & $\begin{array}{l}{ }^{90} \mathrm{Sr} \\
{ }_{137} \mathrm{Cs} \\
{ }^{239,240} \mathrm{Pu} \\
{ }^{241} \mathrm{Am}\end{array}$ & $\begin{array}{l}1.2 \mathrm{E}-05 \\
1.3 \mathrm{E}-05 \\
2.2 \mathrm{E}-05 \\
2.0 \mathrm{E}-06\end{array}$ & $\begin{array}{l}5.5 \mathrm{E}-08 \\
2.0 \mathrm{E}-08 \\
2.0 \mathrm{E}-05 \\
2.8 \mathrm{E}-06\end{array}$ \\
\hline $\begin{array}{l}\text { 296-A-22 } \\
\text { (200-E Evaporator, } \\
200 \text { East Area) }\end{array}$ & 18.6 & $16 \mathrm{~km}$ east & HEPA & $1.1 E+07$ & $\begin{array}{l}\text { total alpha } \\
\text { total beta }\end{array}$ & $\begin{array}{l}5.6 \mathrm{E}-09 \\
9.1 \mathrm{E}-07\end{array}$ & $\begin{array}{ll}5.1 & E-09 \\
4.2 & E-09\end{array}$ \\
\hline $\begin{array}{l}296-A-40 \\
\text { (241-AP Tank Farm, } \\
200 \text { East Area) }\end{array}$ & 4.1 & $16 \mathrm{~km}$ east & HEPA & $1.8 \mathrm{E}+07$ & $\begin{array}{l}{ }^{90} \mathrm{Sr} \\
\text { total alpha }\end{array}$ & $\begin{array}{c}3.2 E-08 \\
N D^{f}\end{array}$ & $\begin{array}{c}1.5 \mathrm{E}-10 \\
-\end{array}$ \\
\hline $\begin{array}{l}\text { 340-NT-EX } \\
\text { (340 Waste Handling, } \\
300 \text { Area) }\end{array}$ & 5.5 & $\begin{array}{c}1.4 \mathrm{~km} \\
\text { northeast }\end{array}$ & $\begin{array}{c}\text { HEPA, } \\
\text { charcoal }\end{array}$ & $2.5 \mathrm{E}+07$ & $\begin{array}{l}\text { totill alpha } \\
\text { tolal beta }\end{array}$ & $\begin{array}{l}2.1 E-08 \\
8.9 E-08\end{array}$ & $\begin{array}{ll}2.1 & E-07 \\
4.5 & E-09\end{array}$ \\
\hline $\begin{array}{l}\text { EP-324-01-S } \\
\text { (324 Bldg., } \\
300 \text { Area) }\end{array}$ & 48.4 & $\begin{array}{l}1.9 \mathrm{~km} \\
\text { south }\end{array}$ & HEPA & $1.4 E+09$ & $\begin{array}{l}{ }^{3} \mathrm{H} \text { (as HTO) } \\
{ }^{3} \mathrm{H} \text { (as HT) } \\
\text { total alpha } \\
\text { total beta }\end{array}$ & $\begin{array}{l}9.6 E+00 \\
2.2 E+00 \\
3.9 E-07 \\
6.4 E-07\end{array}$ & $\begin{array}{l}8.2 \mathrm{E}-05 \\
1.9 \mathrm{E}-07 \\
1.4 \mathrm{E}-06 \\
1.2 \mathrm{E}-08\end{array}$ \\
\hline $\begin{array}{l}\text { EP-325-01-S } \\
\text { (325 Bldg., } \\
300 \text { Area) }\end{array}$ & 17.9 & $\begin{array}{l}1.9 \mathrm{~km} \\
\text { south }\end{array}$ & HEPA & $1.8 E+09$ & $\begin{array}{l}{ }^{3} \mathrm{H} \text { (as } \mathrm{HTO}^{8} \\
{ }^{3} \mathrm{H} \text { (as HT) } \\
\text { total alpha } \\
\text { total beta }\end{array}$ & $\begin{array}{l}2.5 \mathrm{E}+01 \\
7.0 \mathrm{E}+00 \\
8.5 \mathrm{E}-07 \\
2.4 \mathrm{E}-06\end{array}$ & $\begin{array}{l}6.7 \mathrm{E}-04 \\
1.9 \mathrm{E}-06 \\
8.4 \mathrm{E}-06 \\
1.2 \mathrm{E}-07\end{array}$ \\
\hline $\begin{array}{l}\text { EP-327-01-S } \\
\text { (327 Bldg., } \\
300 \text { Area) }\end{array}$ & 13.7 & $\begin{array}{l}1.9 \mathrm{~km} \\
\text { south }\end{array}$ & HEPA & $7.7 E+08$ & $\begin{array}{l}\text { total alpha } \\
\text { total beta }\end{array}$ & $\begin{array}{l}1.1 E-07 \\
9.3 E-07\end{array}$ & $\begin{array}{l}1.1 E-06 \\
4.7 E-08\end{array}$ \\
\hline $\begin{array}{l}\text { EP-327-02-V } \\
\text { (327 Decon. Cell, } \\
300 \text { Area) }\end{array}$ & 9.1 & $\begin{array}{l}1.9 \mathrm{~km} \\
\text { south }\end{array}$ & HEPA & $7.9 E+06$ & $\begin{array}{l}\text { total alpha } \\
\text { total beta }\end{array}$ & $\begin{array}{l}1.8 \mathrm{E}-09 \\
1.1 \mathrm{E}-08\end{array}$ & $\begin{array}{l}1.8 \mathrm{E}-08 \\
5.5 \mathrm{E}-10\end{array}$ \\
\hline
\end{tabular}


Table 2-1. Hanford Site Radionuclide Air Emissions Data for 1992. (9 sheets)

\begin{tabular}{|l|c|c|c|c|c|c|c|}
\hline \multicolumn{7}{|c|}{ Major Stack (has potential of $>0.1 \mathrm{mrem} / \mathrm{yr}$ EDE to nearest offsite residence) } \\
\hline $\begin{array}{c}\text { ID } \\
\text { (location) }\end{array}$ & $\begin{array}{c}\text { Discharge } \\
\text { Ileight } \\
(\mathrm{m})\end{array}$ & $\begin{array}{c}\text { Nearest } \\
\text { Residence }\end{array}$ & $\begin{array}{c}\text { Emission } \\
\text { Control }\end{array}$ & $\begin{array}{c}\text { Total Flow } \\
\left(\mathrm{m}^{3}\right)\end{array}$ & Radionuclide & Curies & $\begin{array}{c}\text { EDE } \\
(\mathrm{mrem} / \mathrm{yr})\end{array}$ \\
\hline $\begin{array}{l}\text { EP-3720-01-S } \\
(3720 \text { Bldg., } \\
300 \text { Area) }\end{array}$ & 9.1 & $\begin{array}{c}1.9 \mathrm{~km} \\
\text { south }\end{array}$ & HEPA & $4.3 \mathrm{E}+08$ & $\begin{array}{l}\text { total alpha } \\
\text { total beta }\end{array}$ & $\begin{array}{l}5.0 \mathrm{E}-08 \\
3.8 \mathrm{E}-08\end{array}$ & $\begin{array}{c}5.0 \mathrm{E}-07 \\
1.9 \mathrm{E}-09\end{array}$ \\
\hline
\end{tabular}


Table 2-1. Hanford Site Radionuclide Air Emissions Data for 1992. (9 sheets)

\begin{tabular}{|c|c|c|c|c|c|}
\hline \multicolumn{6}{|c|}{ Minor Stack (has maximum dose potential of $<0.1 \mathrm{mrem} / \mathrm{yr}$ EDE to nearest offsite residence ${ }^{\mathrm{a}}$ ) } \\
\hline $\begin{array}{c}\mathrm{ID}^{\mathrm{b}} \\
\text { (location) }\end{array}$ & $\begin{array}{l}\text { Discharge } \\
\text { Height (m) }\end{array}$ & $\begin{array}{l}\text { Emission } \\
\text { Control }^{d}\end{array}$ & $\begin{array}{c}\text { Total Flow } \\
\left(\mathrm{m}^{3}\right)\end{array}$ & Radionuclide & Curies \\
\hline \multicolumn{6}{|c|}{100 Areas Stacks } \\
\hline $\begin{array}{l}116-N \\
(100-N \text { Area })\end{array}$ & 61.3 & $\begin{array}{l}\text { HEPA, } \\
\text { charcoal }\end{array}$ & $1.0 \mathrm{E}+09$ & $\begin{array}{l}{ }^{60} \mathrm{Co} \\
{ }^{90} \mathrm{Sr} \\
{ }^{106} \mathrm{Ru} \\
{ }^{137} \mathrm{Cs} \\
{ }^{239,210} \mathrm{Pu}\end{array}$ & $\begin{array}{l}4.7 \mathrm{E}-07 \\
2.7 \mathrm{E}-06 \\
1.7 \mathrm{E}-05 \\
1.9 \mathrm{E}-06 \\
1.0 \mathrm{E}-08\end{array}$ \\
\hline $\begin{array}{l}\text { 109-N-Z-I } \\
\text { (100-N Area) }\end{array}$ & 13.7 & none & \multicolumn{3}{|c|}{ (did not operate) } \\
\hline $\begin{array}{l}109-N-C-6 \\
(100-N \text { Area) }\end{array}$ & 13.7 & none & \multicolumn{3}{|c|}{ (did not operate) } \\
\hline $\begin{array}{l}\text { 105-N 14-ft } \\
\text { Decontamination Room } \\
\text { (100-N Area); not registered } \\
\text { with WDOH }\end{array}$ & 8.5 & HEPA & $9.6 \mathrm{E}+07$ & $\begin{array}{l}{ }^{60} \mathrm{Co} \\
{ }^{90} \mathrm{Sr} \\
{ }^{125} \mathrm{Sb} \\
{ }^{137} \mathrm{Cs} \\
{ }^{154} \mathrm{Eu} \\
{ }^{288} \mathrm{Pu} \\
{ }^{239,240} \mathrm{Pu}\end{array}$ & $\begin{array}{l}5.5 \mathrm{E}-07 \\
7.0 \mathrm{E}-08 \\
2.6 \mathrm{E}-07 \\
1.6 \mathrm{E}-07 \\
9.7 \mathrm{E}-08 \\
2.4 \mathrm{E}-09 \\
1.4 \mathrm{E}-08\end{array}$ \\
\hline $\begin{array}{l}\text { 105-N Transfer Area } \\
\text { (100-N Area); not registered } \\
\text { with WDOH }\end{array}$ & 18 & none & $4.1 \mathrm{E}+08$ & $\begin{array}{l}{ }^{137} \mathrm{Cs} \\
{ }^{239,210} \mathrm{Pu} \\
\text { total beta }\end{array}$ & $\begin{array}{l}8.0 \mathrm{E}-07 \\
2.3 \mathrm{E}-09 \\
\quad \mathrm{ND}\end{array}$ \\
\hline $\begin{array}{l}\text { 107-N Basin Recirculation } \\
\text { Facility } \\
\text { (100-N Area); not registered } \\
\text { with WDOH }\end{array}$ & 12 & HEPA & $1.1 \mathrm{E}+08$ & $\begin{array}{l}{ }^{60} \mathrm{Co} \\
{ }^{90} \mathrm{Sr} \\
{ }^{106} \mathrm{Ru} \\
{ }^{125} \mathrm{Ru} \\
{ }^{154} \mathrm{Eu} \\
{ }^{238} \mathrm{Pu} \\
{ }^{239.240} \mathrm{Pu}\end{array}$ & $\begin{array}{l}4.6 \mathrm{E}-08 \\
2.7 \mathrm{E}-08 \\
8.6 \mathrm{E}-07 \\
7.7 \mathrm{E}-08 \\
4.0 \mathrm{E}-07 \\
6.8 \mathrm{E}-10 \\
1.7 \mathrm{E}-09\end{array}$ \\
\hline $\begin{array}{l}\text { 105-KE } \\
\text { (KE Basin, } \\
\text { 100-KE Area) }\end{array}$ & 12.8 & none & $7.5 \mathrm{E}+08$ & $\begin{array}{l}{ }^{60} \mathrm{Co} \\
{ }^{90} \mathrm{Sr} \\
{ }^{106} \mathrm{Ru} \\
{ }^{125} \mathrm{Sb} \\
{ }^{137} \mathrm{Cs} \\
{ }^{238} \mathrm{Pu} \\
{ }^{239,240} \mathrm{Pu} \\
{ }^{201} \mathrm{Pu} \\
{ }^{241} \mathrm{Am}\end{array}$ & $\begin{array}{ll}1.3 & E-06 \\
1.6 & E-04 \\
1.3 & E-05 \\
1.1 & E-05 \\
2.3 & E-04 \\
1.3 & E-06 \\
8.5 & E-06 \\
3.9 & E-05 \\
5.1 & E-06\end{array}$ \\
\hline $\begin{array}{l}\text { 105-KW } \\
\text { (KW Basin, } \\
\text { 100-KW Area) }\end{array}$ & 12.8 & none & $7.5 \mathrm{E}+08$ & $\begin{array}{l}{ }^{60} \mathrm{Co} \\
{ }^{90} \mathrm{Sr} \\
{ }^{106} \mathrm{Ru} \\
{ }^{137} \mathrm{Cs} \\
{ }^{154} \mathrm{Eu} \\
{ }^{238} \mathrm{Pu} \\
{ }^{239.240} \mathrm{Pu}\end{array}$ & $\begin{array}{l}1.4 \mathrm{E}-06 \\
9.9 \mathrm{E}-07 \\
6.2 \mathrm{E}-06 \\
2.7 \mathrm{E}-05 \\
4.9 \mathrm{E}-06 \\
3.0 \mathrm{E}-08 \\
1.8 \mathrm{E}-07\end{array}$ \\
\hline
\end{tabular}


Table 2-1. Hanford Site Radionuclide Air Emissions Data for 1992. (9 sheets)

\begin{tabular}{|c|c|c|c|c|c|}
\hline \multicolumn{6}{|c|}{ Minor Stack (has maximum dose potential of $<0.1 \mathrm{mrem} / \mathrm{yr}$ EDE to nearest offsite residence ${ }^{\mathrm{a}}$ ) } \\
\hline $\begin{array}{c}\mathrm{ID}^{\mathrm{b}} \\
\text { (location) }\end{array}$ & $\begin{array}{l}\text { Discharge } \\
\text { lleight (m) }\end{array}$ & $\begin{array}{l}\text { Emission } \\
\text { Control }^{d}\end{array}$ & $\begin{array}{l}\text { Total Flow } \\
\left(\mathrm{mn}^{3}\right)\end{array}$ & Radionuclide & Curies \\
\hline \multicolumn{6}{|c|}{100 Areas Stacks (continued) } \\
\hline $\begin{array}{l}\text { 1706-KER } \\
\text { (-27-ft Laboratory } \\
\text { Basement, 100-KE Area); } \\
\text { not registered with WDOH }\end{array}$ & 0.9 & HEPA & \multicolumn{3}{|c|}{ (did not operate) } \\
\hline $\begin{array}{l}\text { 1706-KE } \\
\text { (Laboratory, } \\
\text { 100-KE Area) }\end{array}$ & 7.6 & HEPA & $1.8 \mathrm{E}+08$ & $\begin{array}{l}{ }^{20} \mathrm{Sr} \\
{ }^{106} \mathrm{Ru} \\
{ }^{208} \mathrm{Pu} \\
{ }^{209.210} \mathrm{Pu}\end{array}$ & $\begin{array}{l}1.0 \mathrm{E}-07 \\
6.2 \mathrm{E}-08 \\
4.5 \mathrm{E}-10 \\
2.5 \mathrm{E}-09\end{array}$ \\
\hline \multicolumn{6}{|c|}{200 East Area Stacks } \\
\hline $\begin{array}{l}\text { 296-A-1 } \\
\text { (PUREX Plant) }\end{array}$ & 20.7 & HEPA & $6.1 \mathrm{E}+07$ & $\begin{array}{l}{ }^{239.240} \mathrm{Pu} \\
{ }^{241} \mathrm{Pu} \\
{ }^{241} \mathrm{Am} \\
\text { total beta }\end{array}$ & $\begin{array}{c}1.0 \mathrm{E}-06 \\
7.1 \mathrm{E}-06 \\
3.5 \mathrm{E}-07 \\
\mathrm{ND}\end{array}$ \\
\hline 296-A-2 (PUREX Plant) & 20.7 & HEPA & $6.1 \mathrm{E}+07$ & \multicolumn{2}{|c|}{ ND } \\
\hline 296-A-3 (PUREX Plant) & 24.1 & HEPA & $3.6 \mathrm{E}+07$ & \multicolumn{2}{|c|}{ ND } \\
\hline 296-A-5A (PUREX Plant) & 17.1 & HEPA & $3.4 \mathrm{E}+08$ & $\begin{array}{l}239,240 \mathrm{Pu} \\
\text { total beta }\end{array}$ & $\begin{array}{c}6.4 \mathrm{E}-07 \\
\mathrm{ND}\end{array}$ \\
\hline 296-A-5B (PUREX Plant) & 17.1 & HEPA & $3.4 \mathrm{E}+08$ & $\begin{array}{l}{ }^{239,240} \mathrm{Pu} \\
\text { total beta }\end{array}$ & $\begin{array}{c}4.1 \mathrm{E}-07 \\
\mathrm{ND}\end{array}$ \\
\hline 296-A-6 (PỤREX Plant) & 20.7 & HEPA & $2.0 \mathrm{E}+08$ & \multicolumn{2}{|c|}{ ND } \\
\hline 296-A-7 (PUREX Plant) & 20.7 & HEPA & $2.7 \mathrm{E}+08$ & \multicolumn{2}{|c|}{ ND } \\
\hline 296-A-8 (PUREX Plant) & 20.7 & HEPA & $1.4 \mathrm{E}+08$ & \multicolumn{2}{|c|}{ ND } \\
\hline 296-A-10 (PUREX Plant) & 4.6 & HEPA & $8.3 E+07$ & \multicolumn{2}{|c|}{ ND } \\
\hline 296-A-14 (PUREX Plant) & 9.1 & HEPA & $7.0 \mathrm{E}+07$ & \multicolumn{2}{|c|}{ ND } \\
\hline 296-A-24 (PUREX Plant) & 20.7 & HEPA & \multicolumn{3}{|c|}{ (did not operate) } \\
\hline 296-A-33 (PUREX Plant) & 6.1 & none & \multicolumn{3}{|c|}{ (did not operate) } \\
\hline 296-A-34 (PUREX Plant) & 6.1 & none & \multicolumn{3}{|c|}{ (did not operate) } \\
\hline 296-A-35 (PUREX Plant) & 6.1 & none & \multicolumn{3}{|c|}{ (did not operate) } \\
\hline 296-A-36 (PUREX Plant) & 6.1 & none & \multicolumn{3}{|c|}{ (did not operate) } \\
\hline 296-A-37 (PUREX Plant) & 6.1 & none & \multicolumn{3}{|c|}{ (did not operate) } \\
\hline 296-A-38 (PUREX Plant) & 6.1 & none & $1.5 \mathrm{E}+06$ & $\begin{array}{l}\text { total alpha } \\
\text { total beta }\end{array}$ & $\begin{array}{c}\mathrm{ND} \\
3.1 \mathrm{E}-08\end{array}$ \\
\hline 296-B-5 (B Plant) & 3.7 & HEPA & $1.8 \mathrm{E}+07$ & \multicolumn{2}{|c|}{ ND } \\
\hline
\end{tabular}


Table 2-1. Hanford Site Radionuclide Air Emissions Data for 1992. (9 sheets)

\begin{tabular}{|c|c|c|c|c|c|}
\hline \multicolumn{6}{|c|}{ Minor Stack (has maximum dose potential of $<0.1 \mathrm{mrem} / \mathrm{yr}$ EDE to nearest offsite residence ${ }^{\mathrm{a}}$ ) } \\
\hline $\begin{array}{c}\mathrm{ID}^{\mathrm{b}} \\
\text { (location) }\end{array}$ & $\begin{array}{l}\text { Discharge } \\
\text { Height (m) }\end{array}$ & $\begin{array}{l}\text { Einission } \\
\text { Control }^{d}\end{array}$ & $\begin{array}{l}\text { Total Flow } \\
\left(\mathrm{wn}^{3}\right)\end{array}$ & Radionuclide & Curies \\
\hline \multicolumn{6}{|c|}{200 East Area Stacks (continued) } \\
\hline 296-B-10 (B Plant) & 22.9 & HEPA & $2.9 \mathrm{E}+08$ & $\begin{array}{l}90 \mathrm{Sr} \\
\text { total alpha }\end{array}$ & $\begin{array}{c}2.5 \mathrm{E}-06 \\
\mathrm{ND}\end{array}$ \\
\hline 296-B-13 (B Plant) & 3.5 & HEPA & $1.1 \mathrm{E}+07$ & \multicolumn{2}{|c|}{ ND } \\
\hline 296-B-14 (B Plant) & 0.0 & HEPA & \multicolumn{3}{|c|}{ (did not operate) } \\
\hline 296-A-12 (AR Vault) & 45.7 & HEPA & \multicolumn{3}{|c|}{$\begin{array}{l}\text { (operated }<48 \mathrm{hr} \text { for year; } \\
\text { valid data not obtainable) }\end{array}$} \\
\hline 296-A-13 (AR Vault) & 38.1 & HEPA & $7.2 \mathrm{E}+07$ & \multicolumn{2}{|c|}{ ND } \\
\hline 296-C-5 (CR Vault) & 14.6 & HEPA & $2.4 E+07$ & $\begin{array}{l}\text { total alpha } \\
\text { total beta }\end{array}$ & $\begin{array}{l}2.9 \mathrm{E}-09 \\
2.7 \mathrm{E}-07\end{array}$ \\
\hline 296-A-17 (Tank Farms) & 15.2 & HEPA & $4.9 E+07$ & $\begin{array}{l}{ }^{90} \mathrm{Sr} \\
{ }^{129} \mathrm{I} \\
{ }^{137} \mathrm{Cs} \\
\text { total alpha }\end{array}$ & $\begin{array}{c}4.6 E-07 \\
1.5 E-04 \\
4.0 E-06 \\
N D\end{array}$ \\
\hline 296-A-18 (Tank Farms) & 4.6 & HEPA & \multicolumn{3}{|c|}{ (did not operate) } \\
\hline 296-A-19 (Tank Farms) & 4.6 & HEPA & \multicolumn{3}{|c|}{ (did not operate) } \\
\hline 296-A-20 (Tank Farms) & 7.3 & HEPA & $2.9 \mathrm{E}+06$ & \multicolumn{2}{|c|}{ ND } \\
\hline 296-A-21 (Evaporator) & 6.7 & HEPA & $2.9 \mathrm{E}+08$ & \multicolumn{2}{|c|}{ ND } \\
\hline 296-A-25 (Tank Farms) & 3.0 & HEPA & $3.6 \mathrm{E}+05$ & \multicolumn{2}{|c|}{ ND } \\
\hline 296-A-26 (T ank Farms) & 9.4 & HEPA & $2.6 \mathrm{E}+07$ & \multicolumn{2}{|c|}{ ND } \\
\hline 296-A-27 (Tank Farms) & 3.7 & H 'A & $2.1 \mathrm{E}+07$ & $\begin{array}{l}{ }^{90} \mathrm{Sr} \\
\text { total alpha }\end{array}$ & $\begin{array}{l}4.2 \mathrm{E}-08 \\
\mathrm{ND}\end{array}$ \\
\hline 296-A-28 (Tank Farms) & 3.7 & HEPA & $6.6 E+07$ & \multicolumn{2}{|c|}{ ND } \\
\hline 296-A-29 (Tank Farms) & 3.7 & HEPA & $1.0 \mathrm{E}+07$ & $\begin{array}{l}\text { total alpha } \\
\text { total beta }\end{array}$ & $\begin{array}{c}N D \\
4.1 \mathrm{E}-06\end{array}$ \\
\hline 296-A-30 (Tank Farms) & 3.7 & HEPA & $7.2 \mathrm{E}+07$ & \multicolumn{2}{|c|}{ ND } \\
\hline 296-A-41 (Tank Farms) & 8.9 & HEPA & $5.0 \mathrm{E}+07$ & \multicolumn{2}{|c|}{ ND } \\
\hline 296-B-28 (Tank Farms) & 3.4 & HEPA & $2.9 \mathrm{E}+05$ & $\begin{array}{l}\text { total alpha } \\
\text { total beta }\end{array}$ & $\begin{array}{l}6.0 \mathrm{E}-10 \\
2.4 \mathrm{E}-08\end{array}$ \\
\hline 296-P-16 (Tank Farms) & 4.6 & HEPA & $2.7 \mathrm{E}+07$ & $\begin{array}{l}\text { total alpha } \\
\text { total beta }\end{array}$ & $\begin{array}{l}4.1 \mathrm{E}-09 \\
1.3 \mathrm{E}-05\end{array}$ \\
\hline 296-P-17 (Tank Farms) & 4.6 & HEPA & $3.0 \mathrm{E}+05$ & $\begin{array}{l}\text { total alpha } \\
\text { total beta }\end{array}$ & $\begin{array}{c}\mathrm{ND} \\
2.6 \mathrm{E}-08\end{array}$ \\
\hline
\end{tabular}


Table 2-1. Hanford Site Radionuclide Air Emissions Data for 1992. (9 sheets)

\begin{tabular}{|c|c|c|c|c|c|}
\hline \multicolumn{6}{|c|}{ Minor Stack (has maximum dose potential of $<0.1 \mathrm{mrem} / \mathrm{yr}$ EDE to nearest offsite residence ${ }^{\mathrm{a}}$ ) } \\
\hline $\begin{array}{c}\mathrm{ID}^{\mathrm{b}} \\
\text { (location) }\end{array}$ & $\begin{array}{l}\text { Discharge } \\
\text { Ileight (m) }\end{array}$ & $\begin{array}{l}\text { Emission } \\
\text { Control }^{d}\end{array}$ & $\begin{array}{c}\text { Total Flow } \\
\left(\mathrm{m}^{3}\right)\end{array}$ & Radionuclide & Curies \\
\hline \multicolumn{6}{|c|}{200 East Area Stacks (continued) } \\
\hline 29.6-G-1 (Grout) & 7.6 & HEPA & $2.1 \mathrm{E}+06$ & \multicolumn{2}{|c|}{ ND } \\
\hline :96-P-31 (209-E Bldg.) & 10 & HEPA & \multicolumn{3}{|c|}{ (operate briefly; valid data not obtainable) } \\
\hline \multicolumn{6}{|c|}{200 West Area Stacks } \\
\hline 291-S-1 (S Plant) & 61.0 & sand filter & $2.7 \mathrm{E}+08$ & $\begin{array}{l}239.240 \mathrm{Pu} \\
\text { total beta }\end{array}$ & $\begin{array}{l}2.7 \mathrm{E}-07 \\
1.0 \mathrm{E}-08\end{array}$ \\
\hline 296-S-2 (S Plant) & 20.7 & HEPA & $8.8 \mathrm{E}+06$ & $\begin{array}{l}\text { total alpha } \\
\text { total beta }\end{array}$ & $\begin{array}{l}8.4 \mathrm{E}-09 \\
1.4 \mathrm{E}-08\end{array}$ \\
\hline 296-S-4 (S Plant) & 20.7 & HEPA & $4.9 E+07$ & $\begin{array}{l}\text { total alpha } \\
\text { total beta }\end{array}$ & $\begin{array}{l}7.5 E-10 \\
5.1 E-07\end{array}$ \\
\hline 296-S-6 (S Plant) & 20.7 & none & $1.4 \mathrm{E}+08$ & $\begin{array}{l}\text { total alpha } \\
\text { total beta }\end{array}$ & $\begin{array}{l}2.0 \mathrm{E}-07 \\
3.9 \mathrm{E}-06\end{array}$ \\
\hline 296-S-7W (S Plant) & 7.6 & HEPA & $1.3 \mathrm{E}+08$ & $\begin{array}{l}{ }^{239.240} \mathrm{Pu} \\
{ }^{241} \mathrm{Am} \\
\text { total beta }\end{array}$ & $\begin{array}{c}7.5 \mathrm{E}-07 \\
4.2 \mathrm{E}-06 \\
\mathrm{ND}\end{array}$ \\
\hline 296-T-11 (T Plant) & 7.6 & HEPA & $2.4 \mathrm{E}+08$ & $\begin{array}{l}\text { total alpha } \\
\text { total beta }\end{array}$ & $\begin{array}{c}\text { ND } \\
4.3 E-07\end{array}$ \\
\hline 296-T-12 (T Plant) & 7.6 & HEPA & $5.1 \mathrm{E}+07$ & \multicolumn{2}{|c|}{ ND } \\
\hline 296-T-13 (T Plant) & 20.7 & HEPA & $4.3 \mathrm{E}+08$ & $\begin{array}{l}239,240 \mathrm{Pu} \\
\text { total beta }\end{array}$ & $\begin{array}{l}2.0 \mathrm{E}-06 \\
5.0 \mathrm{E}-06\end{array}$ \\
\hline 291-U-1 (U Plant) & 61.0 & sand filter & $3.1 \mathrm{E}+08$ & $\begin{array}{l}{ }^{90} \mathrm{Sr} \\
{ }^{137} \mathrm{Cs} \\
{ }^{239,240} \mathrm{Pu}\end{array}$ & $\begin{array}{l}1.5 \mathrm{E}-05 \\
2.2 \mathrm{E}-04 \\
3.1 \mathrm{E}-07\end{array}$ \\
\hline 296-U-2 (U Plant) & 24.4 & HEPA & $6.5 \mathrm{E}+06$ & $\begin{array}{l}\text { total alpha } \\
\text { total beta }\end{array}$ & $\begin{array}{l}1.2 E-07 \\
1.3 E-07\end{array}$ \\
\hline $296-\mathrm{U}-4\left(\mathrm{UO}_{3}\right)$ & 42.7 & none & $3.2 \mathrm{E}+07$ & $\begin{array}{l}{ }^{90} \mathrm{Sr} \\
{ }^{137} \mathrm{Cs} \\
\mathrm{U}, \text { total } \\
{ }^{239.240} \mathrm{Pu}\end{array}$ & $\begin{array}{l}5.4 \mathrm{E}-08 \\
3.8 \mathrm{E}-07 \\
9.4 \mathrm{E}-07 \\
4.0 \mathrm{E}-08\end{array}$ \\
\hline 296-U-13 (U Plant) & 8.2 & HEPA & $4.2 \mathrm{E}+06$ & \multicolumn{2}{|c|}{ ND } \\
\hline 296-Z-3 (PFP) & 7.3 & HEPA & $3.6 \mathrm{E}+07$ & $\begin{array}{l}239,240 \mathrm{Pu} \\
{ }^{201} \mathrm{Am} \\
\text { total beta }\end{array}$ & $\begin{array}{l}1.0 \mathrm{E}-06 \\
8.8 \mathrm{E}-07 \\
2.8 \mathrm{E}-07\end{array}$ \\
\hline 296-Z-5 (PFP) & 4.6 & HEPA & $1.2 \mathrm{E}+08$ & \multicolumn{2}{|c|}{ ND } \\
\hline 296-Z-6 (PFP) & 0.9 & HEPA & $1.5 E+08$ & \multicolumn{2}{|c|}{ ND } \\
\hline 296-Z-10 (PFP) & 10 & HEPA & $3.7 \mathrm{E}+07$ & \multicolumn{2}{|c|}{ (not record sampled) } \\
\hline
\end{tabular}


Table 2-1. Hanford Site Radionuclide Air Emissions Data for 1992. (9 sheets)

\begin{tabular}{|c|c|c|c|c|c|}
\hline \multicolumn{6}{|c|}{ Minor Stack (has maximum dose potential of $<0.1 \mathrm{mrem} / \mathrm{yr}$ EDE to nearest offsite residence ${ }^{\mathrm{a}}$ ) } \\
\hline $\begin{array}{c}\mathrm{ID}^{\mathrm{b}} \\
\text { (location) }\end{array}$ & $\begin{array}{l}\text { Discharge } \\
\text { Height (m) }\end{array}$ & $\begin{array}{l}\text { Emission } \\
\text { Control }^{d}\end{array}$ & $\begin{array}{l}\text { Total Flow } \\
\quad\left(\mathrm{m}^{3}\right)\end{array}$ & Radionuclide & Curies \\
\hline \multicolumn{6}{|c|}{200 West Area Stacks (continued) } \\
\hline 296-Z-11 (PFP) & 4 & HEPA & $5.6 \mathrm{E}+07$ & (not reco & ampled) \\
\hline 296-Z-14 (PFP) & 6.1 & HEPA & $2.5 \mathrm{E}+07$ & $\begin{array}{l}\text { total alpha } \\
\text { total beta }\end{array}$ & $\begin{array}{c}3.8 \mathrm{E}-08 \\
\mathrm{ND}\end{array}$ \\
\hline 296-P-22 (Tank Farms) & 4.6 & HEPA & $1.4 \mathrm{E}+07$ & \multicolumn{2}{|c|}{ ND } \\
\hline 296-P-23 (Tank Farms) & 4.6 & HEPA & $1.3 \mathrm{E}+07$ & $\begin{array}{l}\text { total alpha } \\
\text { total beta }\end{array}$ & $\begin{array}{l}6.6 \mathrm{E}-10 \\
9.1 \mathrm{E}-07\end{array}$ \\
\hline 296-S-15 (Tank Farms) & 4.6 & HEPA & $3.8 \mathrm{E}+07$ & $\begin{array}{l}\text { total alpha } \\
\text { total beta }\end{array}$ & $\begin{array}{c}\text { ND } \\
1.1 \mathrm{E}-07\end{array}$ \\
\hline 296-S-18 (Evaporator) & 6.7 & HEPA & $3.0 \mathrm{E}+08$ & \multicolumn{2}{|c|}{ ND } \\
\hline 296-S-22 (Tank Farms) & 3.7 & HEPA & $4.4 E+06$ & \multicolumn{2}{|c|}{ ND } \\
\hline 296-T-17 (Evaporator) & 10.1 & HEPA & $2.4 \mathrm{E}+07$ & $\begin{array}{l}\text { total alpha } \\
\text { total beta }\end{array}$ & $\begin{array}{c}5.0 \mathrm{E}-10 \\
\mathrm{ND}\end{array}$ \\
\hline 296-T-18 (Tank Farms) & 3.7 & HEPA & $3.1 E+06$ & total beta & $3.0 \mathrm{E}-08$ \\
\hline 296-W-1 (Laundry) & 6.7 & none & $3.0 \mathrm{E}+08$ & $\begin{array}{l}\text { total alpha } \\
\text { total beta }\end{array}$ & $\begin{array}{l}2.0 \mathrm{E}-07 \\
3.5 \mathrm{E}-06\end{array}$ \\
\hline 296-S-16 (222-S Lab) & 3.0 & HEPA & $1.8 \mathrm{E}+06$ & $\begin{array}{l}\text { total alpha } \\
\text { total beta }\end{array}$ & $\begin{array}{l}1.7 E-09 \\
5.3 E-08\end{array}$ \\
\hline 296-S-21 (222-S Lab) & 11.6 & HEPA & $9.2 \mathrm{E}+08$ & $\begin{array}{l}\text { total alpha } \\
\text { total beta }\end{array}$ & $\begin{array}{c}\text { ND } \\
1.0 \mathrm{E}-06\end{array}$ \\
\hline 296-W-3 (Waste Compactor) & 7.6 & HEPA & $3.1 \mathrm{E}+07$ & \multicolumn{2}{|c|}{ ND } \\
\hline \multicolumn{6}{|c|}{300 Area Stacks } \\
\hline EP-3745-01-S (3745 Bldg.) & 2 & HEPA & $8.4 E+06$ & $\begin{array}{l}\text { total alpha } \\
\text { total beta }\end{array}$ & $\begin{array}{l}9.5 \mathrm{E}-09 \\
1.1 \mathrm{E}-07\end{array}$ \\
\hline $\begin{array}{l}\text { EP-3746A-01-V } \\
\text { (3746-A Bldg.) }\end{array}$ & 4 & HEPA & $5.5 \mathrm{E}+07$ & $\begin{array}{l}\text { total alpha } \\
\text { total beta }\end{array}$ & $\begin{array}{c}\text { ND } \\
5.1 \mathrm{E}-07\end{array}$ \\
\hline 377-SGEF (377 Bldg.) & 10 & HEPA & $6.2 E+06$ & $\begin{array}{l}\text { total alpha } \\
\text { total beta }\end{array}$ & $\begin{array}{l}8.1 E-09 \\
3.6 E-08\end{array}$ \\
\hline EP-303C-01-S (303-C Bldg.) & 4 & HEPA & $9.9 \mathrm{E}+06$ & $\begin{array}{l}\text { total alpha } \\
\text { total beta }\end{array}$ & $\begin{array}{l}4.6 \mathrm{E}-08 \\
1.6 \mathrm{E}-07\end{array}$ \\
\hline 306E-ULAB (306-E Bldg.) & 20.1 & HEPA & $4.4 E+07$ & $\begin{array}{l}\text { total alpha } \\
\text { total beta }\end{array}$ & $\begin{array}{l}4.2 \mathrm{E}-08 \\
1.8 \mathrm{E}-07\end{array}$ \\
\hline $\begin{array}{l}\text { EP-306W-01-V } \\
\text { (306-W Bldg.) }\end{array}$ & 8 & HEPA & $2.5 \mathrm{E}+08$ & $\begin{array}{l}\text { total alpha } \\
\text { total beta }\end{array}$ & $\begin{array}{l}1.6 E-07 \\
2.0 E-06\end{array}$ \\
\hline
\end{tabular}


Table 2-1. Hanford Site Radionuclide Air Emissions Data for 1992. (9 sheets)

\begin{tabular}{|c|c|c|c|c|c|}
\hline \multicolumn{6}{|c|}{ Minor Stack (has maximum dose potential of $<0.1 \mathrm{mrem} / \mathrm{yr}$ EDE to nearest offsite residence ${ }^{\mathrm{a}}$ ) } \\
\hline $\begin{array}{c}\text { ID }^{\mathrm{b}} \\
\text { (location) }\end{array}$ & $\begin{array}{l}\text { Discharge } \\
\text { Height (m) }\end{array}$ & $\begin{array}{l}\text { Emission } \\
\text { Controt }^{d}\end{array}$ & $\begin{array}{c}\text { Total Flow } \\
\left(\mathrm{m}^{3}\right)\end{array}$ & Radionuclide & Curies \\
\hline \multicolumn{6}{|c|}{300 Area Stacks (continued) } \\
\hline $\begin{array}{l}\text { EP-306W-02-V } \\
\text { (306-W Bldg.) }\end{array}$ & 8 & HEPA & $2.1 \mathrm{E}+08$ & $\begin{array}{l}\text { total alpha } \\
\text { total beta }\end{array}$ & $\begin{array}{c}\text { ND } \\
1.9 \mathrm{E}-06\end{array}$ \\
\hline $\begin{array}{l}\text { EP-306W-03-V } \\
\text { (306-W Bldg.) }\end{array}$ & 8 & HEPA & $3.2 \mathrm{E}+08$ & $\begin{array}{l}\text { total alpha } \\
\text { total beta }\end{array}$ & $\begin{array}{l}\text { ND } \\
\text { ND }\end{array}$ \\
\hline $\begin{array}{l}\text { EP-306W-04-S } \\
\text { (306-W Bldg.) }\end{array}$ & 10 & HEPA & $3.8 \mathrm{E}+08$ & $\begin{array}{l}\text { total alpha } \\
\text { total beta }\end{array}$ & $\begin{array}{c}\text { ND } \\
2.0 \mathrm{E}-06\end{array}$ \\
\hline 308-GL-EX (308 Bldg.) & 9.1 & HEPA & $4.9 \mathrm{E}+07$ & $\begin{array}{l}\text { total alpha } \\
\text { total beta }\end{array}$ & $\begin{array}{l}4.2 \mathrm{E}-08 \\
1.8 \mathrm{E}-07\end{array}$ \\
\hline 308-ET-EX (308 Bldg.) & 9.1 & HEPA & $4.7 E+07$ & $\begin{array}{l}\text { total alpha } \\
\text { total beta }\end{array}$ & $\begin{array}{l}9.2 E-08 \\
3.4 E-07\end{array}$ \\
\hline 308-TRIGA (308 Bldg.) & 11.3 & $\begin{array}{l}\text { HEPA, } \\
\text { charcoal }\end{array}$ & $3.0 \mathrm{E}+07$ & $\begin{array}{l}\text { total alpha } \\
\text { total beta }\end{array}$ & $\begin{array}{l}3.0 \mathrm{E}-08 \\
1.1 \mathrm{E}-07\end{array}$ \\
\hline EP-314-01-S (314 Bldg.) & 6 & HEPA & $4.3 E+07$ & $\begin{array}{l}\text { total alpha } \\
\text { total beta }\end{array}$ & $\begin{array}{l}3.0 \mathrm{E}-08 \\
4.2 \mathrm{E}-07\end{array}$ \\
\hline EP-318-01-S (318 Bldg.) & 9 & HEPA & $9.4 \mathrm{E}+07$ & $\begin{array}{l}\text { total alpha } \\
\text { total beta }\end{array}$ & $\begin{array}{ll}5.7 & E-08 \\
8.2 & E-07\end{array}$ \\
\hline EP-320-01-S (320 Bldg.) & 13 & HEPA & $5.7 \mathrm{E}+08$ & $\begin{array}{l}\text { total alpha } \\
\text { total beta }\end{array}$ & $\begin{array}{l}6.9 \mathrm{E}-07 \\
4.9 \mathrm{E}-06\end{array}$ \\
\hline EP-320-02-S (320 Bldg.) & 8 & HEPA & $7.8 \mathrm{E}+06$ & $\begin{array}{l}\text { total alpha } \\
\text { total beta }\end{array}$ & $\begin{array}{l}1.3 \mathrm{E}-08 \\
1.2 \mathrm{E}-07\end{array}$ \\
\hline EP-320-03-S (320 Bldg.) & 6 & HEPA & $1.3 E+07$ & $\begin{array}{l}\text { total alpha } \\
\text { total beta }\end{array}$ & $\begin{array}{c}\mathrm{ND} \\
1.1 \mathrm{E}-07\end{array}$ \\
\hline EP-320-04-S (320 Bldg.) & 6 & HEPA & $1.2 \mathrm{E}+07$ & $\begin{array}{l}\text { total alpha } \\
\text { total beta }\end{array}$ & $\begin{array}{l}9.4 \mathrm{E}-09 \\
1.3 \mathrm{E}-07\end{array}$ \\
\hline EP-323-01-S (323 Bldg.) & 5 & HEPA & $7.5 \mathrm{E}+07$ & $\begin{array}{l}\text { total alpha } \\
\text { total beta }\end{array}$ & $\begin{array}{l}7.8 \mathrm{E}-08 \\
1.1 \mathrm{E}-06\end{array}$ \\
\hline EP-326-01-S (326 Bldg.) & 14 & HEPA & $8.3 \mathrm{E}+08$ & $\begin{array}{l}{ }^{3} \mathrm{H} \text { (as HTO) } \\
{ }^{3} \mathrm{H}(\text { as HT) } \\
\text { total alpha } \\
\text { total beta }\end{array}$ & $\begin{array}{ll}1.9 & \mathrm{E}-01 \\
2.7 & \mathrm{E}-01 \\
1.1 & \mathrm{E}-06 \\
2.1 & \mathrm{E}-05\end{array}$ \\
\hline EP-329-01-V (329 Bldg.) & 11 & HEPA & $1.3 \mathrm{E}+08$ & $\begin{array}{l}\text { total alpha } \\
\text { total beta }\end{array}$ & $\begin{array}{c}\text { ND } \\
1.3 \mathrm{E}-06\end{array}$ \\
\hline EP-329-02-V (329 Bldg.) & 10 & HEPA & $2.0 \mathrm{E}+08$ & $\begin{array}{l}\text { total alpha } \\
\text { total beta }\end{array}$ & $\begin{array}{c}\text { ND } \\
7.9 \mathrm{E}-07\end{array}$ \\
\hline EP-329-03-V (329 Bldg.) & 10 & HEPA & $1.6 E+08$ & $\begin{array}{l}\text { total alpha } \\
\text { total beta }\end{array}$ & $\begin{array}{l}1.2 E-07 \\
3.5 E-06\end{array}$ \\
\hline
\end{tabular}


Table 2-1. Hanford Site Radionuclide Air Emissions Data for 1992. (9 sheets) Minor Stack (has maximum dose potential of $<0.1$ mrem/yr EDE to nearest offsite residence ${ }^{\mathrm{a}}$ )

\begin{tabular}{|c|c|c|c|c|c|}
\hline $\begin{array}{c}\text { ID }^{\mathbf{b}} \\
\text { (beation) }\end{array}$ & $\begin{array}{c}\text { Discharge } \\
\text { Height (m) }\end{array}$ & $\begin{array}{l}\text { Emission } \\
\text { Control }^{\text {d }}\end{array}$ & $\begin{array}{c}\text { Total Flow } \\
\left(\mathrm{m}^{3}\right)\end{array}$ & Radionuclide & Curies \\
\hline \multicolumn{6}{|c|}{300 Area Stacks (continued) } \\
\hline EP-329-04-V (329 Bldg.) & 5 & HEPA & $3.0 \mathrm{E}+07$ & $\begin{array}{l}\text { total alpha } \\
\text { total beta }\end{array}$ & $\begin{array}{c}\text { ND } \\
2.7 \mathrm{E}-07\end{array}$ \\
\hline $\begin{array}{l}\text { EP-331-01-V (formerly } \\
\text { associated with "D1") } \\
\text { (331 Bldg.) }\end{array}$ & 15 & HEPA & $9.7 \mathrm{E}+08$ & $\begin{array}{l}\text { total alpha } \\
\text { total beta }\end{array}$ & $\begin{array}{c}\mathrm{ND} \\
6.6 \mathrm{E}-06\end{array}$ \\
\hline $\begin{array}{l}\text { EP-331-01-V (D2) } \\
\text { (331 Bldg.) }\end{array}$ & \multicolumn{5}{|c|}{$\begin{array}{l}\text { (not an actual emission point but a sampling point instead; } \\
\text { will be deregistered with WDOH) }\end{array}$} \\
\hline $\begin{array}{l}\text { EP-331A-01-S } \\
(331-A \text { Bldg.) }\end{array}$ & 5 & HEPA & $9.9 \mathrm{E}+07$ & $\begin{array}{l}\text { total alpha } \\
\text { total beta }\end{array}$ & $\begin{array}{c}\mathrm{ND} \\
6.4 \mathrm{E}-07\end{array}$ \\
\hline EP-331A-02-V (331 Bldg.) & 6 & HEPA & $8.0 E+06$ & $\begin{array}{l}\text { total alpha } \\
\text { total beta }\end{array}$ & $\begin{array}{c}\text { ND } \\
1.0 \mathrm{E}-07\end{array}$ \\
\hline $\begin{array}{l}\text { EP-331H-01-S } \\
\text { (331-H Bldg.) }\end{array}$ & 6 & HEPA & $8.2 E+07$ & $\begin{array}{l}\text { total alpha } \\
\text { total beta }\end{array}$ & $\begin{array}{l}\text { ND } \\
6.3 \mathrm{E}-07\end{array}$ \\
\hline EP-3708-01-S (3708 Bldg.) & 7 & HEPA & $9.6 \mathrm{E}+07$ & $\begin{array}{l}\text { total alpha } \\
\text { total beta }\end{array}$ & $\begin{array}{l}\mathrm{ND} \\
8.0 \mathrm{E}-07\end{array}$ \\
\hline EP-3720-02-S (3720 Bldg.) & 5 & HEPA & $3.9 \mathrm{E}+07$ & $\begin{array}{l}\text { total alpha } \\
\text { total beta }\end{array}$ & $\begin{array}{l}4.8 \mathrm{E}-08 \\
4.3 \mathrm{E}-07\end{array}$ \\
\hline $\begin{array}{l}\text { EP-3720-03-S } \\
\text { (3720 Bldg.) }\end{array}$ & 9 & HEPA & $8.0 \mathrm{E}+07$ & $\begin{array}{l}\text { total alpha } \\
\text { total beta }\end{array}$ & $\begin{array}{l}\text { ND } \\
5.7 \mathrm{E}-07\end{array}$ \\
\hline EP-3730-01-S (3730 Bldg.) & 5 & HEPA & $6.2 \mathrm{E}+06$ & $\begin{array}{l}\text { total alpha } \\
\text { total beta }\end{array}$ & $\begin{array}{c}\text { ND } \\
3.4 \mathrm{E}-08\end{array}$ \\
\hline \multicolumn{6}{|c|}{400 Aren Stacks } \\
\hline FFTF-CB-EX (FFTF) & 14.3 & none & $3.6 \mathrm{E}+08$ & $\begin{array}{l}{ }^{41} \mathrm{Ar} \\
\text { total alpha } \\
\text { total beta }\end{array}$ & $\begin{array}{ll}8.5 & E+00 \\
1.1 & E-06 \\
6.7 & E-06\end{array}$ \\
\hline FFTF-RE-SB (FFTF) & 6.1 & none & $1.9 \mathrm{E}+08$ & $\begin{array}{l}\text { total alpha } \\
\text { total beta }\end{array}$ & $\begin{array}{l}7.8 E-07 \\
5.2 E-06\end{array}$ \\
\hline FFTF-HT-TR (FFTF) & 8.8 & none & $6.2 \mathrm{E}+07$ & $\begin{array}{l}\text { total alpha } \\
\text { total beta }\end{array}$ & $\begin{array}{l}7.5 \mathrm{E}-08 \\
4.6 \mathrm{E}-07\end{array}$ \\
\hline 437-MN\&ST (MASF) & 9.1 & HEPA & $2.4 \mathrm{E}+08$ & $\begin{array}{l}\text { total alpha } \\
\text { total beta }\end{array}$ & $\begin{array}{l}1.9 \mathrm{E}-07 \\
7.4 \mathrm{E}-07\end{array}$ \\
\hline
\end{tabular}


Table 2-1. Hanford Site Radionuclide Air Emissions Data for 1992. (9 sheets)

\begin{tabular}{|c|c|c|c|c|c|}
\hline \multicolumn{6}{|c|}{ Minor Stack (has maximum dose potential of $<0.1 \mathrm{mrem} / \mathrm{yr}$ EDE to nearest of fsite residence ${ }^{3}$ ) } \\
\hline $\begin{array}{c}\text { ID }^{b} \\
\text { (location) }\end{array}$ & $\begin{array}{l}\text { Discharge } \\
\text { Height (m) }\end{array}$ & $\begin{array}{l}\text { Emission } \\
\text { Control }^{d}\end{array}$ & $\begin{array}{c}\text { Total Flow } \\
\left(\mathrm{m}^{3}\right)\end{array}$ & Radionuclide & Curies \\
\hline \multicolumn{6}{|c|}{600 Area Stacks } \\
\hline $\begin{array}{l}\text { EP-6652H-01-V } \\
(6652-H \text { Bldg.) }\end{array}$ & 3.6 & HEPA & $5.3 \mathrm{E}+07$ & $\begin{array}{l}\text { total alpha } \\
\text { total beta }\end{array}$ & $\begin{array}{l}2.9 \mathrm{E}-08 \\
5.0 \mathrm{E}-07\end{array}$ \\
\hline $\begin{array}{l}\text { EP-6652H-02-V } \\
(6652-\mathrm{H} \text { Bldg. })\end{array}$ & \multicolumn{5}{|c|}{$\begin{array}{c}\text { (not an actual emission point but a sampling point instead; } \\
\text { will be deregistered with WDOH) }\end{array}$} \\
\hline $\begin{array}{l}\text { EP-ISV-G-01-Th; not } \\
\text { registered with WDOH }\end{array}$ & 2 & HEPA & $\mathrm{i}$ & \multicolumn{2}{|c|}{ ND } \\
\hline
\end{tabular}

'Determining the state of NESHAP Subpart $H$ compliance for each emission point involved using nearest offsite residences, which differed from the MEI.

${ }^{b} \mathrm{D}=$ Identification, i.e., the alpha-numeric designator for the respective stack or vent.

${ }^{\mathrm{c}}$ Nearest residence $=\mathrm{A}$ residence closest to the specific area considered, as opposed to the entire Site, which is represented by the MEI location of Ringold, Washington.

Efficiencies are: $99.95 \%$ for HEPA; $95 \%$ for charcoal; $99.8 \%$ for sand filter; $0 \%$ for no emission control.

${ }^{e} E D E=$ Effective dose equivalent for the MEI at Ringold, Washington, not the nearest residence.

${ }^{\mathrm{N}} \mathrm{ND}=$ none detected, i.e., the average of all the measurements for that given radionuclide or type of radioactivity made during the year was below background levels.

${ }^{8} \mathrm{HTO}$ is tritium as condensable water vapor; $\mathrm{HT}$ is tritium as noncondensable gas.

${ }^{b}$ Greenhouse at In Situ Yitrification site; operated from August 12 through 14, 1992.

This greenhouse operated for only two days with emissions below detection limits; therefore, no annual exhaust flow was calculated. 
Table 2-2. Distances to the Nearest Offsite Residences from Hanford Site Areas.

\begin{tabular}{|l|l|}
\hline Area & \multicolumn{1}{|c|}{$\begin{array}{c}\text { Nearest Offsite } \\
\text { Residence }\end{array}$} \\
\hline 100 Areas & $6.1 \mathrm{mi}(9.9 \mathrm{~km})$ west \\
\hline 200 East Area & $9.9 \mathrm{mi}(16 \mathrm{~km})$ east \\
\hline 200 West Area & $15 \mathrm{mi}(24 \mathrm{~km})$ east \\
\hline 300 Area & $\begin{array}{l}0.87 \mathrm{mi}(1.4 \mathrm{~km}) \text { northeast*; } \\
0.62 \mathrm{mi}(1.0 \mathrm{~km}) \text { south** }\end{array}$ \\
\hline 400 Area & $5.0 \mathrm{mi}(8.1 \mathrm{~km})$ south \\
\hline 600 Area & $1.5 \mathrm{mi}(2.4 \mathrm{~km})$ south- \\
& southeast \\
\hline
\end{tabular}

*Westinghouse Hanford stacks

**PNL stacks 


\subsection{POINT-SOURCE EMISSION DOSE ASSESSMENTS}

\subsection{DESCRIPTION OF POINT-SOURCE EMISSION DOSE MODEL}

The CAP-88 computer code (Beres 1990) was used to demonstrate compliance with the $10 \mathrm{mrem} / \mathrm{yr}$ EDE standard contained in 40 CFR 61.92 (EPA 1992). Because the Hanford Site has numerous widely separated points sources of emissions, it was necessary to determine the location at which the maximum dose would be received from combined air emissions originating from all point sources. The emission points used for modeling purposes were centered on each of the major Hanford Site operating areas that released radionuclides to the atmosphere during 1992. For the 200 Areas, the reference point was the meteorology tower approximately midway between the 200 East and 200 West Areas. A farm at Ringold, Washington, was determined to be the best location to use in the compliance analysis. The farm is directly across the Columbia River from the Hanford Site boundary to the east-southeast of the 200 Areas. The major operating areas and their location with respect to the MEI at Ringold are listed in Table 3-1. The table also lists the dose to this receptor from a $1-\mathrm{Ci}$ release of specific radionuclides at each location. The actual stack releases from each operating area are listed in Table 3-2. Combined doses to the receptor from each of the areas were calculated from the unit dose factors and the stack releases for the compliance assessment. The distribution of dose to this individual by radionuclide from each release point is presented in Table 3-3.

\subsection{SUMMARY OF INPUT PARAMETERS}

Dose calculations were performed using established standard parameters for the Hanford Site and its environment (Woodruff and Hanf 1992). Releases from stacks were modeled using an effective stack height as shown in Table 3-1. The dose from elemental tritium (HT) was estimated by assuming that it contributes approximately $1 \%$ of the dose from a comparable release of tritiated water vapor (HTO) as a result of oxidation in the environment (Brown 1989). This was necessary because CAP- 88 uses a specific-activity model based on tritium as water vapor, which overestimates the dose from elemental tritium by several orders of magnitude. Releases of ${ }^{220} \mathrm{Rn}$ were modeled by assuming that this shortlived gas had decayed to the next long-lived member of its decay chain, ${ }^{212} \mathrm{~Pb}$, before transport offsite. Releases reported as total alpha or total beta were evaluated as ${ }^{239} \mathrm{Pu}$ or ${ }^{90} \mathrm{Sr}$, respectively, to conservatively estimate their expected impact.

A combination of actual radionuclide data and default values from the CAP-88 data libraries for radionuclide-specific parameters were used in the dose calculations. Parameters used for the ingestion pathway assumed that the receptor's entire diet was produced at the MEI residence in Ringold and that maximum individual exposure and consumption parameters were those determined previously for the Hanford Site. 
Table 3-1. Release Estimates of Hanford Site

Radionuclide Air Emissions in 1992.

\begin{tabular}{|c|c|c|c|c|c|}
\hline Release Location - & 100 Areas & 200 Areas & 300 Area & 400 Area & Total \\
\hline Release Height - & $89 \mathrm{~m}$ & $89 \mathrm{~m}$ & $10 \mathrm{~m} / 61 \mathrm{~m}$ & $10 \mathrm{~m}$ & \\
\hline Radionuclide $\nabla$ & \multicolumn{5}{|c|}{$(\mathbf{C i})$} \\
\hline${ }^{3} \mathrm{H}$ & & $\cdot$ & $2.6 \mathrm{E}+01 / 9.6 \mathrm{E}+00^{2}$ & & $3.5 \mathrm{E}+01^{4}$ \\
\hline${ }^{41} \mathrm{Ar}$ & & & & $8.5 E+00$ & $8.5 \mathrm{E}+\infty 0$ \\
\hline${ }^{\infty} \mathrm{Co}$ & $3.8 \mathrm{E}-06$ & & & & $3.8 E-06$ \\
\hline${ }^{10} \mathrm{Sr}$ & $1.6 E-04$ & $4.4 \mathrm{E}-04^{\mathrm{b}}$ & $5.7 \mathrm{E}-05 / 6.4 \mathrm{E}-07^{6}$ & & $6.6 \mathrm{E}-04^{b}$ \\
\hline${ }^{106} \mathrm{Ru}$ & $3.7 \mathrm{E}-05$ & & & & $3.7 \mathrm{E}-05$ \\
\hline${ }^{122} \mathrm{Sb}$ & $1.1 \mathrm{E}-05$ & & & & $1.1 \mathrm{E}-05$ \\
\hline 129 & & $3.0 \mathrm{E}-02$ & & & $3.0 \mathrm{E}-02$ \\
\hline${ }^{137} \mathrm{Cs}$ & $2.6 \mathrm{E}-04$ & $2.3 \mathrm{E}-03$ & & $1.3 \mathrm{E}-05$ & $2.6 \mathrm{E}-03$ \\
\hline${ }^{147} \mathrm{Pm}$ & & $1.7 \mathrm{E}-04$ & & & $1.7 \mathrm{E}-04$ \\
\hline${ }^{154} \mathrm{Eu}$ & $5.4 \mathrm{E}-06$ & & & & $5.4 \mathrm{E}-06$ \\
\hline${ }^{212} \mathrm{~Pb}$ & & $5.2 \mathrm{E}-02^{\mathrm{c}}$ & & & $5.2 \mathrm{E}-02^{\mathrm{c}}$ \\
\hline $200 \mathrm{Rn}$ & & $3.4 \mathrm{E}+01$ & & & $3.4 \mathrm{E}+01$ \\
\hline $234 \mathrm{U}$ & & $5.2 \mathrm{E}-07$ & $2.1 E-08$ & & $5.4 \mathrm{E}-07$ \\
\hline$\overline{235} \mathrm{U}$ & & $1.8 \mathrm{E}-08$ & $1.1 \mathrm{~F}-09$ & & $1.9 \mathrm{E}-08$ \\
\hline${ }^{236} \mathrm{U}$ & & $3.5 \mathrm{E}-08$ & & & $3.5 \mathrm{E}-08$ \\
\hline 230 & & $3.5 \mathrm{E}-07$ & $2.0 \mathrm{E}-08$ & & $3.7 \mathrm{E}-07$ \\
\hline${ }^{238} \mathrm{Pu}$ & $1.3 \mathrm{E}-06$ & $1.3 \mathrm{E}-05$ & & & $1.4 \mathrm{E}-05$ \\
\hline $299,200 \mathrm{Pu}$ & $8.7 \mathrm{E}-06$ & $4.7 E-04^{d}$ & $3.9 \mathrm{E}-06 / 3.9 \mathrm{E}-07$ & & $4.9 \mathrm{E}-04$ \\
\hline${ }^{2 n 1} \mathrm{Pu}$ & $3.9 \mathrm{E}-05$ & $3.4 \mathrm{E}-03$ & & & $3.4 \mathrm{E}-03$ \\
\hline${ }^{211} \mathrm{Am}$ & $5.1 \mathrm{E}-06$ & $2.1 \mathrm{E}-04$ & & & $2.2 E-04$ \\
\hline
\end{tabular}

The $35 \mathrm{Ci}$ total reflects about $34.8 \mathrm{Ci}$ of $\mathrm{HTO}$ and about $9.5 \mathrm{Ci}$ of HT. The release of one $\mathrm{Ci}$ of $\mathrm{HT}$ to the air, however, is equivalent to the dise effect from the release of about $0.01 \mathrm{Ci}$ of HTO to the air (i.e., 0.095 $\mathrm{Ci} \mathrm{HTO}$ ); hence, the $35 \mathrm{Ci}$ total.

${ }^{b}$ This value represents bols ${ }^{\circ} \mathrm{Sr}$ and total beta release data. For dose calculations, total beta data are construed conservatively as ${ }^{90} \mathrm{Sr}$ for those stacks whose emissions are not analyzed for this radionuclide.

Includes release of $2.7 \mathrm{E}-03 \mathrm{Ci}$ of ${ }^{212} \mathrm{~Pb}$ in addition to ingrowth from release of $34 \mathrm{Ci}$ of ${ }^{20} \mathrm{Rn}$.

This value represents both 299,2102 , and total a!pha releases. For dose calculations, total alpha data are construed conservatively as $29.200 \mathrm{Pu}$ for stacks whose emissions are not analyzed for this radionuclide. 
Table 3-2. CAP-88 Unit Dose Factors for the Offsite Hypothetical Maximally Exposed Individual Affected by Radionuclide Air Emissions from the Hanford Site in 1992.

\begin{tabular}{|c|c|c|c|c|}
\hline Release Location & 100 Areas & 200 Areas & 300 Area & 400 Area \\
\hline Release Height $>$ & $89 \mathrm{~m}$ & $89 \mathrm{~m}$ & $10 \mathrm{~m} / 61 \mathrm{~m}$ & $10 \mathrm{~m}$ \\
\hline $\begin{array}{l}\text { MEI' } \\
\text { Location - }\end{array}$ & $\begin{array}{c}19 \mathrm{mi} \\
(30 \mathrm{~km}) \mathrm{SE}\end{array}$ & $\begin{array}{c}16 \mathrm{mi} \\
(26 \mathrm{~km}) \mathrm{ESE}\end{array}$ & $\begin{array}{c}8.1 \mathrm{mi} \\
(13 \mathrm{~km}) \mathrm{N}\end{array}$ & $\begin{array}{c}6,8 \mathrm{mi} \\
(11 \mathrm{~km}) \mathrm{NE}\end{array}$ \\
\hline Radionuclide & \multicolumn{4}{|c|}{ Effective Dose Equivalent (mrem/Ci-yr) } \\
\hline${ }^{3} \mathrm{H}$ & & & $2.66 \mathrm{E}-05 / 8.49 \mathrm{E}-06$ & \\
\hline${ }^{41} \mathrm{Ar}$ & & & & $9.06 \mathrm{E}-06$ \\
\hline${ }^{60} \mathrm{Co}$ & $2.38 \mathrm{E}-03$ & & & \\
\hline${ }^{90} \mathrm{Sr}$ & $3.59 \mathrm{E}-03$ & $4.60 \mathrm{E}-03$ & $5.00 \mathrm{E}-02 / 1.85 \mathrm{E}-02$ & \\
\hline${ }^{106} \mathrm{Ru}$ & $1.44 \mathrm{E}-03$ & & & \\
\hline${ }^{125} \mathrm{Sb}$ & $3.41 \mathrm{E}-04$ & & & \\
\hline$\sqrt{129} \mathrm{I}$ & & $6.74 E-02$ & & \\
\hline${ }^{137} \mathrm{Cs}$ & $1.17 \mathrm{E}-03$ & $1.50 \mathrm{E}-03$ & & $1.05 \mathrm{E}-02$ \\
\hline${ }^{147} \mathrm{Pm}$ & & $1.19 \mathrm{E}-04$ & & \\
\hline${ }^{154} \mathrm{Eu}$ & $1.50 \mathrm{E}-03$ & & & \\
\hline${ }^{212} \mathrm{~Pb}$ & & $3.63 \mathrm{E}-04^{\mathrm{b}}$ & & \\
\hline${ }^{234} \mathrm{U}$ & & $3.35 \mathrm{E}-01$ & $3.65 \mathrm{E}+00 / 1.35 \mathrm{E}+00$ & \\
\hline${ }^{225} \mathrm{U}$ & & $3.11 \mathrm{E}-01$ & $3.39 \mathrm{E}+00 / 1.25 \mathrm{E}+00$ & \\
\hline $206 \mathrm{U}$ & & $3.17 \overline{E-01}$ & & \\
\hline${ }^{238} \mathrm{U}$ & & $2.99 \mathrm{E}-01$ & $3.25 \mathrm{E}+00 / 1.20 \mathrm{E}+00$ & \\
\hline${ }^{228} \mathrm{Pu}$ & $6.59 \mathrm{E}-01$ & $8.43 \mathrm{E}-01$ & & \\
\hline $239,240 \mathrm{Pu}$ & $7.12 \mathrm{E}-01$ & $9.11 \mathrm{E}-01$ & $9.91 \mathrm{E}+00 / 3.67 \mathrm{E}+00$ & $6.34 \mathrm{E}+00$ \\
\hline${ }^{2+1} \mathrm{Pu}$ & $1.13 \mathrm{E}-02$ & $1.45 \mathrm{E}-02$ & & \\
\hline${ }^{241} \mathrm{Am}$ & $1.08 \mathrm{E}+00$ & $1.38 \mathrm{E}+00$ & & \\
\hline
\end{tabular}

${ }^{2} \mathrm{MEI}=$ Maximally exposed individual.

bDose for this radionuclide is the result of ingrowth from the parent. 
Table 3-3. CAP-88 Dose Estimates for the Offsite Hypothetical Maximally Exposed Individual Affected by Radionuclide Air Emissions from the Hanford Site in 1992.

\begin{tabular}{|c|c|c|c|c|c|c|c|c|c|c|}
\hline Release & 100 & eas & $200 A$ & eas & $300 A$ & rea & $400 \mathrm{~A}$ & rea & Site 1 & \\
\hline Nuclider & $\begin{array}{c}\text { Dose } \\
\text { (mrem) }\end{array}$ & $\begin{array}{c}\text { \% Area } \\
\text { Total }\end{array}$ & $\begin{array}{c}\text { Dose } \\
\text { (mrem) }\end{array}$ & $\begin{array}{c}\text { \% Area } \\
\text { Total }\end{array}$ & $\begin{array}{c}\text { Dose } \\
\text { (mrem) }\end{array}$ & $\begin{array}{c}\text { \% Area } \\
\text { Total }\end{array}$ & $\begin{array}{c}\text { Dose } \\
\text { (mrem) }\end{array}$ & $\begin{array}{c}\% \text { Area } \\
\text { Total }\end{array}$ & $\begin{array}{c}\text { Dose } \\
\text { (mrem) }\end{array}$ & $\%$ \\
\hline${ }^{3} \mathrm{H}$ & & & & & $7.6 \mathrm{E}-04$ & 95 & & & 7.6 E-04 & 20 \\
\hline${ }^{41} \mathrm{Ar}$ & & & & & & & 7.7 E-05 & 85 & $7.7 \mathrm{E}-05$ & 2.1 \\
\hline${ }^{60} \mathrm{Co}$ & $9.0 \mathrm{E}-09$ & 0.06 & & & & & & & 9.0 E-09 & 0 \\
\hline${ }^{90} \mathrm{Sr}^{2}$ & 6.1 E-07 & 4.4 & $2.2 \mathrm{E}-06$ & 0.08 & $3.0 \mathrm{E}-06$ & 0.38 & & & $5.8 \mathrm{E}-06$ & 0.15 \\
\hline${ }^{106} \mathrm{Ru}$ & 5.9 E-08 & 0.4 & & & & & & & $5.9 \mathrm{E}-08$ & 0 \\
\hline${ }^{125} \mathrm{Sb}$ & 4.0 E-09 & 0.03 & & & & & & & 4.0 E-09 & 0 \\
\hline${ }^{129} 1$ & & & $2.0 \mathrm{E}-03$ & 71 & & & & & $2.0 \mathrm{E}-03$ & 54 \\
\hline${ }^{137} \mathrm{Cs}$ & 4.1 E-07 & 2.9 & 4.6 E-06 & 0.16 & & & $1.8 \mathrm{E}-07$ & 0.2 & 5.2 E-06 & 0.14 \\
\hline${ }^{147} \mathrm{Pm}$ & & & $2.0 \mathrm{E}-08$ & 0 & & & & & $2.0 \mathrm{E}-08$ & 0 \\
\hline${ }^{154} \mathrm{Eu}$ & $8.1 \mathrm{E}-09$ & 0.06 & & & & & & & 8.1 E-09 & 0 \\
\hline${ }^{212} \mathrm{~Pb}$ & & & $2.0 \mathrm{E}-05$ & 0.71 & & & & & $2.0 \mathrm{E}-05$ & 0.54 \\
\hline${ }^{234} U$ & & & 1.7 E-07 & 0.01 & 7.7 E-08 & 0.01 & & & 2.5 E-07 & 0.01 \\
\hline${ }^{235} \mathrm{U}$ & & & 5.6 E-09 & 0 & 3.7 E-09 & 0 & & & 9.3 E-09 & 0 \\
\hline${ }^{236} \mathrm{U}$ & & & $1.1 \mathrm{E}-08$ & 0 & & & & & 1.1 E-08 & 0 \\
\hline${ }^{236} \mathrm{U}$ & & & $1.1 \mathrm{E}-07$ & 0 & $6.5 \mathrm{E}-08$ & 0.01 & & & 1.7 E-07 & 0 \\
\hline${ }^{238} \mathrm{Pu}$ & 8.6 E-07 & 6.1 & $1.1 \mathrm{E}-05$ & 0.39 & & & & & 1.2 E-05 & 0.32 \\
\hline $239.210 \mathrm{Pu}^{\mathrm{b}}$ & 6.2 E-06 & 44 & $4.3 \mathrm{E}-04$ & 15.4 & $4.0 \mathrm{E}-05$ & 5.0 & 1.3 E-05 & 14.3 & $4.9 \mathrm{E}-04$ & 13 \\
\hline${ }^{2 A 1} \mathrm{Pu}$ & 4.4 E-07 & 3.1 & $4.9 \mathrm{E}-05$ & 1.8 & & & & & 5.0 E-05 & 1.3 \\
\hline${ }^{241} \mathrm{Am}$ & $5.5 \mathrm{E}-06$ & 39 & 2.9 E-04 & 10.4 & & & & & $3.0 \mathrm{E}-04$ & 7.9 \\
\hline $\begin{array}{l}\text { Area } \\
\text { Total }\end{array}$ & 1.4 E-05 & & 2.8 E-03 & & $8.0 \mathrm{E}-04$ & & 9.1 E-05 & & $3.7 \mathrm{E}-03$ & \\
\hline $\begin{array}{l}\% \text { Site } \\
\text { Total }\end{array}$ & 0.38 & & 75.71 & & 21.5 & & 2.42 & & 100 & \\
\hline
\end{tabular}

"Values represent both total beta and ${ }^{90} \mathrm{Sr}$ release data. For dose calculations, the total beta data are construed conservatively as ${ }^{90} \mathrm{Sr}$ for those stacks whose emissions are not analyzed for this radionuclide.

balues represent both total alpha and ${ }^{239,210} \mathrm{Pu}$ release data. For dose calculations, the total alpha data are construed conservatively as ${ }^{239.200} \mathrm{Pu}$ for those stacks whose emissions are not analyzed for this radionuclide. 
Radionuclide air concentrations at the MEI location were determined using meteorological data specific to each release point. Joint frequency distributions and CAP-88 wind files were prepared from data collected at the network of weather stations positioned throughout the Hanford Site operating areas, and these distributions represent the average of hourly data taken during 1992. This information was used to determine annual average dispersion coefficients for each collective emission point representing all the other emission points in the respective operating area.

\subsection{COMPLIANCE ASSESSMENT}

The combined dose to a receptor at the Ringold farm from air emissions at all Hanford Site operating areas during 1992 was 0.0037 mrem EDE, which is less than $0.1 \%$ of the $10 \mathrm{mrem} / \mathrm{yr}$ standard. As shown in Table 3-3, this dose resulted mainly from emissions related to residual radioactive material at facilities in the 200 Areas that are currently not operating. Operations at the 300 Area accounted for approximately $20 \%$ of the offsite dose. Contributions from other facilities were negligible. The radionuclides that contributed more than $10 \%$ of the total dose were tritium $(20.33 \%)$, ${ }^{129} \mathrm{I}(54.14 \%)$, and ${ }^{239,240} \mathrm{Pu}(13.06 \%)$.

The maximally exposed individual used for the Hanford Site compliance calculations is located approximately $16 \mathrm{mi}(26 \mathrm{~km})$ east-south-east of facilities in the 200 Areas, based on a hypothetical release point at the Hanford Meteorology Station. This location was chosen to represent emissions from the 200 Areas because it lies between two major groups of facilities that account for the bulk of stack releases. An investigation of the effect of this modeling convention on the overall dose indicated that it has a relatively minor impact. Even if all releases from the 200 Areas were assumed to originate at the facility nearest the Ringold receptor, their contribution to the total dose would increase by less than $30 \%$.

The percent each radionuclide contributed to the area and Hanford Site totals is shown in Table 3-3. Dose estimates and portions of the Hanford Site total dose attributable to each major stack and all the minor stacks and vents are shown in Table 3-4.

Again, for emphasis:

\section{HANFORD SITE EDE FOR 1992: 0.0037 mrem}

This dose is less than annual doses of the previous two years. The 40 CFR 61 requirement for annually reporting radionuclide air emissions from DOE facilities first applied to 1990 emissions (EPA 1992). Below are the offsite MEI doses attributable to Hanford Site emissions for the years 1990 and 1991.

- $\quad 0.0093$ mrem EDE, for 1990 (DOE-RL 1991)

- $\quad 0.0068$ mrem EDE, for 1991 (DOE-RL 1992). 
Table 3-4. Total Doses from Hanford Site Radionuclide Air Emissions in 1992.

\begin{tabular}{|c|c|c|}
\hline $\begin{array}{l}\text { Hanford Site } \\
\text { Maje- Stacks }\end{array}$ & $\begin{array}{c}\text { Dose } \\
\text { (mrem EDE })\end{array}$ & $\begin{array}{l}\% \text { of Hanford Site- } \\
\text { Related Offsite Dose }\end{array}$ \\
\hline 291-A-1 & $2.0 \mathrm{E}-03$ & 55 \\
\hline 291-Z-1 & $5.3 \mathrm{E}-04$ & 14 \\
\hline 291-B-1 & $3.7 \mathrm{E}-06$ & 0.1 \\
\hline $291-\mathrm{T}-1$ & $2.3 \mathrm{E}-05$ & 0.62 \\
\hline $291-A-22$ & $9.3 \mathrm{E}-09$ & 0.0 \\
\hline $296-\mathrm{A}-40$ & $1.5 \mathrm{E}-10$ & 0.0 \\
\hline 340-NT-EX & $2.1 \mathrm{E}-07$ & 0.0 \\
\hline EP-324-01-S & $8.3 \mathrm{E}-05$ & 2.2 \\
\hline EP-325-01-S & $6.8 \mathrm{E}-04$ & 18 \\
\hline EP-327-01-S & $1.1 \mathrm{E}-06$ & 0.03 \\
\hline EP-327-02-V & $1.9 \mathrm{E}-08$ & 0.0 \\
\hline EP-3720-01-S & $5.0 \mathrm{E}-07$ & 0.0 \\
\hline Major Stack Totals & $3.36 \mathrm{E}-03$ & 90 \\
\hline Minor Stack Totals & $3.7 \mathrm{E}-04$ & 10 \\
\hline ALL STACK TOTALS & $3.73 \mathrm{E}-03$ & 100 \\
\hline
\end{tabular}

${ }^{2} \mathrm{E}$. $\mathrm{E}=\mathrm{Efreclive}$ dose equivalent. 


\subsection{METEOROLOGICAL DATA}

Radionuclide air emissions disperse once they enter the atmosphere. Atmospheric dispersion models predict the degree of dilution and the magnitude of resulting air concentrations. Sitespecific measurements of the occurrence frequencies for wind speed, wind direction, and atmospheric stability are used in the models. The dispersion models yield annual average dispersion factors (in units of $\mathrm{s} / \mathrm{m}^{3}$ ). Combining these factors with annual average release rates predicts average radionuclide air concentrations for the year. Annual average dispersion factors around the 100, 200, 300, and 400 Areas for 1992 are in Tables 3-5 through 3-9. Population exposure to radionuclide air emissions was determined using values of population-weighted atmospheric dispersion factors for distance and each compass sector.

\subsection{UNPLANNED RELEASES OF RADIONUCLIDES TO THE ATMOSPHERE}

During 1992, no unplanned releases of radionuclides to the atmosphere from facilities at the Hanford Site occurred. Releases from point sources on the Hanford Site were monitored. The resulting quantities of radionuclides released and the corresponding effective dose equivalents are reported in Sections 2.0 and 3.0 of this document.

\subsection{ADDITIONAL INFORMATION}

\subsubsection{Projects Requiring Approval or Waiver Under 40 CFR 61}

In 1992, no projects were completed that needed approval for construction or waiver for modifications under 40 CFR 61 (EPA 1992). 


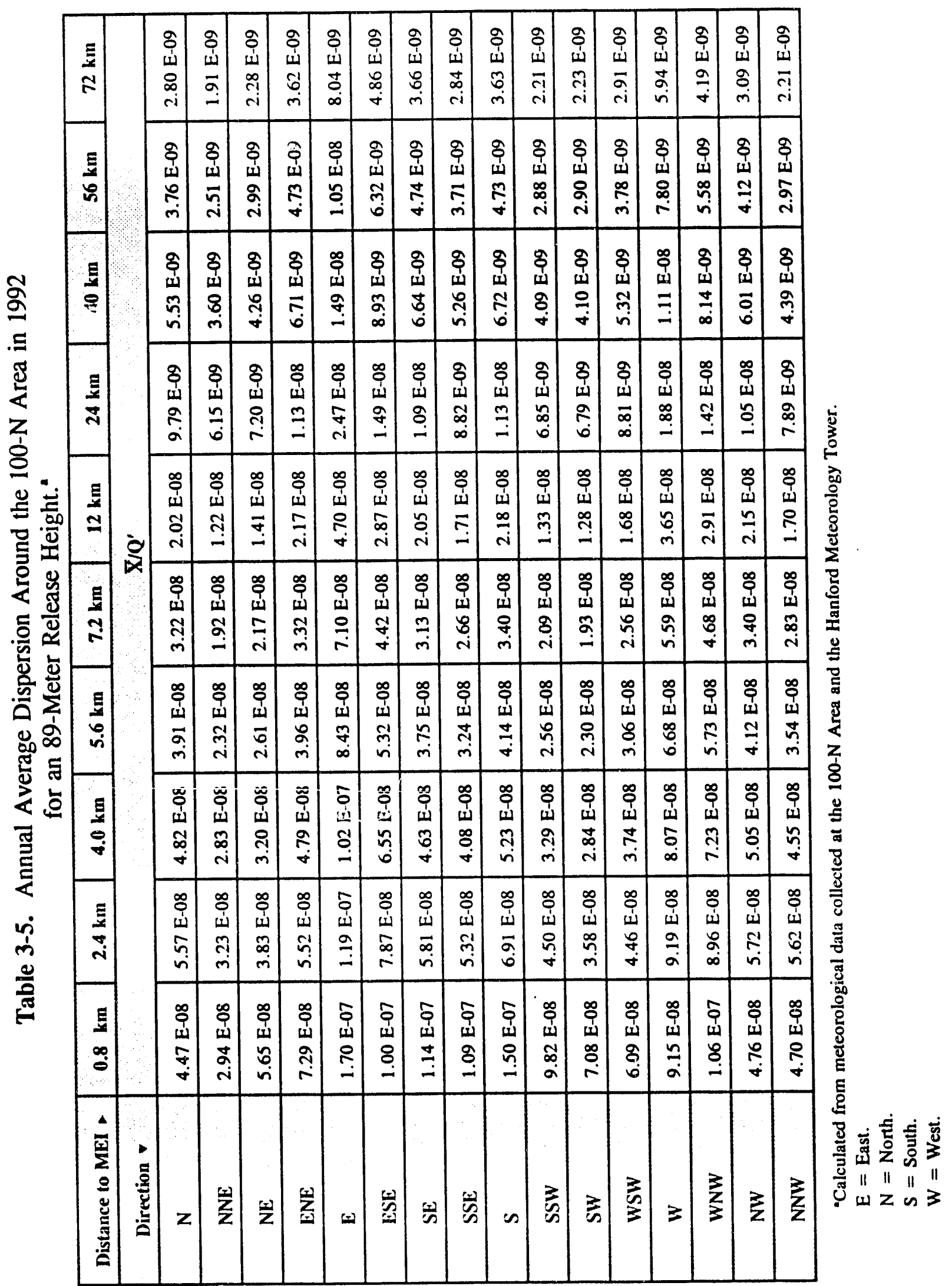




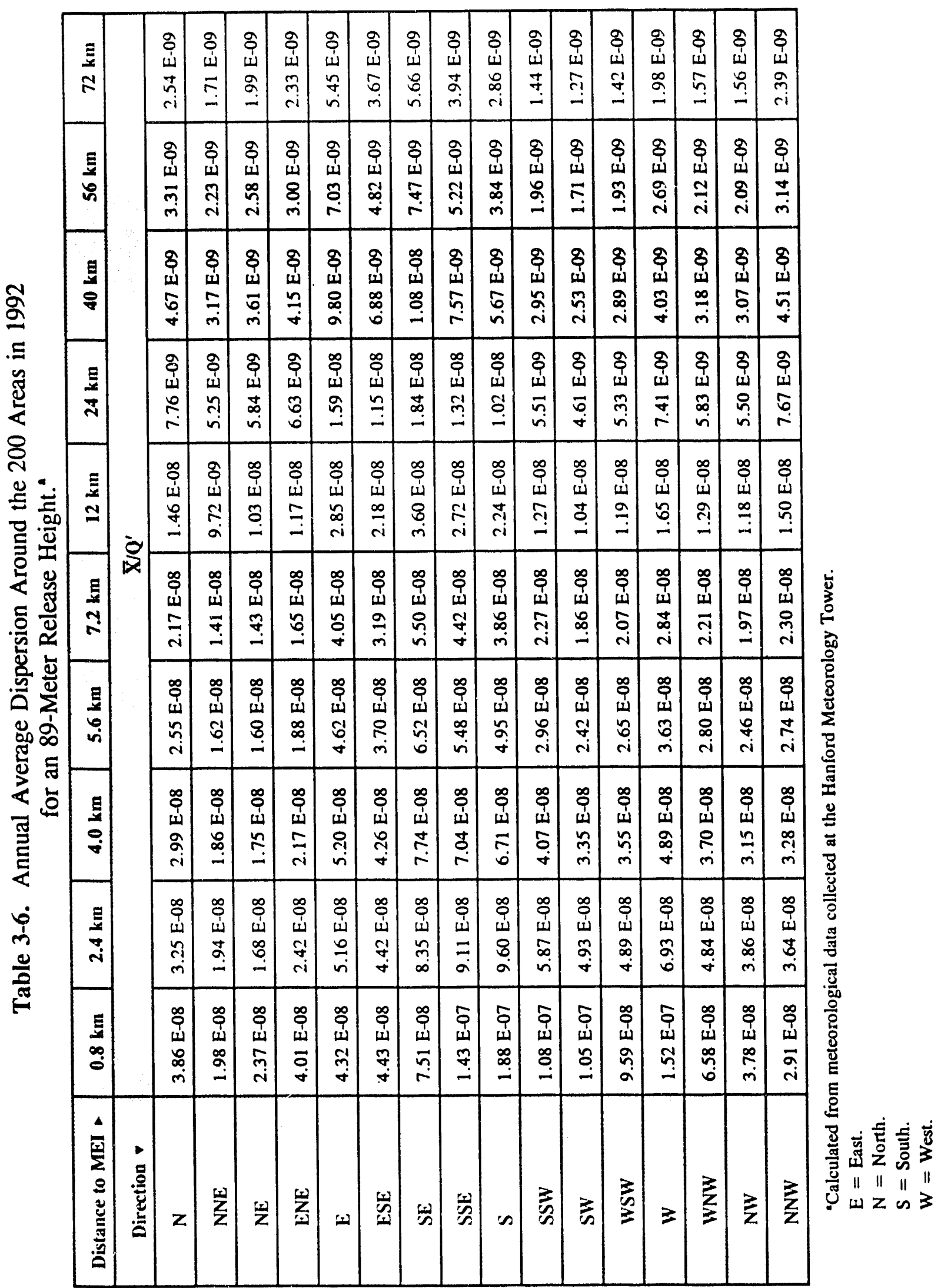




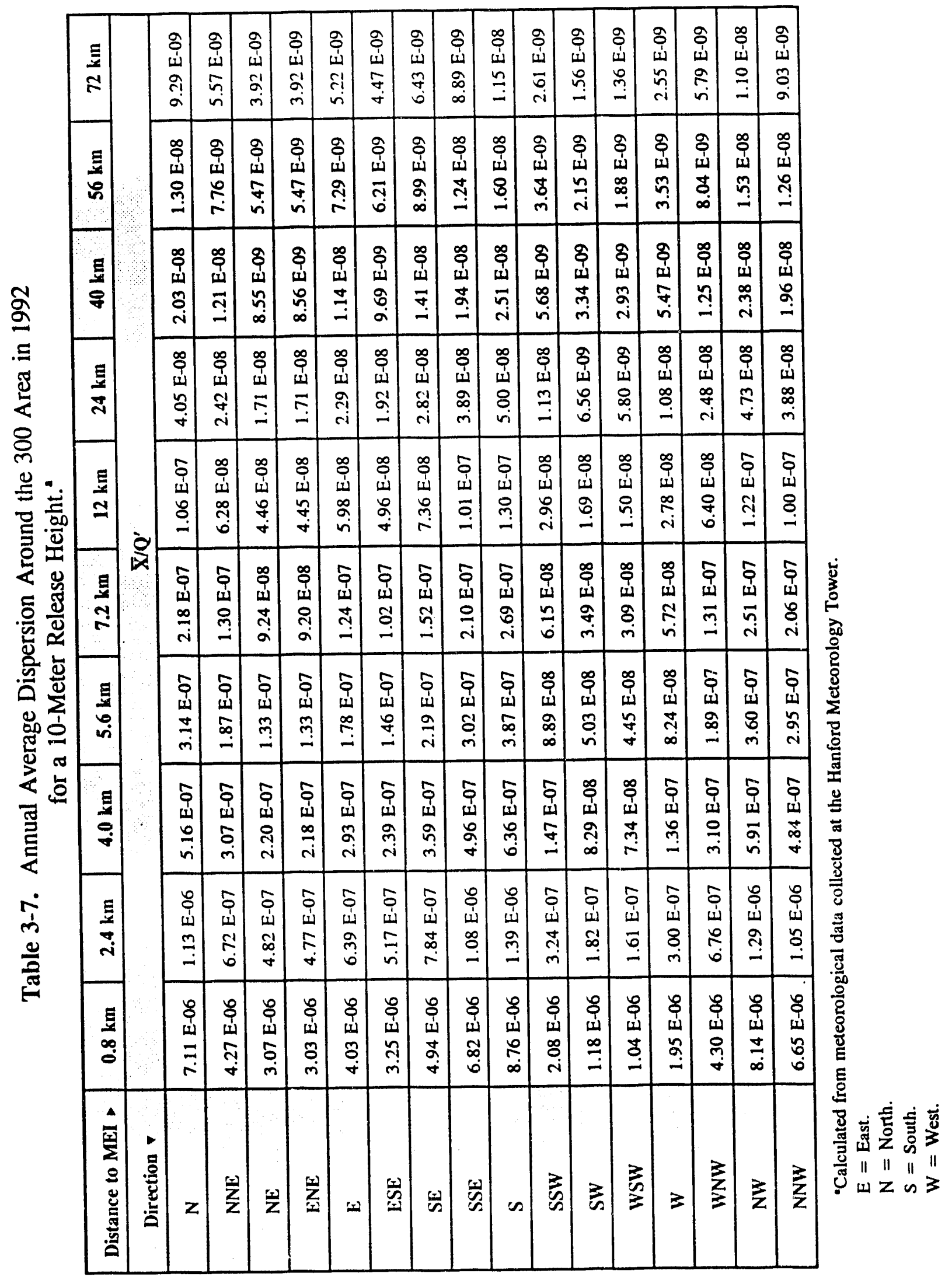




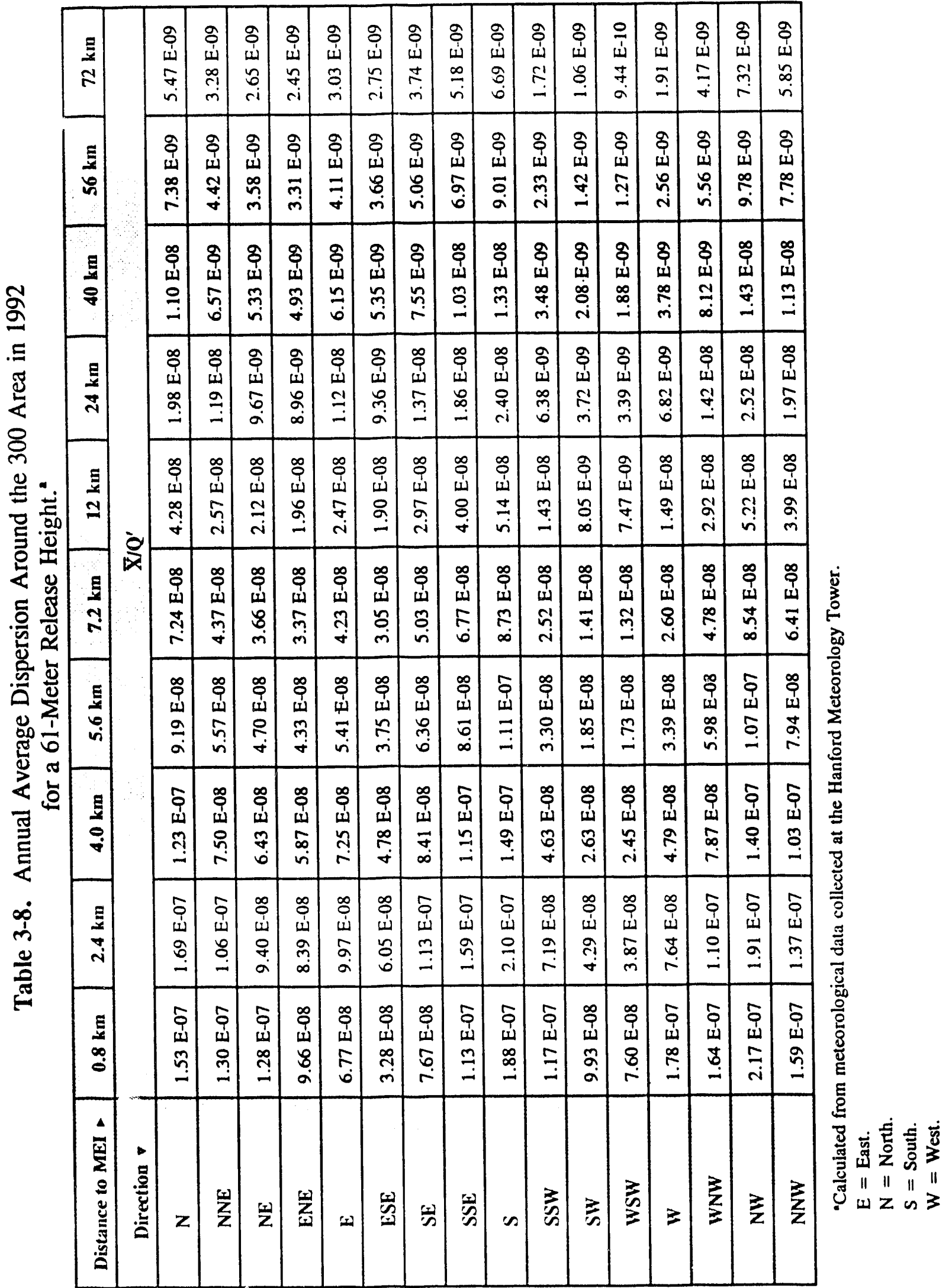




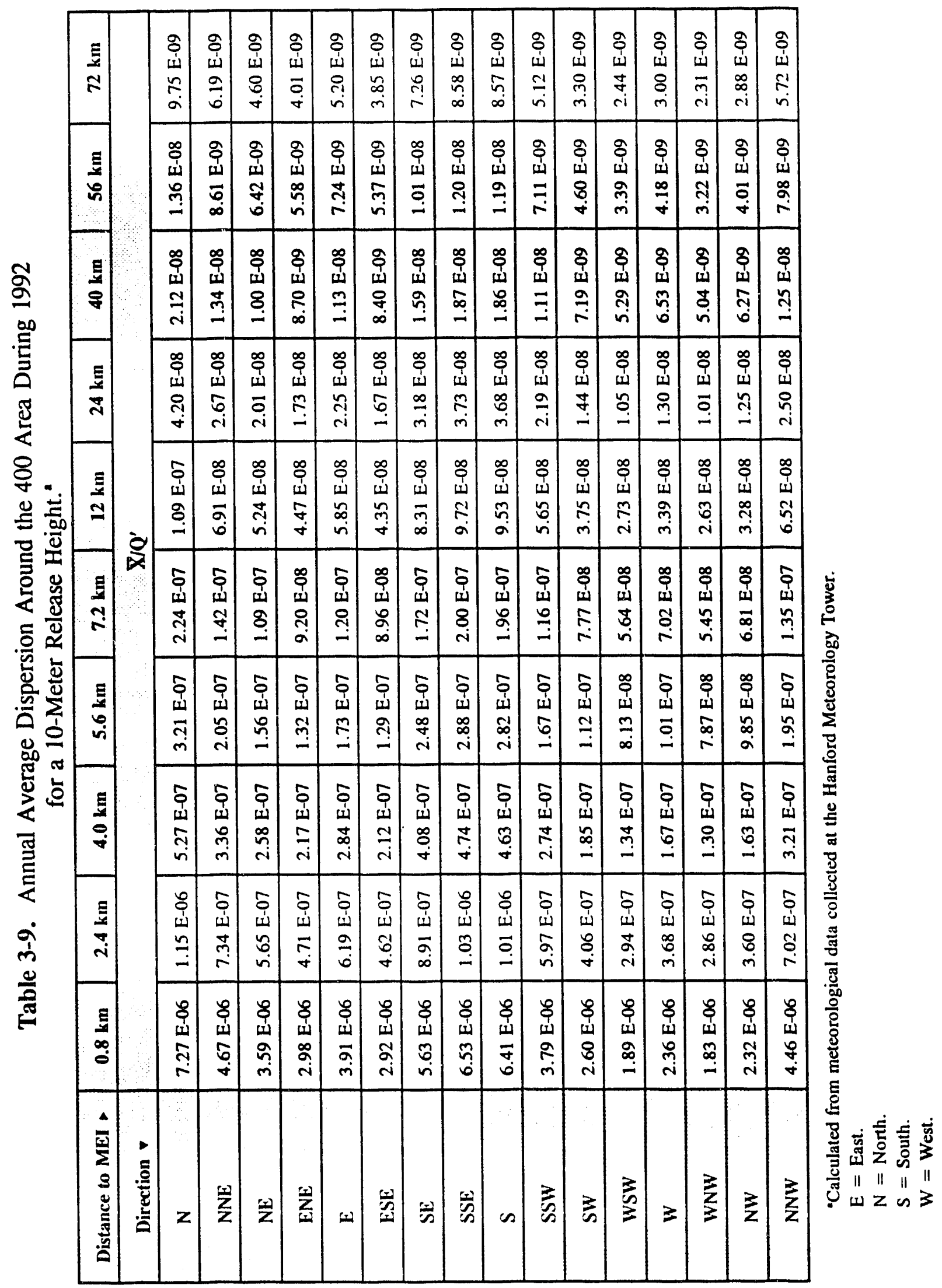




\subsection{CERTIFICATION}

This certification applies to Sections $1.0,2.0$, and 3.0 only.

"I certify under penalty of law that I have personally examined and am familiar with the information submitted herein and, based on my inquiry of those individuals immediately responsible for obtaining the information, I believe that the submitted information is true, accurate, and complete. I am aware that there are significant penalties for submitting false information, including the possibility of fine and imprisonment. See, 18 U.S.C. 1001."

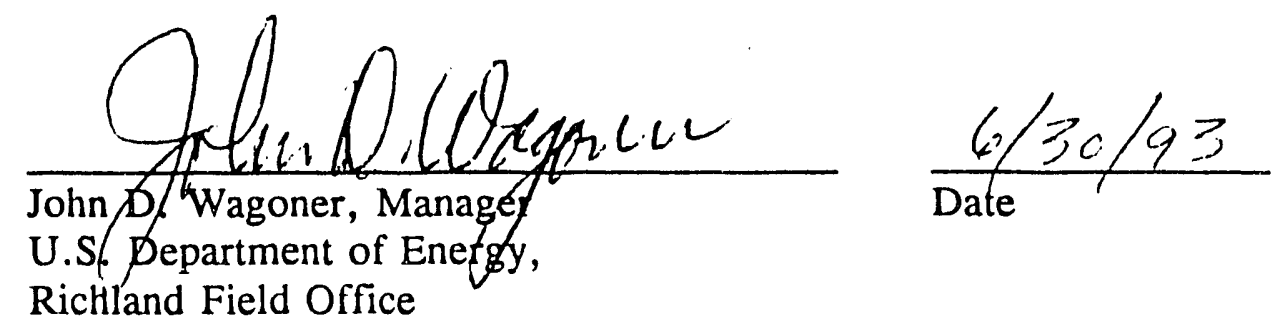


DOE/RL-93-36

This page intentionally left blank. 


\subsection{DIFFUSE-SOURCE EMISSIONS}

For inclusion in this report, DOE Headquarters has requested estimated doses to the public related to radioactive emissions from both point and diffuse sources. Previously, calculating public doses had been confined to emissions from point sources such as stacks. Data on diffuse emissions were contained appropriately in environmental monitoring results, which generally were too low to use for meaningful dose estimates. Instead, environmental monitoring data were reported simply as the activity of radionuclides detected rather than converting the activity to doses. This can be demonstrated by comparing environmental monitoring data with data on stack emissions in Hanford Site annual reports covering the last 35 years.

In the past, when the Hanford Site was operating at or near full capacity, point-source emissions were easily detected. Now, however, emissions from point sources have diminished in most instances to background levels. Methodologies for calculating doses from point sources are well established, based primarily on analyzing samples and measuring flow of stack emissions. Similar methodologies have not been developed for diffuse sources because of complicating factors such as: (1) difficulty in accurately quantifying air flow from the source, (2) more complex influences from meteorological conditions, and (3) exceedingly low detection levels. Currently, all nuclear material production facilities at the Hanford Site are shutdown or in standby mode. As an outcome, the environmental contribution from diffuse source emissions has become potentially a significant portion of the much lower total radioactivity attributable to activities at the Hanford Site.

For this report, doses have been calculated for emissions from both point and diffuse sources, even though the methods used for calculating diffuse emissions are still being refined. Dose calculations for each type of emission are provided separately.

As methods for estimating the dose from diffuse emissions are better refined, more precise amounts representing radionuclides occurring naturally and from nuclear-testing fallout (see Figure 4-1) may be subtracted from ambient air concentrations. Undoubtedly this would lower dose estimates.

\subsection{DIFFUSE EMISSIONS FROM UNMONITORED SOURCES}

Some air emissions from Hanford Site facilities with no radioactive source terms, such as office areas, are not monitored routinely. Similar types of unmonitored emission sources consist of support buildings, confinement zones, storage rooms, shops, and similar structures containing no radioactive materials available for release. Emissions forcibly discharged by exhaust fans are monitored if radioactive material can be potentially released.

Passive breather vents on various tanks, vaults, and other structures are potential conduits of radioactive emissions. But because airborne radionuclides inside vented 
Figure 4-1. Monthly Average Gross Beta Radioactivity in Airborne

Particulate Samples, 1981 through 1991 (Woodruff et al. 1992).

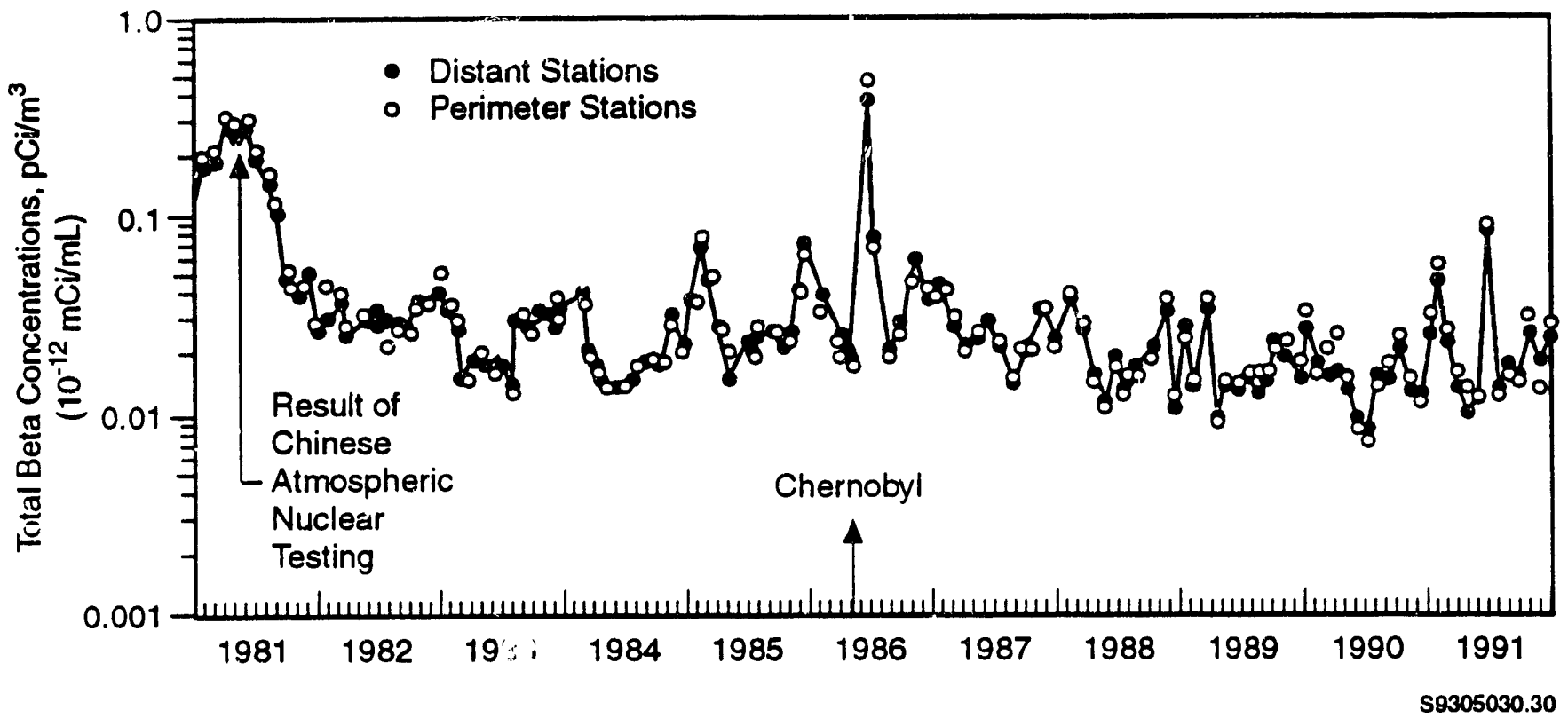

structures can be released only by passively exchanging with outside ambient air, emissions of this sort have been considerf d diffuse in nature because they lack a measurable flow. These potential emission sources are not monitored as a point-source stack is, but by using a comprehensive network of ambient air samplers. There are numerous types of these sources in and around the facilities described in Section 4.3. Dose and release estimates from unmonitored sources of radionuclide air emissions are included in Section 4.2 with the estimates related to diffuse sources.

Record sampling systems are installed on all stacks and vents with exhaust fans that forcibly cischarge potentially radioactive air. In almost all cases these stacks and vents (both large and small) are continuously sampled and the samples analyzed weekly. For the large majority of these discharge points, periodic confirmatory sampling would satisfy both EPA and DOE requirements. Nevertheless, these points are monitored much more stringently than if they were only periodically sampled, being sampled continuously and analyzed weekly for gross alpha and beta activity. The weekly measurements are averaged for the entire year, and $a: y$ positive result greater than zero, no matter how small, is included in the annual radionuclide air emissions report and modeling of offsite dose.

Just as Hanford Site operations have decreased in recent years, so have radionculide air emissions. The majority of the stacks and vents now exhaust air at or near background levels of radioactivity. 


\subsection{ESTIMATED RADIONUCLIDE AIR EMISSION RELEASES AND RESULTING DOSE TO ANY MEMBER OF THE PUBLIC FROM DIFFUSE AND UNMONITORED SOURCES}

The potential dose to an offsite individual and the potential releases from diffuse and unmonitored sources were estimated using ambient air monitoring data from locations around the perimeter of the Hanford Site. The Hanford Site air monitoring network consists of 50 sampling stations as follows: 23 are on the Site, 13 at the Site perimeter, 11 in nearby communities, and 3 in distant communities (Woodruff and Hanf 1992). The data used for this analysis were taken during 1991 and 1992 at eight of the perimeter sampling stations located in generally downwind directions (northeast to south) from the Site (Bisping and Wcodruff 1992).

\subsubsection{Methods for Assessing Diffuse-Source Emissions}

For a number of reasons, PNL has elected to use environmental monitoring data collected at the Site perimeter to estimate the dose from diffuse and unmonitored sources in preference to other possible methods. Despite the uncertainties inherent in low-level environmental sampling, measurements that best reflect dose at the receptor location are preferred over equally uncertain release estimates based on onsite monitoring data, or use of resuspension and dispersion models with questionable applicability to the Site. Information about the extent and levels of soil contamination on the Site is currently insufficient for resuspension and dispersion models for the dose estimates to be used with any confidence in their validity. Use of onsite monitoring data to estimate emissions from diffuse sources is also subject to substantial uncertainty because of a lack of accurate information about the potential source locations. An error in calculating release estimates based on inaccurate source location could potentially have a larger impact on the calculated offsite dose than the current uncertainties in the offsite air concentration measurement.

The ambient air sampling results consisted of annual average air concentrations for radionuclides that may be released from Site operations and diffuse sources, including tritium, iodine $\left({ }^{129} \mathrm{I},{ }^{131} \mathrm{I}\right)$, and particles $\left({ }^{90} \mathrm{Sr},{ }^{60} \mathrm{Co},{ }^{65} \mathrm{Zn},{ }^{95} \mathrm{Zr} /{ }^{95} \mathrm{Nb},{ }^{106} \mathrm{Ru} /{ }^{106} \mathrm{Rh},{ }^{125} \mathrm{Sb},{ }^{134} \mathrm{Cs}\right.$, ${ }^{137} \mathrm{Cs} /{ }^{137 \mathrm{~m}} \mathrm{Ba},{ }^{144} \mathrm{Ce} /{ }^{144} \mathrm{Pr},{ }^{154} \mathrm{Eu},{ }^{155} \mathrm{Eu}$, uranium isotopes, ${ }^{238} \mathrm{Pu}$, and ${ }^{239.240} \mathrm{Pu}$ ). The naturally occurring radionuclides ${ }^{7} \mathrm{Be}$ and ${ }^{40} \mathrm{~K}$ were also included in the analyses for particulate samples. Tritium, iodine, and uranium were only sampled at selected locations, and particulate samples for pairs of perimeter stations in the same general vicinity were pooled for radioanalysis to obtain more accurate counting statistics.

The contribution of diffuse and unmonitored sources to ambient air concentrations at each sample location was estimated from air monitoring data taken at that station, corrected for the contributions from stack emissions reported during 1991 and for regional background levels resulting from resuspension of naturally occurring radionuclides and fallout from weapons testing. When particulate samples from two stations were composited for analysis, the reported air concentration for the sample was assigned to the location of the station that would be expected to have a lower air concentration from emissions at onsite sources. This 
procedure eliminated the possibility that compositing of results from different stations would obscure a relatively high reading at one location, resulting in a more conservative estimate for the diffuse-source releases.

Radionuclide air concentrations resulting from 1991 and 1992 stack emissions were calculated for each sample location using the CAP-88 code package. The combined contributions to airborne radionuclide levels from all Hanford Site operating areas were subtracted from the ambient monitoring results for the corresponding years. The reference regional background level of each radionuclide was assumed to be equal to the average air concentration measured at distant community sampling stations in Yakima, Sunnyside, and Moses Lake, Washington. The average background concentration at these stations was also subtracted from the ambient monitoring results at perimeter stations to estimate the potential contribution from diffuse-source emissions.

\subsubsection{Results of Diffuse-Source Evaluation}

During 1991, the annual average ambient air concentrations of ${ }^{3} \mathrm{H},{ }^{90} \mathrm{Sr},{ }^{95} \mathrm{Zr},{ }^{106} \mathrm{Ru}$, ${ }^{137} \mathrm{Cs},{ }^{154} \mathrm{Eu}$, and ${ }^{238} \mathrm{Pu}$ were found to be numerically greater than the combined contributions for stack releases and background. Concentrations of other radionuclides may have been greater than stack releases and background at individual monitoring stations; however, the corrected average for these nuclides at all sampling stations was less than zero. A separate statistical comparison of the airborne radionuclide concentrations at perimeter sampling stations with those in distant communities indicated that only ${ }^{129} \mathrm{I}$ from stack emissions in the 200 Areas was significantly greater than regional background levels at the $5 \%$ confidence level during 1991 (Woodruff and Hanf 1992). Radionuclides that were elevated above expected levels from background and stack releases during 1992 included ${ }^{3} \mathrm{H},{ }^{90} \mathrm{Sr},{ }^{137} \mathrm{Cs}$, ${ }^{144} \mathrm{Ce}$, and total uranium.

Releases from diffuse and unmonitored sources were estimated from the corrected air concentrations by performing a back-calculation using CAP-88. The code was used to obtain the predicted doses and air concentrations at each monitoring location for a unit $(1 \mathrm{Ci} / \mathrm{yr})$ release of each sampled radionuclide at ground level from the 200 Areas. These results for diffuse emissions were then normalized to calculate a dose factor per unit air concentration (see Table 4-1), and a release quantity per unit air concentration at each downwind location.

The normalized ground-level releases per unit air concentration from CAP-88 calculations and the portion of the air concentrations measured at each sampling location that was attributable to diffuse and unmonitored sources were used to estimate the potential diffuse source emissions for each radionuclide. The 200 Areas were assumed to be the location of diffuse sources for all radionuclides except uranium, which may have been resuspended from contaminated soil in the 300 Area. Because it was not possible to determine the relative contributions from these potential sources, uranium releases were modeled as if all diffuse emissions originated at the 200 Areas, which results in a higher release estimate. The diffuse-source releases reported in Table 4-1 represent the mean of the release estimates for each of the selected downwind perimeter locations at which a particular 
Table 4.1. Estimated 1991 Hanford Site Diffuse Emissions and Resulting Dose (Sheet 1 of 2 )

\begin{tabular}{|c|c|c|c|}
\hline \multicolumn{3}{|c|}{$\begin{array}{l}\text { Location } \\
\text { [distance }(\mathrm{km}) \text { and direction to } 200 \text { Areas] }\end{array}$} & \multirow{2}{*}{$\begin{array}{l}\begin{array}{c}\text { Ringold Farm } \\
(26 \text { km ESE) }\end{array} \\
\text { Estimated Dose } \\
\text { at Location } \\
\text { (mrem/yr) }\end{array}$} \\
\hline Radionuclide $\mathbf{r}$ & $\begin{array}{l}\text { Dose Factor } \\
(\mathrm{mrem} / \mathrm{yr} \text { per } \\
\left.\text { pCi/m } \mathbf{m}^{3}\right)^{\mathbf{a}}\end{array}$ & $\begin{array}{l}\text { Diffuse-Source } \\
\text { Estimated Release } \\
\text { from } 200 \text { Areas } \\
\text { (Ci/yr) }\end{array}$ & \\
\hline${ }^{3} \mathrm{H}$ & $7.7 \mathrm{E}-03$ & $2.1 \mathrm{E}+02$ & $2.9 \mathrm{E}-03$ \\
\hline${ }^{6} \mathrm{Co}$ & $1.1 \mathrm{E}+01$ & $0.0 \mathrm{E}+00$ & 0.0 \\
\hline${ }^{65} \mathrm{Zn}$ & $3.6 \mathrm{E}+00$ & $0.0 \mathrm{E}+00$ & 0.0 \\
\hline${ }^{90} \mathrm{Sr}$ & $1.7 \mathrm{E}+01$ & $6.0 \mathrm{E}-01$ & $1.4 \mathrm{E}-02$ \\
\hline${ }^{95} \mathrm{Zr}$ & $1.0 \mathrm{E}+00$ & $2.6 \mathrm{E}-01$ & $3.5 \mathrm{E}-04$ \\
\hline${ }^{106} \mathrm{Ru} / \mathrm{Rh}$ & $7.6 \mathrm{E}+00$ & $1.3 \mathrm{E}+00$ & $1.3 \mathrm{E}-02$ \\
\hline${ }^{125} \mathrm{Sb}$ & $1.6 \mathrm{E}+00$ & $0.0 \mathrm{E}+00$ & 0.0 \\
\hline${ }^{129} I$ & $3.0 \mathrm{E}+02$ & $0.0 \mathrm{E}+00$ & 0.0 \\
\hline${ }^{131} I$ & $1.7 E+01$ & $0.0 \mathrm{E}+00$ & 0.0 \\
\hline${ }^{134} \mathrm{Cs}$ & $1.2 \mathrm{E}+01$ & $0.0 \mathrm{E}+00$ & 0.0 \\
\hline${ }^{137} \mathrm{Cs} / \mathrm{Ba}$ & $7.4 \mathrm{E}+00$ & $2.2 \mathrm{E}-02$ & $2.1 \mathrm{E}-04$ \\
\hline${ }^{144} \mathrm{Ce}$ & $5.4 \mathrm{E}+00$ & $0.0 \mathrm{E}+00$ & 0.0 \\
\hline${ }^{154} \mathrm{Eu}$ & $7.2 \mathrm{E}+00$ & $5.1 \mathrm{E}-01$ & $4.7 \mathrm{E}-03$ \\
\hline${ }^{155} \mathrm{Eu}$ & $7.2 \mathrm{E}-01$ & $0.0 \mathrm{E}+00$ & 0.0 \\
\hline U Total & $1.3 \mathrm{E}+03$ & $0.0 \mathrm{E}+00$ & 0.0 \\
\hline${ }^{238} \mathrm{Pu}$ & $3.2 \mathrm{E}+03$ & $1.5 E-05$ & $6.0 \mathrm{E}-05$ \\
\hline${ }^{239,240} \mathrm{Pu}$ & $3.4 E+03$ & $0.0 \mathrm{E}+00$ & 0.0 \\
\hline & Total & & $3.5 \mathrm{E}-02$ \\
\hline
\end{tabular}

'Dose factor includes ingestion, inhalation, air submersion, and external exposure to radionuclides on ground

${ }^{b}$ Emissions from diffuse sources assumed to originate in the Hanford Site 200 Areas, except for uranium. The 300 Area also has potential sources for resuspension of uranium from soil, in addition to naturally occurring uranium isotopes found throughout the area. Because it was not possible to determine the source of uranium isotopes detected at offsite sample stations, the releases were modeled as if the total inventory were from the 200 Areas.

'Dose is based on air monitoring results for sample stations downwind, generally east and south, of the Hanford Site. The measured air concentrations for these locations are corrected for estimated contributions from stack releases and regional background levels of monitored radionuclides. Doses that are reported as being $<0$ result from air monitoring results that are below the detection limit or for which the monitoring results are lower than the contributions from stack releases and background. 
Table 4.1. Estimated 1992 Hanford Site Diffuse Emissions and Resulting Dose (Sheet 2 of 2)

\begin{tabular}{|c|c|c|c|}
\hline \multicolumn{3}{|c|}{$\begin{array}{l}\text { Location - } \\
\text { [distance (km) and direction to } 200 \text { Areas] }\end{array}$} & \multirow{2}{*}{$\begin{array}{c}\text { Ringold Farm } \\
(26 \mathrm{~km} \text { ESE) } \\
\begin{array}{c}\text { Estimated Dose } \\
\text { at Location } \\
(\mathrm{mrem} / \mathrm{yr})^{\circ}\end{array}\end{array}$} \\
\hline Radionuclide $>$ & 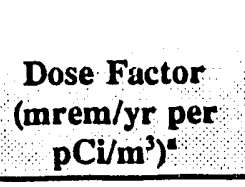 & $\begin{array}{l}\text { Diffuse-Source } \\
\text { Estimated Release } \\
\text { from } 200 \text { Areas } \\
(\mathrm{Ci} / \mathrm{yr})^{\mathrm{b}}\end{array}$ & \\
\hline${ }^{3} \mathrm{H}$ & $7.5 \mathrm{E}-03$ & $6.4 \mathrm{E}+03$ & $8.7 \mathrm{E}-02$ \\
\hline${ }^{60} \mathrm{Co}$ & $1.1 \mathrm{E}+01$ & 0.0 & 0.0 \\
\hline${ }^{65} \mathrm{Zn}$ & $3.6 \mathrm{E}+00$ & 0.0 & 0.0 \\
\hline${ }^{90} \mathrm{Sr}$ & $1.7 \mathrm{E}+01$ & $8.2 \mathrm{E}-04$ & $1.8 \mathrm{E}-05$ \\
\hline${ }^{95} \mathrm{Zr}$ & $1.0 \mathrm{E}+00$ & 0.0 & 0.0 \\
\hline${ }^{106} \mathrm{Ru} / \mathrm{Rh}$ & $7.5 \mathrm{E}+00$ & 0.0 & 0.0 \\
\hline${ }^{125} \mathrm{Sb}$ & $1.6 \mathrm{E}+00$ & 0.0 & 0.0 \\
\hline${ }^{129} \mathrm{I}$ & $3.0 \mathrm{E}+02$ & 0.0 & 0.0 \\
\hline${ }^{131} I$ & $1.7 \mathrm{E}+01$ & 0.0 & 0.0 \\
\hline${ }^{134} \mathrm{Cs}$ & $1.2 \mathrm{E}+01$ & 0.0 & 0.0 \\
\hline${ }^{137} \mathrm{Cs} / \mathrm{Ba}$ & $7.3 \mathrm{E}+00$ & $3.3 \mathrm{E}-03$ & $3.1 \mathrm{E}-0.5$ \\
\hline${ }^{144} \mathrm{Ce}$ & $5.3 \mathrm{E}+00$ & $1.6 \mathrm{E}-00$ & $1.1 \mathrm{E}-03$ \\
\hline${ }^{154} \mathrm{Eu}$ & $7.1 \mathrm{E}+00$ & 0.0 & 0.0 \\
\hline${ }^{155} \mathrm{Eu}$ & $7.2 \mathrm{E}-01$ & 0.0 & 0.0 \\
\hline U Total & $1.2 \mathrm{E}+03$ & $1.4 \mathrm{E}-03$ & $2.3 \mathrm{E}-03$ \\
\hline${ }^{238} \mathrm{Pu}$ & $3.1 E+03$ & 0.0 & 0.0 \\
\hline${ }^{239,240} \mathrm{Pu}$ & $3.4 E+03$ & 0.0 & 0.0 \\
\hline \multicolumn{3}{|c|}{ Total (all radionuclides) ${ }^{d}$} & $9.0 \mathrm{E}-02$ \\
\hline \multicolumn{3}{|c|}{ Total (all radionuclides except ${ }^{3} \mathrm{H}$ ) } & $3.4 \mathrm{E}-02$ \\
\hline
\end{tabular}

'Dose factor includes ingestion, inhalation, air submersion, and external c:sposure to radionuclides on ground

${ }^{b}$ Emissions from diffuse sources assumed to originate in the Hanford Site 200 Areas, except for uranium. The 300 Area also has potential sources for resuspension of uranium from soil, in addition to naturally occurring uranium isotopes found throughout the area. Because it was not possible to determine the source of uranium isotopes detected at offsite sample stations, the releases were modeled as if the total inventory were from the 200 Areas.

${ }^{c}$ Dose is based on air monitoring results for sample stations downwind, generally east and south, of the Hanford Site. The measured air concentrations for these locations are corrected for estimated contributions from stack releases and regional background levels of monitored radionuclides. Doses reported as being < 0 result from air monitoring results that are below the detection limit or for which the monitoring results are lower than the contributions from stack releases and background.

These estimates for ${ }^{3} \mathrm{H}$ are based in air monitoring results that may have been affected by contamination of air samples in the analytical laboratory during the first six months of 1992. 
radionuclide was sampled. In accordance with guidance from DOE-HQ, the average release estimates for diffuse sources were calculated using air concentrations that were both positive and negative with respect to the regional background and stack emission estimates. If the resulting mean release estimate for a particular radionuclide was less than zero, it was reported as zero in Table 4-1.

The estimated diffuse-source releases for the sampled radionuclides were also used to estimate the dose at each downwind perimeter sampling station and at the location of the Ringold farm selected for the MEI for stack emissions. When the corrected air estimates and resulting doses for a particular radionuclide were numerically less than zero, the dose estimates were set equal to zero before adding them to obtain the total dose at each location. The results in Table 4-1 indicate that the hypothetical dose at individual perimeter monitoring stations ranged from 0.011 to $0.064 \mathrm{mrem} / \mathrm{yr}$, and was $0.035 \mathrm{mrem} / \mathrm{yr}$ at the Ringold farm. This is somewhat greater than the estimated dose from stack emissions during 1991, which was 0.007 mrem at the Ringold receptor location. Based on these results, the combined dose from stack emissions and diffuse and unmonitored sources during 1991 was substantially below the $10 \mathrm{mrem} / \mathrm{yr}$ standard.

Air sampling data from 1992 yielded somewhat higher dose estimates for diffuse and unmonitored sources, in part because of suspected cross-contamination of tritium air samples in the analytical laboratory. Elevated tritium concentrations were found sporadically throughout the year, but were most common during the first five months. Samples from both perimeter and distant community stations were affected; however, not all were elevated to the same degree. When all tritium sample data are included, the estimated dose from diffuse and unmonitored sources at six individual perimeter monitoring stations ranged from 0.09 to $0.18 \mathrm{mrem} / \mathrm{yr}$. The dose to the site receptor at the Ringold farm was $0.09 \mathrm{mrem} / \mathrm{yr}$ which, as in 1991, was greater than the estimated dose at that location from stack emissions during 1992 (0.004 mrem). If the tritium data are excluded entirely, the dose at all locations from the remaining radionuclides amounts to less than $0.01 \mathrm{mrem} / \mathrm{yr}$. Based on these results, the combined dose from stack emissions and diffuse and unmonitored sources during 1992 was also well below the EPA standard.

\subsubsection{Estimate of Uncertainty in Dose from Diffuse Sources}

To obtain an estimate of the uncertainty in the dose estimates for perimeter locations, the reported air concentrations at the Fir Road sampling station and at the distant community stations (including the contributions from diffuse sources, regional background, and stack releases) were used to estimate the mean and $95 \%$ confidence intervals for each location. The Fir Road station was selected for this analysis because it is closest to the location of the Ringold farm receptor and has similar predicted air concentrations for transport from onsite sources. The calculation was performed using the GENII-S computer code (Leigh et al. 1992) to produce a stochastic analysis of the environmental radiation doses. The mean values of the measured air concentrations were entered as basic concentrations along with the maximum and minimum sample values to define a triangular distribution for each 
radionuclide. The code then used a Latin hypercube sampling routine to select random values for each radionuclide concentration in 100 trials to obtain the dose distribution for each location.

The estimated mean dose for the Fir Road station was $0.13 \mathrm{mrem} / \mathrm{yr}$ for artificially produced radionuclides sampled at that location, with a $95 \%$ confidence interval of 0.027 to $0.23 \mathrm{mrem} / \mathrm{yr}$. The corresponding result for the distant community monitoring stations was $0.14 \mathrm{mrem} / \mathrm{yr}$ with $95 \%$ confidence limits of 0.0 to $0.25 \mathrm{mrem} / \mathrm{yr}$, virtually indistinguishable from the results for the perimeter station. Because the measured dose at perimeter stations was almost entirely a result of background radioactive materials (naturally occurring radionuclides and fallout), the diffuse-source component was a relatively small fraction of the total. The $95 \%$ confidence limits for the dose from diffuse sources would therefore be a small fraction of those for the total dose at these locations.

A similar analysis for 1992 data used sample results from the Byers Landing station because it was the only perimeter location for which all radionuclides were reported. The predicted air concentrations at this station from onsite emissions are very similar to those at Fir Road and the Ringold Receptor. Using tritium data from June through December only (the samples least affected by the suspected contamination) and annual average concentrations for all other artificially produced radionuclides, the estimated mean dose at Byers Landing was $0.083 \mathrm{mrem} / \mathrm{y}$, with a $95 \%$ confidence interval of 0.029 to $0.13 \mathrm{mrem} / \mathrm{yr}$. At the distant community monitoring stations, the average dose was $1.2 \mathrm{mrem} / \mathrm{yr}$ and the $95 \%$ confidence interval was 0.23 to $2.5 \mathrm{mrem} / \mathrm{yr}$. In this case, the dose for the perimeter station was similar to those estimated for 1991 . However, the $199 \%$ values for the distant community stations were significantly higher than those for the site perimeter because of an apparently anomalous high tritium sample collected during July. As in 1991, the hypothetical diffuse source component was a relatively small fraction of the total dose from airborne radionuclides measured at any offsite location, and the $95 \%$ confidence limits for dose from diffuse sourcss would be substantially lower than those for the total.

\subsubsection{Discussion}

The release estimates for diffuse and unmonitored sources in Table 4-1 were obtained using the CAP-88 code package, which incorporates a continuous release Gaussian plume dispersion model. Releases from diffuse sources would be expected to occur primarily under conditions that are very different from the annual average assumptions used in the CAP-88 model. This is particularly true for resuspension of contaminated soil, which is a function of the wind speed, and to some extent for emissions from sources such as evaporation ponds. Because release rates from such sources are greatest under conditions that favor atmospheric dispersion, use of an annual average continuous-release model to back-calculate the release quantities may introduce a significant bias in these estimates. The dose estimates for sources of this type may also be affected by seasonal variation in the resuspension rates caused by the prevalence of strong winds during certain seasons of the year. If these episodes occur primarily during times when crop production is minimal, some of the exposure pathways incorporated into the CAP-88 code (direct deposition on human and animal food crops, for 
example) would not be applicable. The release and dose estimates reported for diffuse sources in this evaluation should therefore be viewed as approximations whose accuracy is limited by a number of factors inherent in the sampling and modeling process.

\subsection{DIFFUSE-EMISSION SOURCES}

The Hanford Site consists of $1,450 \mathrm{~km}^{2}\left(560 \mathrm{mi}^{2}\right)$ of semiarid shrub-steppe land, of which approximately $6 \%$ has been disturbed and actively used. This $6 \%$ of land [about $83 \mathrm{~km}^{2}\left(32 \mathrm{mi}^{2}\right)$, or 8,090 ha $(20,000$ acres $)$ ] is divided into large operational and support areas: the $100,200,300,400$, and 1100 Areas. The 600 Area designates the remaining land outside the operational and support areas. Almost all point and diffuse sources of radionuclide emissions are located in the five operational areas; two minor point sources are in the 600 Area. Point sources are measured directly or calculated from process knowledge. Diffuse sources are measured through a network of monitoring systems extending from the potential and actual sources to far offsite.

The Hanford Site was acquired in 1943 and dedicated to producing plutonium for national defense and managing the resulting production wastes. Restoring the Hanford Site environment is the new mission that has largely supplanted the previous operational objectives. The environmental restoration effort will entail activities such as decontaminating and decommissioning over 100 facilities and cleaning up and restoring about 1,500 waste sites. Until the restoration and cleanup work is completed, radioactive emissions may be released from hundreds of diffuse sources, in addition to known point-source stacks.

Besides measuring point source emissions and modeling those emissions to determine public doses, environmental surveillance is conducted. Environmental and food-chain pathways are monitored near facilities emitting radionuclides from either point sources or diffuse sources. The environmental pathways for all air emissions from the Hanford Site are monitored using a stratified sampling approach. Samples are collected and radiation is measured according to three surveillance zones. These zones extend from main onsite operating areas to offsite regions (Woodruff et al. 1992).

The first zone begins near the operating facilities and ends at the Hanford Site perimeter. Diffuse emissions generally will be most concentrated and easier to detect in this zone before diluting further as they drift offsite.

The second surveillance zone is a series of sampling stations that surround the Hanford Site near its perimeter. Because a person could live as close to the Hanford Site as some of these stations, their data represent the maximum exposures for a member of the public. Therefore, ambient air sampling data from the perimeter locations most closely reflect the actual impacts of radionuclide air emissions from point sources and diffuse sources on the Hanford Site. 
The third surveillance zone encompasses nearby and distant communities within an $80-\mathrm{km}$ radius of the center of the Hanford Site but beyond its boundaries. Surveillance is conducted in communities to provide measurements at those locations where the most people are potentially exposed. This surveillance ensures that radionuclide levels are well below standards established to protect the public health. Finally, background concentrations are measured at distant locations and compared with onsite, perimeter, and community locations to indicate the effects of Hanford Site activities. Background locations aite essentially unaffected by Hanford Site emissions but contain similar levels of radioactivity originating naturally and from nuclear-testing fallout.

The goal of environmental surveillance at the Hanford Site is to verify compliance with DOE and EPA radiological dose standards for public protection. This goal is accomplished by measuring radionuclides and consequent exposure in the onsite and offsite environment. The environmental surveillance criteria are derived from: (1) the collected environmental surveillance data on radionuclides and doses, (2) applicable regulations other than DOE Orders, (3) DOE Order 5400.1 (DOE 1988), and (4) the DOE Environmental Regulatory Guide for Radiological Effluent Monitoring and Environmental Surveillance (DOE 1991). The surveillance program (Woodruff et al. 1991) was established on these criteria and the pathway analyses that provide information on radionuclides and media contributing to human dose. Experience from Hanford Site environmental surveillance activities and studies conducted over the past 45 years has built an invaluable technical background of information for planning and data interpretation.

\subsubsection{Sources of Diffuse Emissions}

The presently identified actual or potential sources of diffuse radionuclide emissions to the environment at the Hanford Site are described in this section. Among the sources that could contribute diffuse radionuclide emissions are several kinds of waste handling and disposal facilities used at the Hanford Site such as cribs, ponds, ditches, trenches, retention basins, valve pits, French drains, reverse wells, tanks, and burial grounds. Operating facilities or facilities on standby or that are inactive also could contribute diffus ' emissions. Tables 4-2 and 4-3 show the types of waste and the types of diffuse sources, respectively. Each site or facility usually has one or more unique features or characteristics that may contribute to the release of diffuse emissions. Features may include passive vents, risers, equipment and personnel access doors, and exhausters. Characteristics may include an undetected leak, unburied waste, or an absence of intrusion barriers. Rates of diffuse emissions may be influenced by a variety of environmental conditions, such as: (1) changing atmospheric pressures, (2) wind speed, (3) erosion, (4) evaporation, (5) percolation, (6) biotic intrusion, or (7) wind-caused resuspension.

The general types of sites and facilities and their potential primary sources of diffuse emissions are briefly described in sections 4.3.1.1 through 4.3.1.12 that follow. 
Table 4-2. Types of Waste and Number of Waste Sites at the Hanford Site.

\begin{tabular}{|c|c|}
\hline Types of Waste & Number of Waste Sites \\
\hline Nonhazardous & 184 \\
\hline Hazardous & 142 \\
\hline Radioactive & 146 \\
\hline Mixed & 949 \\
\hline Total & $\mathbf{1 , 4 2 1}$ \\
\hline
\end{tabular}

Table 4-3. Types of Diffuse Sources.

\begin{tabular}{|l|c|c|}
\hline Types of Diffuse Sources & Active & Inactive \\
\hline Tanks & 28 & 149 \\
\hline Cribs & 16 & 115 \\
\hline $\begin{array}{l}\text { Trenches, ponds, ditches, } \\
\text { retention basins }\end{array}$ & 21 & 120 \\
\hline Reverse wells & 0 & 11 \\
\hline French drains & 21 & 47 \\
\hline Unplanned spill sites & 0 & 229 \\
\hline Burial grounds & 9 & 68 \\
\hline Total & 95 & $\mathbf{7 3 9}$ \\
\hline
\end{tabular}


4.3.1.1 Crib. Low-level liquid waste is discharged to the ground by structures called cribs. These are generally subsurface systems, similar to sanitary drain fields, that allow the liquid component of the waste to percolate into the soil. The natural properties of the soil are used to remove radioactive material from the effluent water through filtration, ion-exchange, and precipitation reactions.

Many cribs are vented to the atmosphere through engineered structures called vents and pipe risers. These engineered structures promote downward flow of liquids disposed of in the cribs but also provide pathways to the surface and atmosphere. Secondary causes of diffuse emissions include erosion, uptake and intrusion by biota, followed by wind-caused resuspension.

4.3.1.2 Pond. Ponds are used to manage the large quantities of water (i.e., cooling water and chemical-sewer wastewater) associated with chemical processing operations. Normally, these liquid effluents are uncontaminated. The ponds allow percolation of the liquid effluent into the soil column. Diffuse emissions from ponds are caused primarily by wind resuspension.

4.3.1.3 Ditch. A ditch is an open, unlined excavation used for disposing of liquid effluents or transporting liquid effluents to ponds for disposal. Diffuse emissions from ditches are primarily caused by wind resuspension.

4.3.1.4 Trench. Early disposal practices included disposing of liquid effluents into unlined trenches and filling them with soil over time. These were mostly replaced by cribs such as the BC-cribs. Diffuse emissions from trenches are primarily caused by erosion, uptake and intrusion by biota, followed by wind resuspension.

4.3.1.5 Retention Basin. Similar to trenches, retention basins generally were lined with concrete and used to hold liquids before routing them to ditches or ponds. Diffuse emissions from retention basins are caused primarily by wind resuspension.

4.3.1.6 Diversion Box. A diversion box is usually an underground, concrete structure formed around a junction of transfer lines carrying liquid effluent. When diversion boxes are accessed for operations or maintenance, radioactively contaminated material may be released in the form of diffuse emissions.

4.3.1.7 Valve Pit. A valve pit is similar in structure to diversion box, but contains piping valves. When valve pits are accessed for maintenance or operations, radioactively contaminated material may be released in the form of diffuse emissions.

4.3.1.8 French Drain and Reverse Well. A French drain is a rock-filled encasement inserted in the ground. A reverse well is an ordinary well used for mixing liquid waste with groundwater. These subsurface systems manage potentially contaminated liquid waste by promoting percolation into the soil. The natural filtration properties of the soil remove radioactive material from effluent water. Diffuse emissions from French drains and reverse 
wells may occur through erosion, uptake and intrusion by biota, followed by wind-caused resuspension.

4.3.1.9 Tank. A tank generally is a large reinforced metal structure that receives liquid effluent for storage. Examples are the DSTs and SSTs in the 200 Areas, which typically are arrayed in clusters called tank farms. Pathways for diffuse emissions from tanks include vents and exhausters open to the atmosphere and deposition and resuspension.

4.3.1.10 Burial Ground. Burial grounds are shallow trenches in which contaminated solid waste is buried. This waste is generated by various activities on the Hanford Site. Waste packaging procedures and burial practices used depend on the type of waste. Diffuse emissions occur at burial grounds through direct release to the atmosphere before the waste is buried, followed by erosion, uptake and intrusion by biota, and wind-caused resuspension.

\subsubsection{Decontamination and Decommissioning Activities. Westinghouse Hanford} activities also involve decontaminating and decommissioning retired facilities, equipment, and waste disposal sites. These activities are aimed at preventing the release or spread of contamination and reducing the number of acres of surface contamination. In some cases, contaminated material may be exposed to the atmosphere, bui steps are taken to isolate and reduce spreading of contamination as it is identified. Diffuse emissions are caused primarily by exposing contaminated surfaces such as the inside of contaminated structures. Planning and using procedures usually minimize these exposures, however. Increased monitoring around demolition activities can detect heightened levels of contamination on surfaces and in the atmosphere. To date, no significant increases at demolition sites have been observed.

\subsubsection{Radioactively Contaminated Surface Areas. All of the following radioactive} contaminated areas are routinely surveyed: burial grounds, cribs, trenches, retention basins, and unplanned release sites. The surveys are performed at least annually, but more frequently when needed. The number of surface-contaminated acres varies. The number is not fixed because there is a continuing effort to clean, stabilize, or remediate known contaminated areas while new areas of contamination are continuing to be identified. Newly identified contamination may be because of preexisting contamination that has migrated, by way of wind or biological intrusion, to previously uncontaminated areas or because the radiological criteria have become more stringent. Eighty percent of all areas of surface contamination are estimated to have dose rates less than $1 \mathrm{mrem} / \mathrm{h}$.

Contaminated areas are posted as "Surface Contamination Area," "Radiologically Controlled Area," or "Underground Radioactive Material." Radiologically controlled areas are large areas with a potential for speck contamination. Surface contamination areas have more widespread contamination. "Underground Radioactive Material" signs mark cribs, burial grounds, covered trenches, and ponds, but not underground plumes that extend away from these sites. If an area has surface and underground contamination, such as a surface contaminated crib, it will have both postings. The general location, by area, and the approximate area of surface contamination and volume of underground contamination are 
shown in Table 4-4. Diffuse emissions from areas of surface contamination are caused primarily by erosion, uptake and intrusion by biota, followed by wind-caused resuspension.

Table 4-4. Hanford Site Surface and Underground Contamination.

\begin{tabular}{|c|c|c|}
\hline Hanford Site Area & $\begin{array}{c}\text { Surface Contamination* } \\
\text { (acres [ha]) }\end{array}$ & $\begin{array}{c}\text { Underground Radioactive } \\
\text { Material }^{\mathbf{b}} \\
\text { (acres [ha]) }\end{array}$ \\
\hline 100 Areas & $160(65)$ & $460(186)$ \\
\hline 200 Areas & $329(133)$ & $366(148)$ \\
\hline 600 Area & $2,620(1,060)$ & $260(105)$ \\
\hline 300 Area (north) & $45(18)$ & $30(12)$ \\
\hline Total & $\mathbf{3 , 1 5 4 ( 1 , 2 7 0 )}$ & $\mathbf{1 , 1 1 6 ( 4 5 1 )}$ \\
\hline
\end{tabular}

"Includes areas posted as "Surface Contamination Areas" or as

"Radiological Controlled Areas" and areas that have both underground and surface contamination.

bonly includes areas with underground contamination, exclusive of groundwater plumes.

The following sections (4.3.1.13 through 4.3.1.32) briefly describe the sites and facilities considered as potential or known sources of diffuse emissions and the radionuclides primarily associated with them.

4.3.1.13 100-K Area Basins. Two identical reactors are located in the 100-K Area. One reactor is in the 100-KE Area while the other is in the 100-KW Area. The reactors and their support facilities were constructed between 1952 and 1954, beginning service in 1955. The $100-\mathrm{KW}$ Area reactor ceased operating in 1970 and the 100-KE reactor in 1971.

The 100-KE and $-\mathrm{KW}$ reactor systems underwent $\mathrm{D} \& \mathrm{D}$ (decontamination and decommissioning) after shutdown. Most of the fuel was sent to 200 East Area for processing. After the initial D\&D was completed, the fuel storage basins within the $105-\mathrm{KE}$ and $105-\mathrm{KW}$ buildings were modified to store $\mathrm{N}$ Reactor irradiated fuel. Storing this fuel began in 1975 at $105-\mathrm{KE}$ and in 1981 at $105-\mathrm{KW}$; fuel is still stored in both basins. Shipments of fuel to the basins from 100-N Area ceased in 1989.

The primary radionuclides that may be included in diffuse emissions from the $100-\mathrm{K}$ Basins include ${ }^{60} \mathrm{Co},{ }^{90} \mathrm{Sr},{ }^{137} \mathrm{Cs}$, and ${ }^{239,240} \mathrm{Pu}$. The potential primary release points of diffuse emission include personnel and equipment access doors, which are normally closed. 
4.3.1.14 $\mathrm{N}$ Reactor. The $\mathrm{N}$ Reactor was designed to produce special nuclear materials (SNM) and byproduct steam for electrical power generation. It began operations in 1963, and ceased operating in January 1987. The 100-N Area reactor complex consists of 126 buildings for reactor operations, maintenance, support facilities, and offices. Some of the more important reactor operations facilities with radioactive materials are identified below.

The 105-N Reactor Building contains the reactor, the reactor control room, the fuel loading area, and the spent fuel storage basin. The $\mathbf{N}$ Reactor is a graphite-moderated, lightwater-cooled, horizontal-pressure-tube nuclear reactor designed to produce SNM using slightly enriched uranium. Radioactively contaminated facilities associated with the N Reactor are:

- The 1301-N and 1325-N Liquid Waste Disposal Facilities consists of a crib and trench.

- The 109-N Heat-Exchanger Building transferred the heat from the primary loop to the secondary loop through steam generators.

- The 117-N Filter Building houses charcoal and HEPA filters.

- The 107-N Basin Recirculating Building removes radionuclides from the $\mathrm{N}$ Basin Fuel Storage Facility water by filtering and demineralizing it.

The primary radionuclides that may be included in diffuse emissions from the $100-\mathrm{N}$ facilities include ${ }^{60} \mathrm{Co},{ }^{90} \mathrm{Sr},{ }^{137} \mathrm{Cs}$, and ${ }^{239.240} \mathrm{Pu}$. The potential primary diffuse emission release points include railroad rollup doors, personnel and other equipment access doors (all of which are normally closed), and inactive exhaust vents and risers. A special case at the Hanford Site is the N Springs, which discharge low-level contamination (primarily ${ }^{90} \mathrm{Sr}$ ) into the Columbia River. These springs are perhaps more closely monitored than any comparable component of the Hanford Site ecosystem. Other means of diffuse emissions include erosion, uptake or intrusion of biota, and wind-caused resuspension.

4.3.1.15 Plutonium-Uranium Extraction Facility. The PUREX Plant is a collection of buildings and facilities located in the 200 East Area at the Hanford Site. The main building, 202-A, is a heavily shielded, reinforced concrete structure known as a canyon. This building contains the main equipment used in the PUREX process of chemically separating and purifying actinides from the irradiated nuclear fuel.

Radionuclides primarily associated with the PUREX Plant include ${ }^{14} \mathrm{C},{ }^{85} \mathrm{Kr},{ }^{90} \mathrm{Sr}$, ${ }^{106} \mathrm{Ru},{ }^{129} \mathrm{I},{ }^{137} \mathrm{Cs},{ }^{147} \mathrm{Pm},{ }^{238} \mathrm{Pu},{ }^{239,240} \mathrm{Pu},{ }^{241} \mathrm{Pu}$, and ${ }^{241} \mathrm{Am}$. Potential diffuse emission release points include access doors and the inlet-exhaust ventilation system, all of which are controlled. 
4.3.1.16 Uranium Oxide Plant. The Uraniun Oxide $\left(\mathrm{UO}_{3}\right)$ Plant, located in the 200 West Ârea, is used to produce $\mathrm{UO}_{3}$ powder by calcining uranyl nitrate solutions from the PUREX Plant. The $\mathrm{UO}_{3}$ powder is sealed in steel drums for shipment offsite. The $\mathrm{UO}_{3}$ Plant was on standby during 1992 but is expected to complete the processing of stored uranyl nitrate in 1993.

Uranium is the potential primary source of radioactive diffuse emissions from the $\mathrm{UO}_{3}$ Plant. Potential diffuse emission release points include access doors and the inletexhaust ventilation system, all of which are controlled.

4.3.1.17 Plutonium Finishing Plant. PFP was in standby during 1992. This facility is located in the 200 West Area and is used to recover, stabilize, and store plutonium. Recovered plutonium nitrate and plutonium nitrate so' utions received from the PUREX Plant are reduced to plutonium dioxide. The reduction process stabilizes the plutonium into the state best suited for long-term storage. The mission of PFP no longer includes the production of finished plutonium metal.

Radionuclides primarily associated with PFP include ${ }^{239,240} \mathrm{Pu},{ }^{241} \mathrm{Pu}$, and ${ }^{241} \mathrm{Am}$. Potential diffuse emission release points from PFP include access doors and the inlet-exhaust ventilation system, all of which are controlled.

4.3.1.17.1 234-5Z Building (active). The 234-5Z Building is also known as PFP or the 234-5 Building. The 234-5Z Building basement mostly consists of pipe tunnels carrying drain piping. The first floor houses the following: (1) two plutonium processing lines (Remote Mechanical A [RMA] and Remote Mechanical C [RMC] Lines) and their control rooms, (2) scrap stabilization gloveboxes, (3) plutonium storage vaults, and (4) the plutonium nitrate feed load-in/load-out ( $\mathrm{LI} / \mathrm{LO})$, blending, and storage facilities.

Radionuclides primarily associated with the $234-5 \mathrm{Z}$ Building include ${ }^{239,240} \mathrm{Pu}$ and ${ }^{241} \mathrm{Am}$. Potential diffuse release points include access doors and the inlet-exhaust ventilation system, all of which are controlled.

4.3.1.17.2 236-Z Building. The 236- $\mathrm{Z}$ Building, commonly known as PRF, is located south of the southeastern corner of the 234-5Z Building, connected to it by the 242-Z Building. The building air exhausts through the 291-Z-1 stack.

Radionuclides primarily associated with the $236-\mathrm{Z}$ Building include ${ }^{239,240} \mathrm{Pu}$ and ${ }^{241} \mathrm{Am}$. Potential diffuse release points include access doors and the inlet-exhaust vot til tion system, all of which are controlled.

4.3.1.17.3 232- $\mathrm{Z}$ Building. The 232-Z Building houses the layaway Contaminated Waste Recovery Process. It was commonly called the "Incinerator." It was constructed by Project CGC-013, "Plutonium Recovery from Contaminated Material." The Contaminated Waste Recovery Process was partially decontaminated and decommissioned in 1984. 
Radionuclides primarily associated with the $232-\mathrm{Z}$ Building include ${ }^{239,240} \mathrm{Pu}$ and ${ }^{241} \mathrm{Am}$. Potential diffuse emission release points include access doors and inlet-exhaust ventilation systems, all of which are controlled.

4.3.1.17.4 241- $Z$ and $242-Z$ Buildings. The $241-Z$ and $242-Z$ Buildings are designated as waste treatment facilities. $241-Z$ houses sumps, which are five separate enclosures or ventilated cells, each containing a 5,300-gal $(20,000-\mathrm{L})$ tank used to accumulate the liquid waste, generated by PFP before being transferred to the tank farms. The $241-Z$ is ventilated by the $296-Z-3$ stack.

The 242-Z Building houses abandoned waste treatment process equipment, once used to recover americium. This facility was permanently shut down after a process upset in 1979 spread contamination and caused irreparable equipment damage inside. The structural integrity of the facility was not compromised, however. The facility was then decontaminated extensively before being placed in layaway pending decommissioning work. The 242-Z Building shares the main ventilation system of the 234-5Z and 236-Z Buildings, exhausting its building air through the 291-Z-1 stack.

Radionuclides primarily associated with the $241-\mathrm{Z}$ and $242-\mathrm{Z}$ Buildings include ${ }^{239.240} \mathrm{Pu}$ and ${ }^{241} \mathrm{Am}$. Potential diffuse emission release points include access doors and the inletexhaust ventilation system, all of which are controlled.

4.3.1.17.5 2736-ZB Building. The 2736-ZB shipping area and receiving area both accommodate a maximum of 100 shipping containers, each of which is about the size of a 55-gal (210-L) drum. Adequate spacing is provided between containers to meet Westinghouse Hanford criticality pres ntion requirements, personnel exposure specifications, and corridor access standards to emergency staging areas. "The two areas are physically separated by a wall.

Radionuclides primarily associated with the $2736-\mathrm{ZB}$ Building include ${ }^{239,240} \mathrm{Pu}$ and ${ }^{241} \mathrm{Am}$. Potential diffuse emission release prints include access doors and the inlet-exhaust ventilation system, all of which are controlied.

4.3.1.17.6 216-Z-20 Crib Effluent Facility. The 216-Z-20 Crib was constructed and commissioned for use in "Aptember 1981 to dispose of waste water previously discharged to the 216-U-10 Pond through the 216-Z-19 Ditch. Use of the new crib allowed the radioactively contaminated 216-Z-19 Ditch and 216-U-10 Pond to be decommissioned.

Two liquid waste facilities were built. The $216-Z-20$ Crib was designed and constructed to dispose of potentially radioactive liquid effluents from the $231-Z, 232-Z$, 236-Z, 242-Z, 291-Z, 234-5Z, and 2736-ZB Buildings. The same building effluent headers and piping that served the 216-Z-19 Ditch were used. These headers and piping contain some residual contamination. The 207-Z Seepage Basin, later renamed 216-Z-21 Seepage Basin, was designed and constructed to dispose of noncontaminated water from the storm sewer north of the 234-5Z Building, water-tank overflow, and swamp-cooler waste water. 
The $216-\mathrm{Z}-20 \mathrm{Crib}$ is approximately $460 \mathrm{~m}(1,500 \mathrm{ft})$ long with a cross section at the

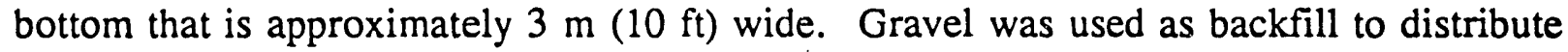
effluents throughout the crib. A vapor barrier was placed above the gravel backfill. Soil was placed over the top to bring the area back to the surrounding grade.

Radionuclides primarily associated with the $216-\mathrm{Z}-20$ Crib include ${ }^{239,240} \mathrm{Pu}$ and ${ }^{241} \mathrm{Am}$. Diffuse emissions may be released by way of vents, risers, erosion, biotic uptake and intrusion, and wind-caused resuspension.

4.3.1.18 T Plant. Originally, $T$ Plant was a fuel separations facility using the bismuth phosphate process. Now the facility is used for radioactive decontamination and repair of equipment. This facility currently is active.

T Plant is located in the 200 West Area of the Hanford Site. Buildings, structures, or special facilities included as part of this facility are the 221-T and 2706-T Buildings, deactivated 221-T Building Head-end, and 214-T Chemical Storage Building. Ancillary buildings, structures, and areas included are the 271-T, 291-T and 221-TA Buildings, and the 211-T Chemical Storage Area. Decontamination processes are conducted in the 221-T and 2706-T Buildings. The 221-T Building Head-end was used for special experiments and operations. The 214-T Building stores chemicals. The 211-T Chemical Storage Area consists of a 90-day permitted pad that stores nonradioactive hazardous waste and a tank that stores bulk chemicals. The 271-T Building provides office space to Westinghouse Hanford staff supporting $T$ Plant operations. The 291-T Building houses the exhaust ventilation fans for the 291-T-1 main stack. The 221-TA Building houses the supply ventilation fans for the 221-T Building canyon.

Radioactive decontamination activities are performed in the canyon area. The canyon area consists of 37 cells and one railroad tunnel door. The railroad tunnel (used for transporting equipment into and out of the canyon, as well as for some decontamination) enters the plant at cell $2 \mathrm{~L}$. A motor-driven rolling steel door, provides railroad canyon access.

Primary radionuclides associated with $\mathrm{T}$ Plant include ${ }^{90} \mathrm{Sr},{ }^{137} \mathrm{Cs}$, and ${ }^{239.240} \mathrm{Pu}$. Potential diffuse emission release points include access doors and the inlet-exhaust ventilation system, all of which are controlled.

4.3.1.18.1 2706-T Building. The 2706-T Building is a ground-level structure constructed of prefabricated steel. It has two openings on the west end that are fitted with rollup metal doors that allow access to the pit areas. Low-level radioactive decontamination activities are performed in these two pits.

Radionuclides primarily associated with the 2706 - T Building include ${ }^{90} \mathrm{Sr},{ }^{137} \mathrm{Cs}$, and ${ }^{239.240} \mathrm{Pu}$. Potential diffuse emission release points include access doors and the inlet-exhaust ventilation system, all of which are controlled. 
4.3.1.18.2 221-T Building - Head-End Operations. The 221-T Building head-end consists of a canyon area extending from the basement floor to the roof. This canyon area has several deck levels and a parapet wall. Four floor levels adjacent to the canyon house include: (1) an electrical switchgear room, (2) a chemistry laboratory, (3) office areas, (4) a change room, (5) a lunch room, (6) a control room, (7) an instrument shop, (8) a maintenance shop, (9) and storage areas. Flow from eight process sewer lines originating in the Head-End is discharged to the 216-T-1 Ditch.

Radionuclides primarily associated with 221 -T Head-End Operations include ${ }^{90} \mathrm{Sr}$, ${ }^{137} \mathrm{Cs}$, and ${ }^{239,240} \mathrm{Pu}$. Potential diffuse emission release points include access doors and inletexhaust ventilation system, all of which are controlled.

\subsection{1-T Building - Pressurized-Water (Cooled) Reactor Fuel Assembly} Storage. Canyon cell $2 \mathrm{R}$ has been modified to store approximately 76 pressurized-water reactor Core 2 blanket fuel assemblies that were used to power DOE's Shippingport Reactor. Provisions have been made to store the assemblies at the Hanford Site for up to 20 years.

The 221-T Building galleries are maintained at atmospheric pressure, while the 221-T Building canyon area is maintained at a negative pressure with respect to atmosphere. A primary design feature of these systems is to ensure that potentially contaminated canyon air is completely separate from the clean air in the 221-T Building galleries and the 271-T Building, which is the office space connected to the 221-T Building. In addition, the canyon air system is operated at negative pressures with respect to the other systems.

The 271-T building is adjacent to the 221-T Operating Gallery. While most of this building is used for office space, portions are used by T-Plant Operations.

Radionuclides primarily associated with 221-T-PWR Fuel Assembly Storage include ${ }^{90} \mathrm{Sr},{ }^{137} \mathrm{Cs}$, and ${ }^{239,240} \mathrm{Pu}$. Potential diffuse emission release points include access doors and the inlet-exhaust ventilation system, all of which are controlled.

4.3.1.18.4 Transuranic Waste Storage and Assay Facility. Originally, the function of the 224-T Building was to purify plutonium nitrate by the lanthanum-fluoride process. The plant remained inactive following phase-out of the bismuth phosphate plants until the early 1970s. At that time, the building was modified for storage of plutonium scrap in liquid and solid forms.

In 1984, the 224-T Building was chosen to house the transuranic (TRU) waste storage and assay operation. The Transuranic Waste Storage and Assay Facility (TRUSAF) operation consists of a nondestructive analysis of TRU waste. The analysis is used as an overview for sealed, certified, contact-handled, TRU solid-waste packages, to verify general compliance with the Waste Isolation Pilot Plant (WIPP) Waste Acceptance Criteria (WAC). Those containers meeting WIPP WAC criteria are stored at 224-T and maintained in a manner to retain their certification pending shipment to WIPP. The TRUSAF operation also performs a sorting function for the PFP. 
In 1985 , the removal of plutonium scrap from $224-\mathrm{T}$ was completed, and the building was officially designated as the TRUSAF.

Radionuclides primarily associated with TRUSAF include ${ }^{90} \mathrm{Sr},{ }^{137} \mathrm{Cs}$, and ${ }^{239,240} \mathrm{Pu}$. Potential diffuse emission release points include access doors and the inlet-exhaust ventilation system, all of which are controlled.

4.3.1.19 B Plant. B Plant was originally designed to chemically process spent nuclear fuels. Radiological containment and confinement features were incorporated in the various facilities and support systems to prevent exposure of plant personnel and the general public to excessive radiation. The plant was then modified to separate strontium and cesium from the fission product waste stream following plutonium and uranium recovery from irradiated reactor fuels in the PUREX Plant. The recovered purified and concentrated strontium and cesium solutions were then transferred to the WESF for conversion to solid compounds, encapsulation, and interim storage. After strontium and cesium removal, the remaining waste was transferred from B Plant to the tank farms.

B Plant is an operating facility used to ensure safe storage and management of the WESF cesium and strontium capsules and a radiological inventory remaining in the plant from previous campaigns. There are currently no production activities at B Plant, but several systems are operating systems to perform the current B Plant mission. B Plant receives and stores various chemicals from: (1) commercial suppliers for treatment of lowlevel waste generated at WESF and B Plant, (2) generation of demineralized water, (3) and conditioning of water used in HVAC units.

B Plant consists of three main buildings. They are the 221-B Processing Building, 271-B Service and Office Building, and 225-B Building (WESF). The 221-B Process Building and its attached 271-B Service Building were constructed in 1943. Construction of the WESF was completed in 1974.

Radionuclides primarily associated with B Plant include ${ }^{90} \mathrm{Sr}$ and ${ }^{137} \mathrm{Cs}$. Potential diffuse emission release points include access doors and the inlet-exhaust ventilation system, all of which are controlled.

4.3.1.20 222-S Facility. The 222-S Laboratory Complex is located in the southeast corner of the 200 West Area. The facility is composed of the main laboratory complex (222-S) and a number of ancillary buildings and structures.

4.3.1.20.1 222-S Laboratory. The 222-S Laboratory is a twu-story, aboveground building with a subterranean service level. This building is divided into laboratory support spaces, office spaces, a multicurie wing, and supplemental service areas. The building is designed with its own waste disposal facility, decontamination facility, fire protection and alarm system, ventilation system, and radiation monitoring systems. 
The 222-S Laboratory Annex houses the maintenance shop, instrument shop, and the counting room filter building.

Radionuclides primarily associated with the $222-\mathrm{S}$ Laboratory include ${ }^{90} \mathrm{Sr}$ and ${ }^{137} \mathrm{Cs}$. Potential diffuse emission release points include access doors and the inlet-exhaust ventilation system, all of which are controlled.

4.3.1.20.2 222-SA Laboratory. The 2 22 -SA Laboratory is a five-wide modular building located southeast of the $22<-\mathrm{s}$ Laboratory. Part of this laboratory prepares nonradioactive standards for Hanford Site laboratories. The other section of the laboratory is used for cold-process development work and standards preparation. The 222-SA Laboratory only has the potential for nonradioactive diffuse emissions.

4.3.1.20.3 2716-S Storage Building. The 2716-S Storage Building, located south of the 222-S Laboratory, is partitioned off for the storage of acids and bases. It provides both long- and short-term storage capability for laboratory materials and contains no radioactive materials.

There are no radioactive diffuse emissions from the 2716-S Storage Building.

4.3.1.20.4 207-SL Retention Basin. The 207-SL Retention Basin acts as a temporary holding facility for potentially radioactive or hazardous liquid effluents before discharge to the 216-S-26 Crib. Waste water from the laboratory, normally free of radioactive and hazardous chemical contamination, is routed to the 207-SL Retention Basin. This facility is a covered, below-grade concrete structure, directly east of the 222-S Building. Two 25,000-gal (95,000-L) compartments allow batch collection, sampling, and discharge of the waste.

Radionuclides primarily associated with the 207-SL Retention Basin include ${ }^{90} \mathrm{Sr}$ and ${ }^{137} \mathrm{Cs}$. Potential diffuse release points include access doors and seams in the basin cover blocks.

4.3.1.20.5 219-S Waste Handling Facility. The 219-S Waste Handling Facility collects liquid waste generated by the 222-S Laboratory operations that is contaminated radioactively and/or with hazardous chemicals. This facility consists of two below-grade vaults ( $A$ and $B$, also called cells); a below-grade concrete vault, containing three stainlesssteel tanks; a transite building; the pipe trench and operating gallery; and an ?.ttached concrete-walled sample gallery. Tanks TK-101 and TK-102 are in vault A and tank TK-103 is in vault $B$.

Radionuclides primarily associated with the 219-S Waste Handling Facility include ${ }^{90} \mathrm{Sr}$ and ${ }^{137} \mathrm{Cs}$. Potentiai diffuse release points include access doors and seams in the vault cover blocks. 
4.3.1.20.6 222-SC Filter Building. The 222-SC Filter Building contains the filtration for hot-cells 1-A, 1-E-1, 1-E-2, and 1-F. The hot-cells in rooms 1-A, 1-E, and 1-F are serviced by the main building supply and exhaust ventilation, but additional features are present to increase safety and containment.

Radionuclides primarily associated with the 222-SC Filter Building include ${ }^{90} \mathrm{Sr}$ and ${ }^{137}$ Cs. Potential diffuse emission release points include access doors and the inlet-exhaust ventilation system, all of which are controlled.

4.3.1.20.7 222-SB Filter Building. The 222-SB Filter Building houses 96 singlestage HEPA filters to provide final filtration for the 222-S Laboratory. Under normal operation of the ventilation system, two fans exhaust air from the laboratory. Exhaust air leaves the 222-S Building through the 296-S-21 stack. A third exhaust fan, driven by a diesel engine, will provide half of the normal operation of the exhaust system if the electrically powered fans fail.

Radionuclides primarily associated with the 222-SB Filter Building include ${ }^{90} \mathrm{Sr}$ and ${ }^{137}$ Cs. Potential diffuse emission release points include access doors and the inlet-exhaust ventilation system, all of which are controlled.

4.3.1.20.8 218-W-7 Dry Waste Burial Vault. The 218-W-7 Dry Waste Burial Vault is located $40 \mathrm{ft}$ southeast of 222-S. This underground concrete vault was removed from service sometime around 1960. It was used primarily for disposal of plutonium-contaminated dry-hood waste generated by the 222-S Laboratory. Access to the tank is through a locked hatchway.

Plutonium-239 and -240 are the radionuclides primarily associated with the 218-W-7 Dry Waste Burial Vault. A locked access hatchway is the only potential release point for diffuse emissions.

4.3.1.20.9 216-S-26 Crib. The 216-S-26 Crib receives all waste water collected in the 207-SL Retention Basiris that meets radiological and chemical limits. Additionally, the crib also receives 222-SA Laboratory waste water and steam condensate from the $291-S$ Stack Fan House, which bypasses the 207-SL Retention Basins.

Radionuclides primarily associated with the $216-\mathrm{S}-26 \mathrm{Crib}$ include ${ }^{90} \mathrm{Sr},{ }^{137} \mathrm{Cs}$, and ${ }^{239,240} \mathrm{Pu}$. Diffuse emissions may be released by way of vents, risers, erosion, biotic uptake and intrusion, and wind-caused resuspension.

4.3.1.21 2724-W Protective Equiprnent Decontamination Facility. The 2724-W Protective Equipment Decontamination Facility (PEDF) is located in the 200 West Area of the Hanford Site. The original laundry building is a wood-and-concrete structure, constructed in 1952. It has been expandet throughout its history using prefabricated metal buildings and mobile offices. 
The current complex is on one level and encompasses these connected buildings: 2724-W, 2724-WA, 2724-WB, and MO-406. There are separate process areas for radioactive and for nonradioactive washing and drying in Building 2724-W. The remaining buildings are for laundry finishing tasks, storage, change rooms, offices, and lunchroom. Mask cleaning and repair operations are performed in a mobile office complex (MO-412), known as the "mask station," which is adjacent to the laundry facility.

Radionuclides primarily associated with the $2724-\mathrm{W}$ PEDF include ${ }^{90} \mathrm{Sr}$ and ${ }^{137} \mathrm{Cs}$. Potential diffuse emission release points include access doors and the inlet-exhaust ventilation system, all of which are controlled.

4.3.1.22 Solid Waste Compactor Facility. The Solid Waste Compactor Facility, the 213W Building, is located in the 200 West Area of the Hanford Site. The 213-W Building is adjacent to the 272-WA Building (the Operations Support Building) at the 218-W-5 Burial Grounds at the west end of the 200 West Area. Systems ancillary to the 213-W Building also are included.

The primary function or process associated with the Solid Waste Compactor Facility is compacting and packaging dry, low-level radioactive waste for disposal. The facility is currently in active operation.

Radionuclides primarily associated with the $213-\mathrm{W}$ Building include ${ }^{90} \mathrm{Sr}$ and ${ }^{137} \mathrm{Cs}$; low-level waste is present also. Potential diffuse emission release points include access doors and the inlet-exhaust ventilation system, all of which are controlled.

4.3.1.23 Central Waste Complex. The Central Waste Complex (CWC) is a group of structures located on the west side of 200 West Area exclusion zone. The primary function or process associated with the CWC Facility is the receipt and storage of radioactive and mixed waste (MW), which is currently ongoing. This facility has the potential to generate radioactive and/or hazardous chemical emissions and radioactive and/or hazardous chemical liquid effluent.

Radionuclides associated with the Central Waste Complex are from a wide group of mixed fission, mixed waste, and transuranic radionuclides. Potential diffuse emission release points include access doors and the inlet-exhaust ventilation system, all of which are controlled. The facility consists of the following.

4.3.1.23.1 Pu and PCE Storage Facility. This facility is a steel building with concrete curbing within its perimeter. The building has a water-based fire suppression system and CAMs for detection of airborne radioactive particulates.

4.3.1.23.2 Low-Flashpoint MW Storage Modules. Each of the low-flashpoint IMW storage modules has a catch sump with a holding capacity of $750 \mathrm{gal}(2,800 \mathrm{~L})$. Modules used for storage of TRU-mixed waste also have draft ventilation. 
4.3.1.23.3 Mixed-Waste Storage Facilities. The buildings 2402-WB through 2402-WL, 2402-W, and 2403-W are metal structures with concrete floors, ventilation systems, and water-based fire protection. The concrete floors have provisions to collect any spilled liquids. The facilities have CAMs for detection of airborne radioactive particulates.

4.3.1.23.4 Mixed-Waste Storage Pad. The MW Storage Pad is a $9,000 \mathrm{ft}^{2}\left(320 \mathrm{~m}^{3)}\right.$ concrete storage pad with 6 -in. $(15-\mathrm{cm})$ curbing around its perimeter. The pad has a stormwater collection system that allows for disposing of the stormwater after sampling and analysis.

\subsection{Waste Receiving and Storage Pad. The Waste Receiving and Storage} Pad is a 61-m-by-46-m (200-ft-by-150-ft) asphalt pad.

4.3.1.24 Tank Farms. Liquid waste from chemical processing operations containing high concentrations of radionuclides is stored on an interim basis in underground tanks. The Hanford Site tank farms contain 177 tanks (149 SSTs and 28 DSTs) with capacities ranging from 50,000 to 1.2 million gal (190,000 to 4.5 million L). Since 1967, newly generated liquid waste has been stored in DSTs. The SSTs are no longer receiving waste.

The location of all the tank farms is the 200 East and 200 West Areas. Both DSTs and SSTs are present in these areas. Tank farms in the 200 East Area include the A, AX, B, BX, BY, C (SSTs), AN, AZ, AY, AP, and AW (DSTs). Those in the 200 West Area are the S, SX, T, TX, TY, U (SSTs) and the SY (DST).

Hanford Site tank farms comprise transfer routes, diversion boxes, storage vaults, double-contained receiver tanks (DCRT), and evaporators.

A system of underground pipes is used to transfer wastes from the 200 East Area waste generators to the DSTs, between the DSTs, and from the DSTs to treatment and storage units in the 200 East and 200 West Areas.

Radionuclides primarily associated with the tank farms include ${ }^{3} \mathrm{H},{ }^{14} \mathrm{C},{ }^{90} \mathrm{Sr},{ }^{106} \mathrm{Ru}$, ${ }^{125} \mathrm{Sb},{ }^{137} \mathrm{Cs},{ }^{239,240} \mathrm{Pu}$, and ${ }^{241} \mathrm{Am}$. Diffuse emission release locations may include vents, risers, access hole covers, inlet-exhaust ventilation systems, diversion boxes, transfer lines, and storage vaults.

4.3.1.24.1 Double-Shell Waste Tanks. The DSTs are of two distinctly different types. The capacity of the first type is 1 to 1.2 million gal (3.79 to 4.54 million $\mathrm{L}$ ), and is designed for long-term storage (up to 50 years) of high-activity mixed waste. Twentyfour 1.2-million-gal (4.5-million-L) non-aging waste DSTs and four 1.0-million-gal (3.8-million-L) aging waste DSTs have been built. For efficiency during construction and operation, the 1-million-gal tanks were grouped into six tank farms.

The second type of of DST is smaller, with storage capacities ranging from 800 to $45,000 \mathrm{gal}(3,028$ to $170,370 \mathrm{~L})$. These tanks were used primarily for lag storage of waste 
before transfer to the larger tanks or to other facilities. These smaller tanks are called DCRTs.

All DSTs were fabricated as three concentric tanks. Waste is stored in the freestanding primary tank. The secondary tank sits on a concrete pad. The completely enclosed annulus serves as a containment barrier if the primary tank should leak. The annulus is ventilated and continually monitored for evidence of primary tank leakage. The third tank is a concrete shell that encloses the sides of both primary and secondary tanks for additional containment, radiation shielding, and structural support.

Ancillary equipment also is present, such as transfer lines between tank farms and DCRTs, valve pits, diversion boxes, and tank-farm piping.

4.3.1.25 242-A Evaporator. The 242-A Evaporator complex is located in the 200 East Area of the Hanford Site. The 242-A Building is located south of the 241-A and 241-AX Tank Farms and north of the 242-AW Tank Farm. The 242-A Building contains the evaporator vessel and supporting process equipment. The building ventilation exhaust fans and HEPA filter housings are located on the north side of the building. An emergency diesel generator is located on the south side of the building. Raw water, steam, and electrical power are provided to the 242-A Building from existing service facilities in the 200 East Area.

In general, the 242-A Evaporator Facility can be divided into three areas: process, service, and operating. The process area includes: the evaporator room, pump room, condenser room, and ion exchange enclosure. The service area includes: the aqueous make-up (AMU) room, loadout and hot equipment storage room, loading room, and HVAC room. The operating areas include: the control room, personnel change rooms, lunchroom, office, and storage rooms.

Radionuclides primarily associated with 242 -A include ${ }^{3} \mathrm{H},{ }^{14} \mathrm{C},{ }^{90} \mathrm{Sr},{ }^{106} \mathrm{Ru},{ }^{125} \mathrm{Sb},{ }^{137} \mathrm{Cs}$, ${ }^{239,240} \mathrm{Pu}$, and ${ }^{241} \mathrm{Am}$. Potential diffuse emission release points include access doors and the inlet-exhaust ventilation system, all of which are controlled.

4.3.1.26 242-S Evaporator. The 242-S Evaporator, currently on standby, is located in the 200 West Area, west of the 241-SY Tank Farm. It consists of an evaporator vessel and supporting process equipment. The building ventilation exlaaust fans and HEPA filter housings are on the north side of the building. An emergency diesel generator is on the south side of the building. Raw water, steam, and electrical power are provided from existing service facilities in the 200 East Area. There are three main areas: process, service, and operations. The process area includes: the evaporator room, pump room, condenser room, and ion-exchange enclosure. The service area includes: the Aqueous Make-up (AMU) room, loadout and hot equ: ment storage room, loading room, and the HVAC room. The operating areas include: the control room, personnel change rooms, lunchroom, offices, and storage rooms. 
Radionuclides primarily associated with $242-\mathrm{S}$ include ${ }^{3} \mathrm{H},{ }^{14} \mathrm{C},{ }^{90} \mathrm{Sr},{ }^{106} \mathrm{Ru},{ }^{125} \mathrm{Sb},{ }^{129} \mathrm{I}$, ${ }^{137} \mathrm{Cs}$, ${ }^{239,240} \mathrm{Pu}$, and ${ }^{241} \mathrm{Am}$. Potential diffuse emission release points include access doors and the inlet-exhaust ventilation system, all of which are controlled.

4.3.1.27 242-T Evaporator. The 242-T Evaporator, currently inactive, is located in the 200 West Area, east of the 241-TX and 241-TY Tank Farms. It consists of evaporator vessel and supporting process equipment. The building ventilation exhaust fans and HEPAfilter housings are on the north side of the building. An emergency diesel generator is on the south side of the builidng. Raw water, steam, and electrical power are provided from existing service facilities in the 200 East Area. There are three main areas: process, service, and operations. The process area includes: the evaporator room, pump room, condenser room, and ion-exchange enclosure. The service area includes: the AMU room, loadout and hot equipment storage room, loading room, and the HVAC room. The operating areas include: the control room, personnel change rooms, lunchroom, offices, and storage rooms.

4.3.1.28 Grout Treatment Facility. The GTF combines low-level radioactive liquid waste with a cement mixture that is pumped into disposal vaults. The GTF was inactive during 1992.

Radioactive constituents primarily associated with GTF include ${ }^{90} \mathrm{Sr},{ }^{137} \mathrm{Cs}$, and other low-ievel radioactive waste from the tank farms. Potential diffuse emission release modes include vent, risers, and the inlet-exhaust ventilation system.

4.3.1.29 Low-Level Burial Grounds. The active low-level radioactive solid waste burial grounds are located in the 200 Areas of the Hanford Site. The active sites to be considered are those addressed in the Part A, Dangerous Waste Permit Application for the Low-Level Burial Grounds (DOE/RL 1988). In the 200 West Area, the burial grounds to be considered include 218-W-5, 218-W-6, 218-W-3A, 218-W-3AE, 218-W-4B, and 218-W-4C. In the 200 East Area, the burial grounds to be considered include 218-E-10 (Expansion), 218-E10, and 218-E12B.

The entire array of low-level radionuclides from operations on the Hanford Site is associated with the Low-Level Burial Grounds. Diffuse emissions may be released by way of open burials (before covering), ruptured containment vessels, biotic intrusion, erosion, and wind-caused resuspension.

4.3.1.29.1 Burial Ground 218-W-3A. Burial Ground 218-W-3A began receiving waste in 1970. It consists of 61 trenches covering 50.3 acres (20.4 hectares). Waste stored or disposed of in this burial ground includes mixed, transuranic, low-level, and retrievable waste. Other types of waste include ion-exchange resins and industrial waste (such as failed equipment, tanks, pumps, ovens, agitators, heaters, hoods, jumpers, vehicles, and accessories). This burial ground also stores spent fuel. 
4.3.1.29.2 Burial Ground 218-W-3AE. Burial Ground 218-W-3AE began receiving waste in 1981. It consists of 31 trenches covering 49.4 acres (20 hectares). Waste in this burial ground includes low-level and mixed waste. Other types of waste placed in this burial ground include rags, paper, rubber gloves, disposable supplies, broken tools, and industrial waste.

4.3.1.29.3 Burial Ground 218-W-4B. Burial Ground 218-W-4B began receiving waste in 1968. It consists of 13 trenches and 12 caissons covering 8.6 acres ( 3.5 hectares). The trenches contain mixed and retrievable TRU waste and were filled before 1980. Caisson Alpha 4 is believed to contain mixed waste.

4.3.1.29.4 Burial Ground 218-W-AC. Burial Ground 218-W-4C began receiving waste in 1978. It consists of 69 trenches covering 51.7 acres (20.9 hectares). Some of the Wa-te in this facility is TRU, mixed, and low-level. Other types of waste placed in these trenches include contaminated soil, decommissioned pumps, pressure vessels and hardware, and stored spent fuel. Some of the trenches are designed for retrievable storage of waste.

4.3.1.29.5 Burial Ground 218-W-5. Burial Ground 218-W-5 began receiving waste in 1986. It consists of 35 trenches (with room to expand to 57 trenches) covering 84.0 acres (34 hectares). The trenches contain low-level mixed waste that includes lead bricks and shielding. Low-level waste also was placed in these trenches.

4.3.1.29.6 Burial Ground 218-W-6. Burial Ground 218-W-6 has not received any waste to date. When developed, this burial ground will consist of 35 trenches and cover approximately 44.5 acres (18 hectares).

4.3.1.29.7 Burial Ground 218-E-10. Burial Ground 218-E-10 began receiving waste in 1960. It consists of 18 trenches covering 56.7 acres (22.9 hectares). Waste was received from PUREX, B Plant, and N Reactor. The waste includes low-level and low-level mixed waste such as dragoff waste, failed equipment, and industrial waste.

4.3.1.29.8 Burial Ground 218-E-12B. Burial Ground 218-E-12B began receiving wastes in 1967. It consists of 94 trenches covering about 173 acres ( 70 hectares). Areas are set aside for future expansion of this burial ground. Some of the waste contained in this burial ground is TRU, mixed, and low-level. Trench 94 contains decommissioned U.S. Navy submarine reactor compartments.

4.3.1.30 300 Area Waste Service Facility. The 340 Complex is located in the 300 Area of the Hanford Site near the Columbia River. The 340 Complex was constructed to collect, store, and transport radioactive liquid wastes. Buildings $324,325,326,327$, and 329 were built in the mid-1950's and can discharge liquid radioartive waste to the Radioactive Liquid Waste System or potentially radioactive wastes to the Retention Process Sewer (RPS). The 308 building can discharge wastes only to the RPS. Except for the 308 building, which is managed by Westinghouse Hanford for RL, PNL manages all facilities generating waste 
destined for the 340 Complex. Only the 324 and 325 buildings can accumulate wastes in tanks and then pump the wastes to the complex.

Uranium, ${ }^{60} \mathrm{Co}$, and ${ }^{90} \mathrm{Sr}$ primarily are associated with the 340 Complex. Potential diffuse emission release points include access doors and the inlet-exhaust ventilation system, all of which are controlled.

\subsubsection{300 Area N Reactor Fuels Fabrication Facility. The N Reactor Fuels} Fabrication Facility is located in the 300 Area of the Hanford Site. It consists of 16 buildings, two tank farms, and associated pipe trenches and drains. The structures are located on the north side of the 300 Area. The 300 Area Fuels Fabrication facilities began operation in 1944 with some structures being added in the 1950's and 1960's. The following sections provide information on each of the facilities.

4.3.1.31.1 333 Building. The primary N Reactor Fuels Fabrication activities were conducted in the 333 Building. The fuel fabrication operation produced $\mathrm{N}$ Reactor fuel from 1961 until 1987 when operations were stopped.

This facility used a variety of mechanical, chemical, and electrical processes to convert uranium billets and assorted components into finished fuel assemblies for irradiation at $\mathrm{N}$ Reactor.

Operations and resulting radionuclide air emissions in the 333 Building ceased in January 1987. Emission monitoring equipment was shut down at this time. Process liquid effluents also have stopped; however, steam condensate water, air conditioning water, and stormwater still discharge to the process trench by way of the process sewer.

Uranium and ${ }^{99} \mathrm{Tc}$ primarily are associated with the 333 Euilding. Potential diffuse emission release points include access doors and the inlet-exhaust ventilation system, all of which are controlled.

4.3.1.31.2 313 Building. The 313 Building (N Fuels Fabrication Support Facility) houses: the uranium laboratory, copper casting, waste-acid treatment facility, extrusion press, engineering development laboratory, training rooms, and staff offices.

The 313 Building was used from 1944 to 1971 for fabricating uranium fuel elements for the eight retired, single-pass reactors in the 100 Areas. The function of the building changed when those reactors ceased operating. The building then was used as an N Fuels Fabrication Support Facility to treat uranium-bearing acid in the waste-acid treatment system. The recovered uranium sludge was recycled to form new uranium billets at Fernald, Ohio.

Uranium is the potential primary radioactive constituent in diffuse emissions from the 313 Building. The potential pitmary diffuse emission release poirts include personnel and equipment access doors and the inlet-exhaust ventilation system, all of which are controlled. 
4.3.1.31.3 303-K Radioactive Mixed Waste Storage Facility. The 303-K Facility was designed and constructed in 1943. The facility is a concrete-block building with a poured concrete ceiling. Outside, the storage area consists of two concrete pads, two asphalt pads, and a gravel area. The north room of the 303-K Facility originally had one roof exhaust fan. The fan was used from 1953 to 1977 while aluminum spacer and equipment decontaminating was done. The roof vent fan was replaced with a HEPA-filtered exhaust system in 1977, which was used until the fall of 1982. The exhaust system was only turned on at the end of the curing operation for the concreted billets of recyclable scrap uranium chips and fines, or if hydrogen levels indicated a billet fire had occurred. The HEPA exhaust system has not operated since the concrete curing operation was discontinued in 1982. The facility no longer discharges either air emissions or liquid effluents to the environment. It has been used to store radioactive and mixed waste since 1982.

Uranium is the potential primary radioactive constituent in diffuse emissions from the 303-K Facility. The potential primary diffuse emission release points include personnel and equipment access doors, which are controlled.

4.3.1.31.4 303-M Uranium Oxide Facility. The 303-M Uranium Oxide Facility is adjacent to the 333 Building and consists of one building, and an adjacent outdoor drum storage area, and a small process sewer filter building. The 303-M Uranium Oxide Facility stored and treated recycled material generated during the $\mathrm{N}$ Reactor fuel fabrication processes. The fuel fabrication waste material consisted of pyrophoric saw fines and lathe turnings, known as chips, composed of slightly enriched uranium and Zircaloy-2. The wastes were calcinated to remove their pyrophoric properties, which eliminated the possibility of spontaneous combustion while being transported.

The 303-M Uranium Oxide Facility operated from 1983 to 1987 for the calcination of wastes generated by Westinghouse Hanford and previous operators. The facility ceased operating in 1987.

Uranium is the potential primary radioactive constituent in diffuse emissions from the 303-M Facility. The potential primary diffuse emission release points include personnel and equipment access doors and the inlet-exhaust ventilation system, all of which are controlled.

4.3.1.31.5 304 Uranium Concretion Facility. The 304 Building was designed and constructed in 1952 as a pilot plant for the lead-dip canning process. The 304 Uranium Concretion Facility was installed in 1971 with a drainage trench and sump to remove liquids resulting from spills, leaks, and daily operations. Standard spill-response procedures inside the building included washing the spilled waste to the sump where the fines would settle out. The vaste water was drained into the 300 Area process sewer, and the fines were shoveled from the sump and concreted. Additionally, an outside storage area is on the north side of the facility. The storage area is a concrete pad surrounded by asphalt. 


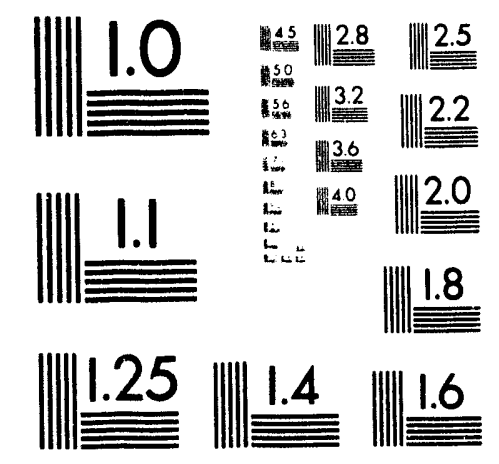



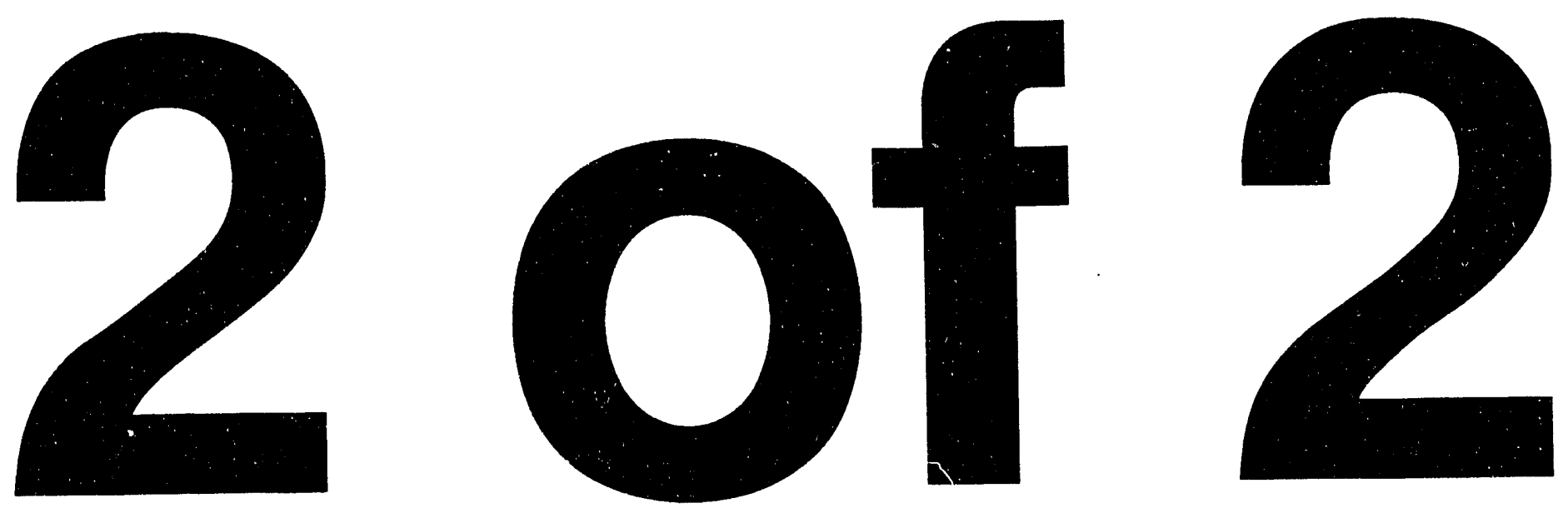
Uranium is the potential primary radioactive constituent in diffuse emissions from the 304 Facility. The potential primary diffuse emission release points include personnel and equipment access doors and the inlet-exhaust ventilation system, all of which are controlled.

4.3.1.31.6 311 Tank Farm. The recoverable and nonrecoverable uranium-bearing neutralized waste solutions from the 313 Building were combined in aboveground tanks (tanks 40 and 50) in the 311 Tank Farm. These liquid wastes eventually were transferred to tanker trucks and disposed of in the 183-H Basins or the 200 Areas. After 1988, if the wastes were below radioactive release limits, they were shipped to offsite contractors for treatment, storage, and/or disposal. The two tanks received approximately $420,000 \mathrm{gal}$ (1.6 million $\mathrm{L}$ ) of waste per year during periods of peak production.

In addition to waste tanks 40 and 50, the 311 Tank Farm has a 4,000-gal $(15,000-\mathrm{L})$ tank that was used to store nitric acid and two 10,000-gal $(38,000-\mathrm{L})$ tanks that were used to store sodium hydroxide. The tanks were emptied in 1991 and no longer contain nitric acid or sodium hydroxide.

Uranium is the potential primary radioactive constituent in diffuse emissions from the 311 Tank Farm. Diffuse emissions may be released by way of uranium-bearing material transfers and unplanned releases.

\subsection{4-A Waste Acid Storage Building. The 334-A Waste Acid Storage} Building was completed in late 1974 and placed in use in January 1975 . For four months in 1973, an underground tank and tank 4 in the 334 Tank Farm were used to collect acid waste that would be transferred to the 313 Building for neutralization. The underground tank began to leak in August 1973 and was removed during construction of the 334-A Building. The waste acids were discharged directly into the process sewer until the 334-A Building was built in December 1974. The tanks in the 334-A building received approximately $210,000 \mathrm{gal}(790,000 \mathrm{~L})$ of waste acids per year. These waste acids consisted of hydrofluoric, nitric, and sulfuric acids with copper, zirconium, chromium, and uranium in solution. Following storage, the acids were pumped from the 334-A Building to the south end of the 313 Building for neutralization.

Uranium is the potential primary radioactive constituent in diffuse emissions from the 334-A Building. The potential primary diffuse emission release points include personnel and equipment access doors and the inlet-exhaust ventilation system, all of which are controlled.

4.3.1.32 Fast Flux Test Facility. The FFTF is a 400 -megawatt-thermal, sodium-cooled, fast-neutron-flux reactor designed specifically for irradiation testing of nuclear reactor fuels and materials for liquid-metal, fast-breeder reactors. The reactor is capable of extensive incore irradiation testing, including eight core positions that may be used with independent instrumentation for the test loops. In addition to irradiation-testing capabilities, FFTF is designed for long-term testing and evaluation of plant components and systems for liquidmetal, fast-breeder reactors. 
The FFTF is a complex of buildings and equipment arranged around the reactor containment building. This arrangement includes the reactor, equipment, and structures for heat removal, containment, core component handling and examination, instrumentation and control, and buildings for supplying utilities and other essential services.

Radionuclides primarily associated with FFTF include ${ }^{41} \mathrm{Ar},{ }^{85} \mathrm{Kr},{ }^{90} \mathrm{Sr}$, and ${ }^{131} \mathrm{I}$. The potential primary diffuse emission release points include the personnel and equipment access doors and the inlet-exhaust ventilation system, all of which are controlled. 
DOE/RL-93-36

This page intentionally left blank. 


\subsection{SUPPLEMENTAL INFORMATION}

This section has supplemental information related to Hanford Site radionuclide air emissions in 1992. The information consists of:

- Population dose estimate

- Compliance status with Subparts Q and T of 40 CFR 61 (EPA 1992)

- Discussion on ${ }^{220} \mathrm{Rn}$ emissions

- Emission points subject to monitoring requirements of 40 CFR 61.93(b) and costs to upgrade noncompliant monitoring systems

- Periodic confirmatory measurement plans

- Status of quality assurance (QA) program intended to comply with description in 40 CFR 61, Appendix B, Method 114.

\subsection{POPULATION DOSE}

The regional dose from 1992 Hanford Site air emissions was estimated by calculating the radiation dose to the population of approximately 376,000 residing within an $80 \cdot \mathrm{km}$ $(50-\mathrm{mi})$ radius of the onsite operating areas. Pathways of exposure to the population from releases of radionuclides to the atmosphere include inhalation, air submersion, and consumption of food. The collective effective dose equivalent (using the GENII computer code, Napier et al. 1988) to the population was 0.6 person-rem in 1992.

\subsection{COMPLIANCE STATUS WITH SUBPARTS Q AND T OF 40 CFR 61}

In 40 CFR 61 (EPA 1992), Subpart Q, "National Emission Standards for Radon Facilities From Department of Energy Facilities," paragraph 61.190 states that the provisions of Subpart $Q$ apply to the design and operation of all storage and disposal facilities for radium-bearing material that emit ${ }^{222} \mathrm{Rn}$ to the air. Paragraph 61.191(b) states that a source means any building, structure, pile, impoundment, or area used for interim storage or disposal that is or contains waste material containing radium in :jufficient concentration to emit ${ }^{222} \mathrm{Rn}$ in excess of the $20 \mathrm{pCi} / \mathrm{m}^{2} / \mathrm{s}$. At the Hanford Site, no storage and dispos $2 \mathrm{l}$ facilities for radium-bearing waste materials are established. Therefore, the provisions of this subpart are not applicable to the Hanford Site.

Activities at the Hanford Site were evaluated for compliance with 40 CFR 61 Subpart T, "National Emissions Standards for Radon Emissions From the Disposal of 
Uranium Mill Tailings" (EPA 1992). In paragraph 61.220, "Designation of Facilities," owners and operators of all sites used for the disposal of tailings and that managed residual radioactive material or uranium byproduct materials during and following the processing of uranium ores and that are listed in or designated by the Secretary of Energy under Title I of the Uranium Mill Tailings Control Act of 1978 or regulated under Title II of that act are subject to the provisions of the subpart. At the Hanford Site, uranium milling and uraniumore processing activities are not conducted. Therefore, the provisions of this subpart are not applicable to the Hanford Site.

\subsection{EMISSION OF RADON-220 FROM SOURCES CONTAINING URANIUM-232 AND THORIUM-232}

At the Hanford Site, atmospheric emissions of ${ }^{220} \mathrm{Rn}$ are calculated from ${ }^{212} \mathrm{~Pb}$ values measured in particulate samples collected from the 291-A-1 stack of the PUREX facility. Calculating ${ }^{220} \mathrm{Rn}$ emissions is required because ${ }^{232} \mathrm{Th}$ decays to ${ }^{220} \mathrm{Rn}$, which has a half-life of 55.6 seconds. Because ${ }^{220} \mathrm{Rn}$ is a gas, it passes unimpeded through the HEPA filters and sample filters in the PUREX stack. However, ${ }^{212} \mathrm{~Pb}$ is a ${ }^{220} \mathrm{Rn}$ decay product, has a half-life of 10.64 hours, and is a particle that can be captured on record sample filters. Sample filters are collected daily from the PUREX stack and analyzed on the Hanford Site within 5 hours from the time of collection. In 1992 , a calculated total of $34 \mathrm{Ci}$ of ${ }^{220} \mathrm{Rn}$ was emitted from the PUREX stack.

\subsection{SOURCES OF RADON-222}

Radon-222 is a decay product in the ${ }^{238} \mathrm{U}$ decay chain. The contribution of ${ }^{222} \mathrm{Rn}$ to the dose received by any member of the public can be calculated after removing the background uranium activity from soil analytical data. The radon flux that could be derived from any residual or above-background radioactivity, as a measure of the Hanford Site component, appears to be extremely small and would result in an offsite dose much smaller than $0.1 \mathrm{mrem} / \mathrm{yr}$ EDE.

Surface-soil samples were collected at locations both on and off the Hanford Site. The purpose of this sampling is to detect the possible buildup of radionuclides deposited by air emissions from Hanford Site facilities. Samples are collected at relatively undisturbed nonagricultural sites so that natural deposition and buildup processes would be represented. Because the radionuclides of interest were present in worldwide fallout or occurred both naturally and in Hanford Site emissions, these radionuclides were expected in all samples.

An assessment of radionuclide contributions from Hanford Site operations was made by comparing results from samples collected: (1) on the Hanford Site with those collected off the Hanford Site, (2) around the Hanford Site perimeter with those collected at distant locations, and (3) downwind (primarily east and south of the Hanford Site) with those collected from generally upwind and distant locations. Analytical results obtained from each 
location in 1991 were compared to results obtained from the same location in previous years. The comparison revealed no indication of trends or ircreases in the conceritrations of radionuclides in the offsite environment that could be attributed to Hanford Site activities. Because the data generally indicate that the onsite concentrations of these radionuclides are no higher than offsite concentrations, there should not be a net release of ${ }^{222} \mathrm{Rn}$ from the Hanford Site that would contribute to the offsite public dose.

\subsection{EMISSION POINTS SUBJECT TO MONITORING REQUIREMENTS OF 40 CFR 61.93(b) AND THE COSTS TO UPGRADE NONCOMPLIANT MONITORING SYSTEMS}

All Hanford Site facilities were evaluated for compliance with 40 CFR 61 (EPA 1992). As of 1992, 127 emission point sources were registered with the Washington State Department of Health. Of these 127 sources, 12 facility emission points were identified as having the potential to exceed the threshold limit of $0.1 \mathrm{mrem} / \mathrm{yr}$ EDE (40 CFR 61.93(b)(4)(i)). Westinghouse Hanford manages seven of these emission points, and PNL manages five. These 12 emission points, referred to as major stacks, either have been or are being evaluated for monitoring upgrades to comply with the requirements of 40 CFR 61. All 12 stacks (with each respective facility and managing DOE contractor) are listed below, by area. (EPA Region 10 has still not accepted a Westinghouse Hanford method for determining potential emissions from stacks it manages having multiple stages of HEPA filters, assuming the treatment controls have been removed. The final resolution may change the current list of 12 stacks.)

- 200 East Area

- 291-A-1 (PUREX; Westinghouse Hanford)

- 291-B-1 (B Plant; Westinghouse Hanford)

- 296-A-22 (242-A Evaporator; Westinghouse Hanford)

- 296-A-40 (241-AP Tank Farm; Westinghouse Hanford)

- 200 West Area

- 291-Z-1 (PFP; Westinghouse Hanford)

- 291-T-1 (T Plant; Westinghouse Hanford)

- 300 Area

- 340-NT-EX (340 Waste Handling; Westinghouse Hanford)

- EP-324-01-S (324 Building; PNL)

- EP-325-01-S (325 Building; PNL)

- EP-327-01-S (327 Building; PNL)

- EP-327-02-V (327 Decontamination Cell; PNL)

- EP-3720-01-S (3720 Building; PNL). 
Westinghouse Hanford compared the monitoring systems of the seven major stacks it manages to the continuous monitoring criteria of 40 CFR 61.93(b) (EPA 1992). The findings of the point-by-point comparisons were submitted to EPA Region 10 for review. Westinghouse Hanford is continuing engineering studies on modifications that potentially may be required to the monitoring systems.

In 1991, PNL began upgrading its monitoring systems of the five major stacks it operates; that work should be completed in 1994. Costs for individual system upgrades ranged from $\$ 20,000$ to $\$ 450,000$. Compared to the PNL-managed stacks, those managed by Westinghouse Hanford are larger, more complex, and have considerably greater contamination. Because of these factors, monitoring system upgrades for each of the seven stacks managed by Westinghouse Hanford are estimated to cost between approximately $\$ 200,000$ and $\$ 5$ million. The number of stack monitoring systems requiring upgrading possibly could increase, depending on the method EPA Region 10 approves for determining whether a stack is "major."

\subsection{PERIODIC CONFIRMATORY MEASUREMENT PLANS}

The Hanford Site air emission points with exhaust fans potentially discharging radionuclides to the environment have permanently installed sampling systems. Nearly all of these sampling systems are operated continuously, with samples removed at various intervals for radioanalysis. The most common interval is weekly, for gross alpha and gross beta analyses. For those stacks having radioactivity above background levels, sample filters typically are composited on a quarterly interval and then analyzed for specific radionuclides, such as ${ }^{90} \mathrm{Sr}$ ard ${ }^{239,240} \mathrm{Pu}$.

At least half of the 127 registered stacks on the Hanford Site are discharging air without any radioactivity (i.e., none that is detectable above background levels). Samples are collected from these stacks either weekly, biweekly, monthly, or quarterly and analyzed. The analysis results are reported annually and used in calculating the offsite dose for determining compliance with EPA and DOE standards.

\subsection{STATUS OF COMPLIANCE WITH QA PROGRAM CRITERIA DESCRIBED IN APPENDIX B, METHOD 114, 40 CFR 61}

In December 1991, a document describing the Westinghouse Hanford Quality Assurance program was published and submitted to EPA. The document, Quality Assurance Program Plan for Radionuclide Airborne Emissions Monitoring (WHC 1991b) contained appendices providing a point-by-point comparison of current monitoring systems for the major stacks with the recommendations of 40 CFR 61, Appendix B, Method 114, (EPA 1992). A few months later, a second document, Quality Assurance Project Plan for Radioactive Airborne Emissions Data Compilation and Reporting (WHC 1992), also was published and submitted to EPA. 
In May 1992, PNL implemented the Quality Assurance Plan for Facility Effluent Monitoring, Quality Assurance Plan No. F0-011. The QA program described by this plan is based on the following documentation:

- $\quad$ EPA QAMS-005/80, interim Guidelines for Preparing Quality Assurance Project Plans

- DOE 5700.6C, Quality Assurance

- DOE 5400.1, General Environmental Protection Program

- DOE/EH-0173T, Environmental Regulatory Guide for Radiological Effluent Monitoring and Environmental Surveillance

- $\quad$ applicable criteria of ASME NQA-1, Quality Assurance Requirements for Nuclear Facilities, as reflected in PNL's Quality Assurance Manual, PNL-MA-70, and associated implementing procedures. 
DOE/RL-93-36

This page intentionally left blank. 


\subsection{REFERENCES}

Beres, D. A., 1990, The Clean Air Act Assessment Package - 1988 (CAP-88). A Dose and Risk Assessment Methodology for Radionuclide Emissions to Air, Vols. 1-3, U.S. Environmental Protection Agency, Washington, D.C.

Bisping, L. E., and R. K. Woodruff, 1992, Hanford Site Environmental Data for Calendar Year 1991 - Surface and Columbia River, PNL-8149, Pacific Northwest Laboratory, Richland, Washington.

Brown, R. M., 1989, "Tritium in the Environment," presentation at the Marko Symposium on Radiation Protection: Past and Future, March 20-22, 1989, Chalk River, Ontario.

DOE, 1988, General Environmental Protection Program, DOE Order 5400.1, U.S. Department of Energy, Washington, D.C.

DOE, 1991, Environmental Regulatory Guide for Radiological Effluent Monitoring and Environmental Surveillance, DOE/EH-0173T, U.S. Department of Energy, Washington D.C.

DOE-RL, 1988, Hanford Site Dangerous Waste Part A Permit Application, DOE/RL-88-21, U.S. Department of Energy, Richland Operations Office, Richland, Washington.

DOE-RL, 1991, Calendar Year 1990 Air Emissions Report for the Hanford Site, DOE/RL-91-10, U.S. Department of Energy, Richland Field Office, Richland, Washington.

DOE-RL, 1992, Radionuclide Air Emissions Report for the Hanford Site, Calendar Year 1991, DOE/RL-92-30, U. S. Department of Energy, Richland Field Office, Richland, Washington.

EPA, 1989, "National Emission Standards for Hazardous Air Pollutants," Subpart H, Title 40, Code of Federal Regulations, Part 61, U.S. Environmental Protection Agency, Washington, D.C.

Leigh, C. D., B. M. Thompson, J. E. Campbell, D. E. Longsine, R. A. Kennedy, and B. A. Napier, 1992, User's Guide for GENII-S: A Code for Statistical and Deterministic Simulations of Radiation Doses to Huma's from Radionuclides in the Environment, SAND91-0561A, Sandia National Laboratories, Albuquerque, New Mexico.

Napier, B. A., R. A. Peloquin, D. L. Strenge, and J. V. Ramsdell, 1988, GENII - The Hanford Environmental Radiation Dosimetry Softwar? System, PNL-6584, Vols. 1-3, Pacific Northwest Laboratory, Richland, Washington. 


\subsection{REFERENCES (continued)}

Uranium Mill Tailings Radiation Control Act of 1978, Public Law 95-604, 42 USC 7901.

WHC, 1991a, Unit Dose Calculation Methods and Summary of Facility Effluent Monitoring Plan Determinations, WHC-EP-0498, Westinghouse Hanford Company, Richland, Washington.

WHC, 1991b, Quality Assurance Program Plan for Radionuclide Airborne Emissions Monitoring, WHC-EP-0536, Westinghouse Hanford Company, Richland, Washington.

WHC, 1992, Quality Assurance Project Plan for Radioactive Airborne Emissions Data Compilation and Reporting, WHC-EP-0528, Westinghouse Hanford Company, Richland, Washington.

Woodruff, R. K., and R. W. Hanf, 1991, Hanford Site Environmental Report for Calendar Year 1991, PNL-8148, Pacific Northwest Laboratory, Richland, Washington. 


\section{DISTRIBUTION}

Number of copies

\section{OFFSITE}

4

U.S. Environmental Protection Agency, Region 10 1200 Sixth Avenue

Seattle, WA 98081

G. M. Leitch

AT-081

F. J. McCormick

AT-081

G. O'Neal

AT-081

R. W. Poeten

U.S. Environmental Protection Agency, Region 10

712 Swift Boulevard, Suite 5

Richland, V: A 99352

P. T. Day

2

Washington State Department of Health

Olympia, WA 98504-0095
A. W. Conklin
X. Fox-Williams

Washington State Department of Ecology

Olympia, WA 98504-8711

D. B. Jansen

Wishington Public Power Supply System

P.O. Box 968

Richland, Washington 99352

C. J. Card

1025

T. E. Chapman

1020

4 U.S. Department of Energy-Headquarters

R. F. Pelletier (3)

EH-232

M. K. Harmon

EM-442 
DOE/RL-93-36

DISTRIBUTION (continued)

Number of copies

QFFSITE

12

U.S. Department of Energy. Richland Operations Office

J. D. Bauer (4)

A5-15

S. S. Clark

R3-81

M. J. Furman

R3-80

J. E. Mecca (3)

R3-81

J. E. Rasmussen

A5-15

S. D. Stites

A5-15

DOE-RL Public Reading Room

$\mathrm{H} 2-53$

ONSITE

$14 \quad$ Pacific Northwest Laboratory

$\begin{array}{ll}\text { T. D. Chikalla } & \text { P7-75 }\end{array}$

E. G. Damberg P7-64

R. W. Hanf, Jr. K6-13

G. R. Hoenes $\quad$ P7-78

A. S. Ikenberry $\quad$ P7-64

S. J. Jette

P7-78

W. E. Kennedy, Jr. K3-54

$\begin{array}{ll}\text { D. L. Klages } & \text { P7-68 }\end{array}$

$\begin{array}{ll}\text { K. Rhoads } & \text { P3-54 }\end{array}$

J. K. Soldat K3-54

$\begin{array}{ll}\text { M. J. Sula } & \text { P7-68 }\end{array}$

H. T. Tilden II $\quad$ P7-68

R. K. Woodruff K6-13

Technical Files $\quad$ K1-11

179 Westinghouse Hanford Company

L. L. L. Adams T7-20

D. Alison T5-54

J. M. Barnett $\quad$ T1-30

D. L. Barron $\quad$ S2-62

J. A. Bates H6-22

M. L. Bell T6-16

L. D. Berneski L4-96 


\section{DISTRIBUTION (continued)}

Number of copies

QNSITE

Westinghouse Hanford Company (continued)

ii.. J. Bliss

B3-04

R. E. Bolls

N3-13

R. J. Boom

T1-30

W. W. Bowen

S6-65

M. J. Brown

H6-20

S. A. Burris

T1-30

G. D. Carpenter

H6-30

G. J. Carter, Jr.

T1-06

K. A. Coffman

A2-29

J. M. Colby

H5-07

S. N. Cory

H6-20

J. E. Cottrell

T7-20

P. J. Crane

H4-14

G. M. Crummel

R1-51

B. L. Curn

$\mathrm{X} 0-21$

W. E. Davis

H6-70

H. L. Debban

$\mathrm{X} 0-43$

G. V. Delisle

L7-05

L. P. Diediker (75)

T1-30

D. A. Dodd

T6-50

J. J. Dorian

H6-30

D. S. Duff

L6-39

B. B. Emory

B. G. Erlandson

X3-70

H6-20

D. G. Farwick

H4-16

G. W. Faulk

T3-28

T. P. Frazier

T1-30

J. C. Fulton

R3-56

K. A. Gano

$\mathrm{X} 0-21$

R. G. Gant

$\mathrm{X} 0-41$

C. J. Geier

H6-21

E. M. Greager

H6-30

W. J. Gretsinger

R4-01

M. Haas

T5-50

K. A. Hadley

N1-35

M. J. Hall

T6-12

W. H. Hamilton, Jr.

N3-10

D. W. Hendrickson

R4-03 


\section{DISTRIBUTION (continued)}

\section{Number of copies}

ONSITE Westinghouse Hanford Company (continued)

D. R. Herman

G. W. Jackson

D. B. Jensen

S4-01

A. R. Johnson

H6-21

H6-30

R. E. Johnson

H6-30

J. R. Kasper

T1-30

R. J. Landon

R2-50

G. L. Law ;

H6-22

G. J. LeBaron

H6-25

R. E. Lerch

S6-19

J. J. Luke

B3-63

R. P. Marshall, Jr.

H6-25

T6-14

P. J. Martell

T1-30

D. J. McBride

T5-54

H. E. McGuire

B3-63

S. M. McKinney

T1-30

J. C. Midgett

S6-15

P. C. Miller

N2-04

M. R. Morton

R2-77

K. J. Moss

R3-12

R. R. Nielson

T6-12

D. J. Newland

R2-28

J. M. Nickels

H6-22

J. K. Perry

H6-20

R. D. Pierce

T5-30

L. L. Powers

L4-96

R. K. P'Pool

T1-30

R. G. Ranade

B2-16

D. E. Rasmussen

L4-30

J. M. Ring

H6-30

J. A. Rivera

B2-16

W. G. Ruff

R3-50

J. P. Schmidt

$\mathrm{X} 0-41$

J. W. Schmidt

H6-30

M. J. Silvia

H6-25

C. M. Smith

H6-30

D. R. Speer

R1-48

D. J. Swaim

B3-51 


\section{DISTRIBUTION (continued)}

Number of copies

ONSITE Westinghouse Hanford Company (continued)

R. W. Szelmeczka T4-06

S. P. Thomas T1-30

R. J. Thompson S6-17

R. R. Thompson L4-96

M. E. Thurman T3-28

A. L. Trego B4-52

G. E. VanSickle H6-20

H. R. Vogel S6-61

E. C. Vogt $\quad$ T5-50

D. J. Watson X0-41

R. D. Weissenfels $\quad$ S6-70

R. D. Wojtasek H6-27

C. D. Wollam S6-19

Central Files $\quad$ L8-04

Correspondence Control A3-01

Document Processing and Distribution (2) L8-15

EDMC $\quad$ H4-22

Information Release Administration (3) R1-08

$\begin{array}{ll}\text { Publications Services } & \text { A2-25 }\end{array}$ 
DOE/RL-93-36

This page intentionally left blank. 

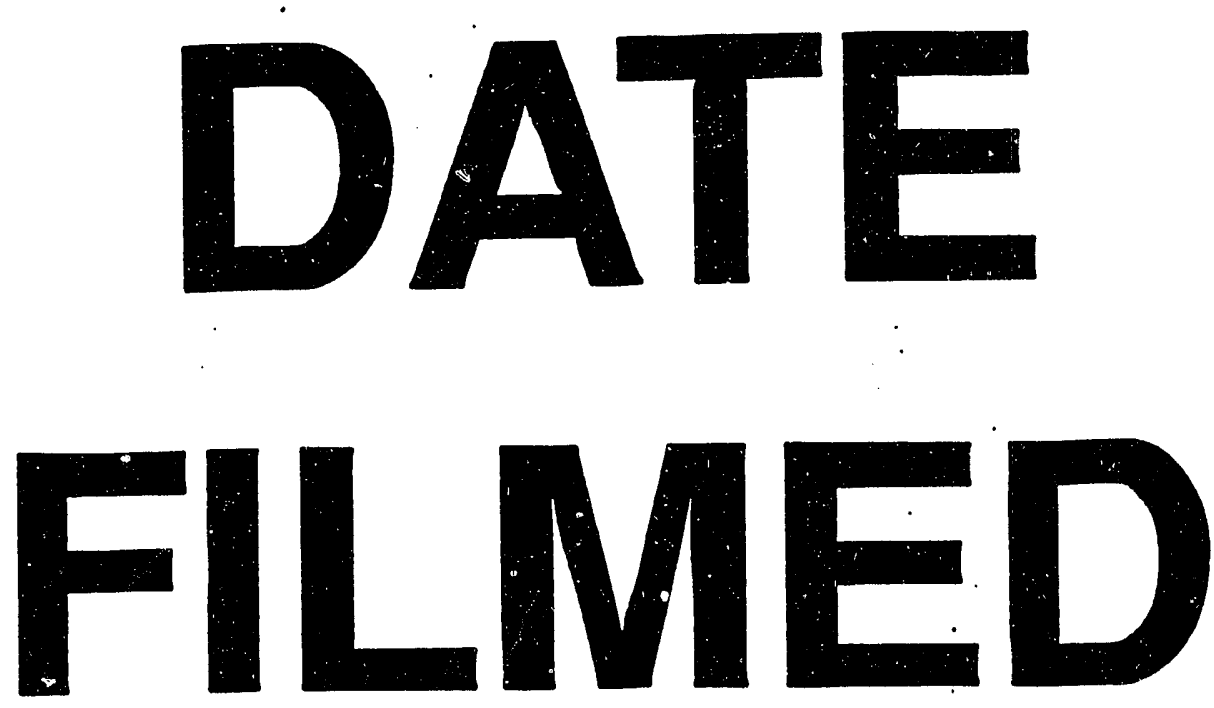

$10 / / 4 / 93$
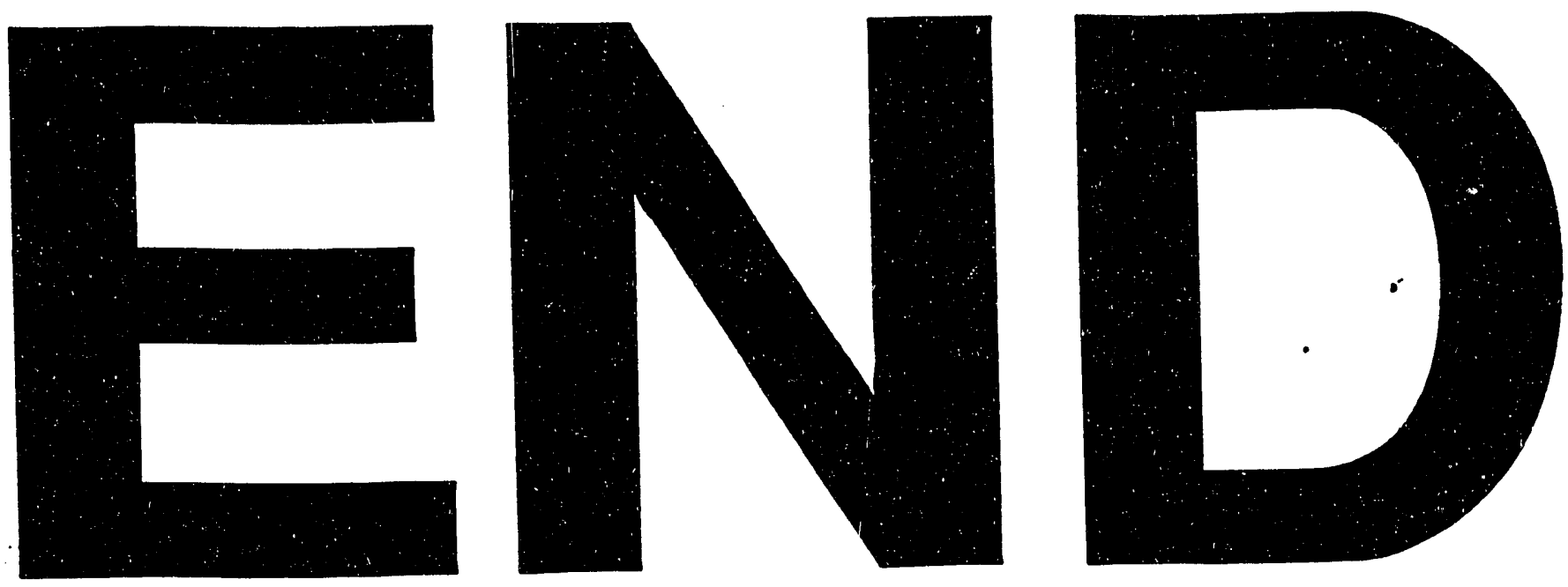
